
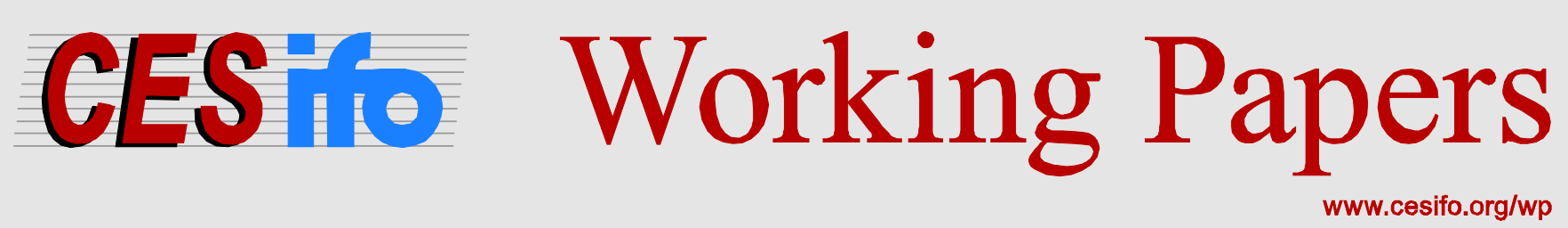

\title{
Market Size and TFP in New New Trade Theory
}

\author{
Gabriel Felbermayr \\ Benjamin Jung
}

CESIFO WORKING PAPER NO. 5583

CATEgory 8: TRAde Policy

OCTOBER 2015

An electronic version of the paper may be downloaded

- from the SSRN website:

- from the RePEc website:

- from the CESifo website:

wWw.SSRN.com

www.RePEc.org

www.CESifo-group.org/wp 


\title{
Market Size and TFP in New New Trade Theory
}

\begin{abstract}
Recent trade theory in the Krugman (1980) tradition predicts that countries with larger market size enjoy higher levels of total factor productivity (TFP) - and equivalently of real per capita income or welfare - as a smaller fraction of spending on inputs is affected by trade costs. However, in cross-country data, there is no such positive correlation between market size and TFP. We argue that models with heterogeneous firms and selection help to reconcile theory and data. While they do feature a home market effect - larger countries have an over-proportionate share of firms - and, therefore, have more input varieties available, the average productivity of firms is lower as greater market size protects inefficient firms. To reconcile theory with data, we show that a lower degree of external economies of scale (EoS) is needed than what is implicitly assumed in the usual formulation of aggregate CES production functions. Whether trade liberalization triggers convergence or divergence of TFP also depends on the strength of EoS.
\end{abstract}

JEL-Codes: F120, R120.

Keywords: trade, monopolistic competition, heterogeneous firms, total factor productivity, scale effects, home market effect.

Gabriel Felbermayr

Ifo Institute - Leibniz Institute for

Economic Research

at the University of Munich

Poschingerstrasse 5

Germany-81679 Munich

felbermayr@ifo.de

October 16, 2015

We are grateful to Costas Arkolakis,Daniel Bernhofen, Johannes Bröcker, Jonathan Eaton, Carsten Eckel, Peter Egger, Sebastian Krautheim, Udo Kreickemeier, Mario Larch, and Horst Raff for discussion and suggestions, as well as to seminar participants at the Universities of Bielefeld, Kiel, Mainz, Munich, Nottingham, Passau, and Tübingen for comments. 


\section{Motivation}

Policy makers and the public are concerned with the relative size of their economies. This is most clearly visible in the discussion about the increase of the relative weight of emerging countries. $^{1}$

New Trade Theory (Krugman,1980; Helpman and Krugman, 1985) with homogeneous firms provides a theoretical rationale for this optimistic view of scale. With trade costs, product differentiation, increasing returns to scale at the firm-level, and imperfect competition, firms prefer to locate in the larger country, ceteris paribus, since this allows the majority of sales to be carried out without incurring transportation costs. This home market effect (HME) leads to lower prices, therefore benefiting consumers. In the standard treatment with a conventional Dixit-Stiglitz CES aggregate production or utility function, large closed economies also benefit from external economies of scale as they tend to have a larger number of varieties of goods and inputs available. With trade, they benefit from larger global scale.

However, there is very little support for a positive correlation between total factor productivity (TFP) or welfare and scale as measured by population size or employment. We use new theory-consistent measures of TFP available from Feenstra et al. (2015) to document this robust findings. Scale is never positively correlated to TFP. If anything, the opposite is the case, in particular, after controlling for time-invariant country characteristics. ${ }^{2}$

In a closed economy models without selection induced by fixed market access costs and productivity dispersion amongst producers, one can shut down the positive correlation between TFP and size simply by neutralizing external economies of scale (EoS) as in Dixit and Stiglitz (1977) or in Benassy (1998). However, in open economies, this is not sufficient as all varieties are available in all countries and the larger country has the lower price index, regardless of the extent of external EoS. However, in the presence of a selection effect as in Melitz

\footnotetext{
${ }^{1}$ In a special report, The Economist (Sept 24, 2011) argues "The shift in economic power from West to East is accelerating ... The rich world will lose some of its privileges”. It provides examples of policy makers' obsession with "grandeur and decline" and China "Becoming number one".

${ }^{2}$ So far, there is little empirical work on the effect of country size on macroeconomic outcomes. Rose (2006) is an early exception. Also note that, starting with Jones (1995), the growth literature has discussed the empirical link between scale and growth rates or income levels, which led to the development of semi endogenous and scale-free growth models. The trade literature is only starting to address the issue; see below.
} 
(2003), the average productivity of firms is smaller in the larger economy, and this counteracts the positive effects of scale just mentioned. ${ }^{3}$ Moreover, the extent of external EoS depend on scale as the availability of varieties is greater in larger countries. Hence, models with selection effects are better suited to fit the empirical evidence than the simple Krugman (1980) model.

We make these claims with the help of an asymmetric two-country single-sector Melitz (2003) model where firm productivities are Pareto distributed and the aggregate CES production function allows for a flexible degree of external economies of scale. While the symmetric case is easy to handle even with general distribution functions, the asymmetric model has been solved analytically only in a small open economy setup (Demidova and RodriguezClare, 2013) or under the assumption of a linear outside sector which pins down wages (Demidova, 2008). ${ }^{4}$ We provide an intuitive graphical device to solve the asymmetric two-country case that should be helpful in other applications as well.

A key element in the analysis is the so called home market effect which has not been studied in single-sector models with selection effects à la Melitz (2003). We show that, in the presence of trade costs, in the larger country the selection effect of international trade is weaker as higher market size protects inefficient domestic firms. Moreover, the larger country hosts an over-proportional share of firms. While this finding is similar to the prediction of Helpman and Krugman (1985), the mechanisms are different. In multi-sector models, the result is generated by endogenous labor supply to the differentiated good sector. In the single-sector Melitz (2003) model with fixed labor supply, the result is generated by selection. In the larger country, a larger fraction of firms remains purely domestic and therefore relatively smaller. This allows a larger number of them to exist in equilibrium, which, in turn, increases welfare per capita in the larger country due to the availability of a larger range of varieties. Importantly, while the mass of firms attempting entry is strictly proportional to the endowment size, the likelihood of a given firm to successfully cover its fixed costs is greater in the larger market.

\footnotetext{
${ }^{3}$ Selection arises from the interplay between fixed market access costs and productivity heterogeneity. There is ample empirical evidence for the importance of heterogeneity and fixed costs, as well as for the resulting selection (for recent surveys see Melitz and Trefler (2012) and Melitz and Redding (2014).

${ }^{4}$ Demidova and Rodriguez-Clare (2013) show that eliminating the assumption of an outside sector reverses the welfare effects of unilateral trade liberalization in Melitz and Ottaviano (2008) or Demidova (2008).
} 
Symmetric trade liberalization magnifies the effect of country size on concentration, which is similar to the standard Home Market Magnification (HMME). ${ }^{5}$ Moreover, concentration of production in the larger country is increasing in the degree of productivity dispersion, which constitutes a further HMME.

When a country commands a larger share of world population, its attractiveness as a production location increases. The increased demand for labor is accommodated along two margins: first, the relative wage of the country goes up; second, the average size of firms goes down. In a model with exogenous wages, the first channel would not be present, and the HME is of maximum size. In a model with homogeneous firms, firm size cannot adjust, and the wage needs to rise until the number of firms is exactly proportional to the labor force. The more dispersed the productivity distribution, the stronger the link between average firm size and the number of firms, and, accordingly, the more pronounced is the HME.

The larger country not only has a larger mass of firms, it also has access to an overproportional mass of inputs. Thus, if there are external EoS, the larger country has higher TFP. However, it also has the lower average productivity. If that selection channel is strong enough relative to the effects of external EoS and of the HME, the model can capture the low correlation between scale and TFP measured in the data. This requires, however, that the degree of external EoS is lower than the one implicit in the standard formulation of the CES aggregate in the Krugman (1980) or Melitz (2003) models.

Whether trade liberalization results in convergence of TFP (and, thus, of real per capita income) also depends on the strength of external EoS. In the standard CES configuration, it leads to convergence, but if the degree of EoS is weaker than what is needed to generate a non-decreasing effect of scale on TFP, trade liberalization generates divergence.

Traditionally, the role of country scale in models of open economies has been discussed in the context of the HME. It is a unique feature of new trade theory models à la Krugman (1980). Therefore, providing statistical proof for its existence amounts to confirming the validity of

\footnotetext{
${ }^{5}$ In a single-sector Melitz (2003) model, symmetric trade liberalization makes both countries more attractive in terms of export opportunities, but favors the smaller, more open country. Free entry then requires that the entry probability falls by more in the smaller country, resulting in relatively weaker selection in the large country and, in turn, relatively more active firms.
} 
new trade theory. Indeed, the HME enjoys some empirical support. ${ }^{6}$ However, with very few exceptions (see below), the empirical literature has not tested for the positive scale-welfare (or TFP) link that is implicit in the new trade models. In the growth literature, Jones' (1995) empirical finding has triggered the development of scale-free models of endogenous growth. The trade literature is only starting in this direction. Ramondo et al. (2015) present a recent exception and argue that the scale effect is counterfactual. They use a Ricardian trade model (with heterogeneous firms and no HME), where the state of technology depends on scale, and argue that traditional models exaggerate the role of country scale by neglecting domestic trade costs. We address the same empirical puzzle, but our model still features a HME and the proposed mechanism relies on firm heterogeneity and selection.

Our work also addresses the common assumption of a linear outside sector featuring perfect competition and a freely tradeable good. ${ }^{7}$ Crucially for our paper, this assumption would imply that the sorting of firms into markets depends only on trade costs but not on relative country size, so that there is no selection effect based on scale. In a model with an outside sector and endogenous mark-ups, as in Melitz and Ottaviano (2008), the larger country exhibits lower mark-ups, lower prices, and higher average productivity, further strengthening the positive link between scale and welfare. In contrast, in the single sector model with endogenous wages, firms in the larger country are on average less productive and charge higher prices.

The remainder of the paper is structured as follows. Chapter 2 describes the empirical link between TFP and market size. Chapter 3 describes the model. Chapter 4 develops a simple graphical tool to solve the model and proves the existence of a home market effect in a singlesector model with selection and endogenous wages. Chapter 5 analyzes the implications of the home market effect for TFP for a generalized aggregate CES production function. Chapter 6 concludes. Technical details are relegated to the Appendix.

\footnotetext{
${ }^{6}$ See, e.g., Davis and Weinstein (1999, 2003), Feenstra et al. (2001), Head and Ries (2001), or Hanson and Xiang (2004). Theoretically, the HME has been criticized for not being robust; see Davis (1998).

${ }^{7} \mathrm{~A}$ number of papers has used a linear, freely traded, perfectly competitive outside sector in a Melitz-type environment for the sake of analytical tractability; see Helpman et al. (2004), Grossman et al. (2006), Chor (2009), Baldwin and Okubo (2009), Baldwin and Forslid (2010), and Ossa (2011).
} 


\section{The Scale-TFP Link in the Data}

Do larger countries enjoy higher levels of per capita income or of TFP? Economic theory does not provide clear-cut answers. Neoclassical growth theory predicts neutrality. Ricardian or Armingtonian trade models associate larger size with lower terms of trade and, thus, lower per capita incomes. Early endogenous growth models (e.g., Romer, 1990) and trade models featuring featuring trade costs and free entry (such as Krugman, 1980) produce a positive link between size and TFP (per capita income); the former because larger markets induce more innovation, the latter because in larger markets less resources are wasted on trade costs. The data, however, tell a different story. Using newly available theory-consistent TFP measures for a large sample of countries, we show that scale is either irrelevant or even harmful.

In the empirical literature on the determinants of per capita income, results on scale effects arise as mere byproducts in regressions that study the effects of trade openness, geography, or institutions. In the instrumental variables (IV) studies of Frankel and Romer (1999) and Alcalá and Ciccone (2004) population size is positively related to GDP per capita and TFP, respectively. However, Irwin and Terviö (2002) use the same IV strategy to mostly find negative effects. Moving from cross-sections to a panel framework to control for unobserved heterogeneity, Felbermayr and Gröschl (2013) report negative scale effects. To our knowledge, Rose (2006) is the only paper that explicitly searches for scale effects in repeated crosssections of countries. He finds negative effects that are particularly pronounced in fixedeffects models. ${ }^{8}$

These findings are interesting, but of only limited use for our purposes. The reason is that GDP per capita is not the appropriate measure of welfare in a Krugman (1980) or Melitz (2003) model. Luckily, the new Penn World Tables 8.1 (Feenstra et al., 2015) implement the theory-consistent TFP measure of Basu et al. (2012), which is a measure of welfare in the single-factor framework that we use below.

In Figure 1, we plot coefficients obtained by regressing the log of TFP against the log of

\footnotetext{
${ }^{8}$ Using time-series techniques, Jones (1995) finds no empirical evidence for scale effects in growth setups. This has led to the development of appropriate modifications of theoretical models.
} 
Figure 1: Scale and TFP across countries: elasticity estimates in cross-sections
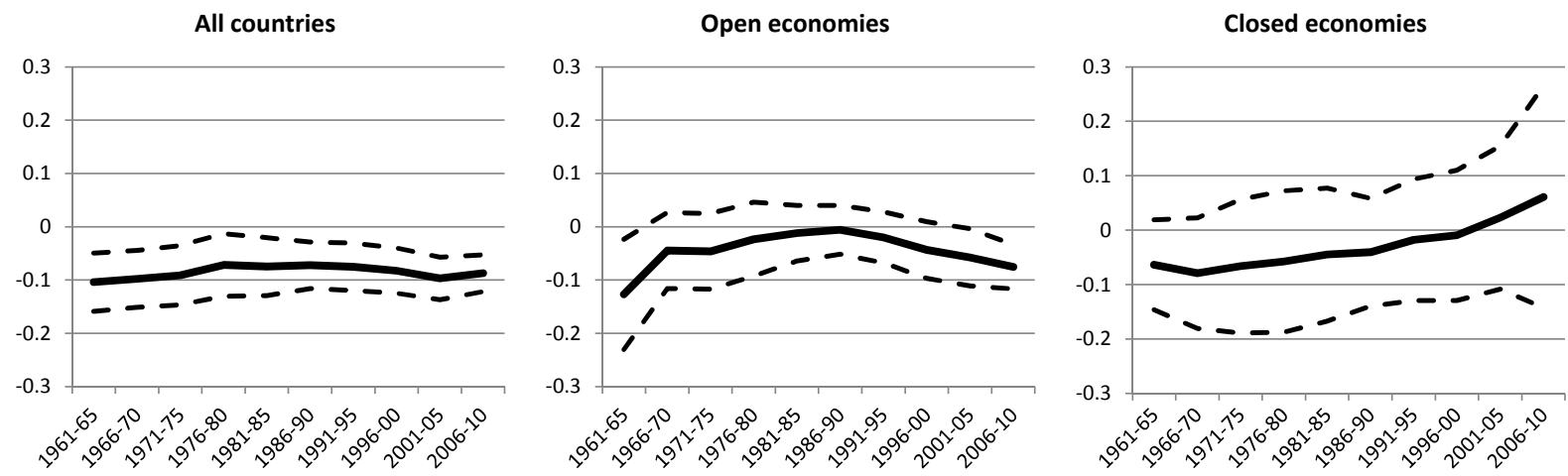

Notes: Data from Penn World Tables 8.1 (Feenstra et al., 2015); series cwtfp (welfare-relevant TFP levels at current PPPs) and pop, both relative to the respective US values; balanced sample $(N=68)$. Closedness defined as openness (PWT 8 series csh_x-csh_m) lower than 30\% Figures show coefficients obtained from regressing the log of of cwtfp on the log of pop. 95\% confidence intervals based on robust standard errors plotted.

population (expressing all variables relative to the US) in cross-sections containing averaged data for five year windows. Fixing a sample of 68 countries for which we have the TFP measure for all 10 periods, we find a scale elasticity of TFP of about -0.1 ; see the left-hand diagram in Figure 1. Focusing on the subsamples of open economies (middle diagram), the picture becomes more blurred, but estimates remain negative. ${ }^{9}$ The overall picture from cross-sections of countries is that - at best - scale is unrelated to TFP.

The results in Figure 1 may be biased due to unobserved heterogeneity. ${ }^{10}$ Indeed, firstdifferenced panel regressions confirm this suspicion. Table 1 shows negative scale elasticities that remain after controlling for openness but disappear in a sample of 'closed' economies. ${ }^{11}$

In this paper, we argue that this stylized fact - the absence of a positive scale effect in cross-sections of countries - is compatible with the existence of a home market effect and external economies of scale if country size is allowed to affect the composition of the firm population.

\footnotetext{
${ }^{9}$ In Table 2 in Appendix A we show regressions with additional controls (the log of area as one more scale variable and a dummy for landlockedness as an exogenous proxy for openness) for recent periods.

${ }^{10}$ A country's geographical, climatic, or cultural circumstances may at the same time result in lower TFP and lower population size; thereby biasing the scale elasticity reported in Figure 1 towards zero.

${ }^{11}$ Table 3 in Appendix A shows that the finding of a negative elasticity is very robust: it holds even with alternative proxies of welfare, for other measures of scale (employment), in both fixed-effects and first-differenced models, and in balanced and unbalanced samples. Only in 2 out of 96 regressions do we find positive coefficients; none is statistically significant.
} 
Table 1: Scale and TFP in panel regressions (first-differenced model)

\begin{tabular}{lcccccc}
\hline \hline \multirow{2}{*}{ SAMPLE } & $\begin{array}{c}(1) \\
\text { unbalanced }\end{array}$ & $\begin{array}{c}(2) \\
\text { balanced }\end{array}$ & $\begin{array}{c}(3) \\
\text { unbalanced }\end{array}$ & $\begin{array}{c}(4) \\
\text { balanced }\end{array}$ & $\begin{array}{c}(5) \\
\text { closed }\end{array}$ & $\begin{array}{c}(6) \\
\text { open }\end{array}$ \\
\hline \multirow{2}{*}{ ln population rel. to US } & $-0.320^{* * *}$ & $-0.345^{* * *}$ & $-0.332^{* * *}$ & $-0.353^{* * *}$ & -0.0288 & $-0.387^{* * *}$ \\
& $(0.0756)$ & $(0.116)$ & $(0.0808)$ & $(0.115)$ & $(0.247)$ & $(0.0802)$ \\
Openness & & & -0.0192 & -0.0145 & & \\
& & & $(0.0317)$ & $(0.0209)$ & & \\
\hline Observations & 815 & 612 & 815 & 612 & 294 & 521 \\
(within) R-squared & 0.236 & 0.226 & 0.237 & 0.226 & 0.247 & 0.264 \\
Number of countries & 110 & 68 & 110 & 68 & 61 & 96 \\
\hline \hline
\end{tabular}

Notes: All regressions include a full set of period dummies. Robust standard errors (in parentheses) control for clustering at the country level: $* * * p<0.01, * * p<0.05, * p<0.1$. See Figure 1 on data sources.

\section{The Model}

\subsection{Basic environment}

The model is the basic extension of Melitz (2003) to the case of two large asymmetric countries, indexed by $i \in\{H, F\}$. Each country is populated with $L_{i}$ identical households. Labor is the only factor of production. ${ }^{12}$ Importantly, each household inelastically supplies one unit of labor. We denote wages by $w_{i}$. The representative consumer achieves utility from consuming a homogeneous final good.

Following Benassy (1998) and Blanchard and Giavazzi (2003), the final output is produced according to a generalized CES aggregate of all available intermediate goods:

$$
Y_{i}=\left[N_{i}^{\frac{\mu-1}{\sigma}} \int_{z \in \Omega_{i}} q_{i}[z]^{\frac{\sigma-1}{\sigma}}\right]^{\frac{\sigma}{\sigma-1}}
$$

where $q_{i}[z]$ is input of variety $z, \sigma>1$ is the constant elasticity of substitution between any two varieties, $\Omega_{i}$ is the set of available intermediate inputs, and $N_{i}$ is a measure of this set. Due to the presence of selection effects, $N_{i}$ is a non-trivial endogenous variable. ${ }^{13}$ The parameter $\mu$ controls the degree of external economies of scale. In order to understand the

\footnotetext{
${ }^{12}$ We devise the model as a single-factor framework. Multiple (non-traded) factors can be easily accommodated if one is willing to assume that variable and fixed inputs are in terms of a composite input, which combines different factor services in a constant returns to scale fashion. That composite input takes the role of labor in our analysis; all results stated in this paper would continue to hold.

${ }^{13}$ In a single-sector Krugman (1980) model, $N_{i}$ would be proportional to world endowments and independent of trade costs.
} 
role of $\mu$, consider the hypothetical case where all available inputs are entirely homogeneous and enter symmetrically into the final good. Then, the aggregate production function reads $Y_{i}=N_{i}^{\frac{\mu}{\sigma-1}} \times N_{i} q_{i}$, where the first term is TFP and the second is a trivial output index. Later in this paper we will show how these elements depend on scale when firms are heterogeneous, and trade in inputs is subject to variable and fixed foreign market access costs. Since firms do not internalize the effect of their entry decisions on $N_{i}$, equation (1) exhibits external economies of scale for $\mu>0$ and diseconomies of scale for $\mu<0$. For $\mu=0$, (dis)economies of scale are muted. ${ }^{14}$ Ardelean (2007) argues that $\mu \in(0,1)$ is the empirical relevant case. So, everything else equal, TFP is higher the larger the mass of available input varieties. In a reduced form equation (1) captures the idea, that a higher mass of varieties makes it more likely that idiosyncratically different final output producers find inputs that better suit them, which improves productivity.

Optimal demand for input variety $z$ is given by $q_{i}[z]=E_{i} P_{i}^{\sigma-1} N_{i}^{\mu-1} p[z]^{-\sigma}$, where $p[z]$ is the price of input variety $z, E_{i}$ is aggregate expenditure, and $P_{i}$ is the price index of the final good given by

$$
P_{i}=\left[N_{i}^{\mu-1} \int_{z \in \Omega_{i}} p[z]^{1-\sigma}\right]^{-\frac{1}{\sigma-1}} .
$$

Producers of intermediate inputs compete monopolistically in a single sector. After paying fixed setup costs $w_{i} f^{e}$, they obtain information about their productivity level $\varphi$. Productivities are sampled from a Pareto distribution whose c.d.f. is given by $G_{i}[\varphi]=1-\varphi^{-\beta}$, as in standard quantitative trade literature. ${ }^{15}$ The shape parameter $\beta$ is inversely related to productivity dispersion. Output is linear in $\varphi$. A firm in country $i$ pays fixed market access costs $w_{i} f_{i j}$ to serve consumers in country $j$. Selection implies that a firm does not necessarily serve one or both markets. Whenever advantageous, we use $f_{i j}=f_{j i}=f^{x}$ and $f_{i i}=f_{j j}=f^{d}$. As usual, exporting involves symmetric iceberg trade costs $\tau_{i j}=\tau_{j i}=\tau \geq 1$, where $\tau_{i i}=1$. Then, $\tau_{i j} w_{i} / \varphi$ is the marginal cost of producing one unit of output in $i$ and selling to $j$. In the

\footnotetext{
${ }^{14}$ The generalized CES aggregate (1) is introduced in the working paper version of Dixit and Stiglitz (1977). Many macroeconomic applications rule out economies of scale by setting $\mu=0$; see, e.g., Blanchard and Giavazzi (2003). One can also interpret equation (1) as a generalized CES utility function.

${ }^{15}$ Note that each variety $z$ is produced by a single firm with productivity level $\varphi$. We henceforth index varieties by $\varphi$. With the CES aggregate and Pareto, trade flows obey gravity; see Costinot and Rodriguez-Clare (2015).
} 
following description of equilibrium conditions, we will be very brief since the model is very standard.

\subsection{Equilibrium conditions}

The first set of equilibrium conditions are zero cutoff profit conditions. They pin down the minimum productivity level $\varphi_{i j}^{*}$ required for a firm in country $i$ to make at least zero profits by serving the final good producer in country $j$. Since we have two countries, there are four of those conditions:

$$
\frac{E_{j} N_{j}^{\mu-1}}{\sigma}\left(\frac{\rho P_{j}}{\tau_{i j} w_{i}} \varphi_{i j}^{*}\right)^{\sigma-1}=w_{i} f_{i j}, \quad i \in\{H, F\}, j \in\{H, F\},
$$

where $\rho=(\sigma-1) / \sigma \in(0,1)$ is the inverse of the mark-up. ${ }^{16}$ Since we assume balanced trade, aggregate expenditure $E_{j}$ is equal to national income $w_{j} L_{j}$. The price index is given by

$$
P_{i}^{1-\sigma}=\theta N_{i}^{\mu-1} \sum_{j \in\{H, F\}} M_{j i}\left(\frac{\rho \varphi_{j i}^{*}}{\tau_{j i} w_{j}}\right)^{\sigma-1}, i \in\{H, F\}
$$

where $M_{j i} \equiv \varphi_{j i}^{*} M_{j}^{e}$ denotes the (endogenous) mass of inputs produced in $j$ and available in $i$, and $M_{i}^{e}$ is the mass of entrants in country $i . \theta \equiv \beta /(\beta-(\sigma-1))$ is a strictly positive constant. ${ }^{17}$ The mass of available intermediate inputs is given by $N_{i}=M_{i i}+M_{j i}$. The left hand side of (2) denotes profits of a firm with labor productivity $\varphi_{i j}^{*}$. They are proportional to aggregate profits $E_{j} / \sigma$. The right hand side denotes the value of fixed market entry costs. Firm-level profits increase in the foreign price level as the firm's competitive position there is improved; they decrease in $w_{i}$ for the opposite reason. Hence, for a given demand level $E_{j} P_{j}^{\sigma-1} N_{j}^{\mu-1}$, an increase in the wage rate $w_{i}$ must be accompanied by stronger selection (an increase in the entry cutoff $\varphi_{i j}^{*}$ ) in order to restore zero profits of the cutoff firm. For a given cost situation in country $i$ (wage $w_{i}$ ), also less productive firms can successfully enter country $j$ if a rise in population (rise in $L_{j}$ ) increases demand.

\footnotetext{
${ }^{16}$ Empirical evidence suggests that only the most productive firms export. The model reproduces this stylized fact if parameters are such that $\tau_{i j}^{\sigma-1} \frac{f_{i j}}{f_{i i}} \frac{A_{j}}{A_{i}}>1$, where $A_{i} \equiv E_{i} P_{i}^{\sigma-1} N_{i}^{\mu-1}$ denotes the (endogenous) demand level. We relegate the analysis of the case where this condition is violated to Appendix D.2.

${ }^{17}$ This is to ensure that the variance of the size distribution is finite.
} 
In the analysis below, we are only interested in the attractiveness of a certain market $j$ for foreign relative to domestic firms. Using the import and the domestic zero cutoff profit conditions (2), we obtain

$$
\left(\frac{\varphi_{i j}^{*}}{\varphi_{j j}^{*}}\right)^{\sigma-1}=\tau_{i j}^{\sigma-1} \frac{f_{i j}}{f_{j j}}\left(\frac{w_{i}}{w_{j}}\right)^{\sigma} .
$$

By taking the ratio of the two zero cutoff profit conditions, we eliminate the aggregate demand $E_{j} P_{j}^{\sigma-1} N_{j}^{\mu-1}$. Thus, for a given relative wage, the relative entry cutoff does not depend on external economies to scale $\mu$. Intuitively, it is increasing in variable trade $\operatorname{costs} \tau_{i j}$, relative market entry costs $f_{i j} / f_{j j}$, and relative production costs $w_{i} / w_{j}$.

The second set of equilibrium conditions are free entry conditions. In each country, firms invest fixed setup costs until expected profits from entering $(\theta-1) w_{i} \sum_{j} m_{i j} f_{i j}$ are equal to entry costs discounted by the probability of successful entry $p_{i}^{i n}=1-G\left[\varphi_{i i}^{*}\right]$ for $i \in\{H, F\}, j \in$ $\{H, F\}$. The $t w o$ free entry conditions therefore are

$$
(\theta-1) p_{i}^{i n} \sum_{j \in\{H, F\}} m_{i j} f_{i j}=f^{e}, i \in\{H, F\}
$$

where $m_{i j}=\left(1-G\left[\varphi_{i j}^{*}\right]\right) /\left(1-G\left[\varphi_{i i}^{*}\right]\right)$ is the fraction of firms located in country $i$ which serve market $j$. Note that wages have dropped out from this condition. They nevertheless affect entry in general equilibrium. For a given probability of successful entry $p_{i}^{i n}$ and a given demand level $E_{j} P_{j}^{\sigma-1} N_{j}^{\mu-1}$, an increase in $w_{i}$ comes along with decline in the export participation rate $m_{i j}$, which implies a fall in expected profits. Hence, an increase in the wage $w_{i}$ must be accompanied by an increase in the probability of successful entry in order to restore zero profits in expectations.

Moreover, the free entry condition (5) does not depend on external economies of scale $\mu$. The reason is that variable profits of a firm with productivity $\varphi$ can be written as a function its revenue. Firm revenue, in turn, can be expressed as $r_{i j}[\varphi]=\left(\frac{\varphi}{\varphi_{i j}^{*}}\right)^{\sigma-1} r_{i j}\left[\varphi_{i j}^{*}\right]$, where revenue of the cutoff firm is pinned down by fixed costs, see equation (4).

Finally, there are two labor market clearing conditions. With the above equilibrium con- 
ditions and using the Pareto distribution, they simplify to

$$
M_{i}=p_{i}^{i n} \frac{\rho}{\beta} \frac{L_{i}}{f^{e}}, i \in\{H, F\}
$$

If $p_{i}^{i n}$ were exogenous (or, as in Krugman (1980) equal to unity), the mass of operative firms $M_{i}$ would be proportional to labor supply. Selection decouples this link. Note that with selection, the mass of entrants $M_{i}^{e}=M_{i} / p_{i}^{i n}$ is proportional to endowments.

Summarizing, we have four zero cutoff profit conditions (2), two free entry conditions (5) and two labor market clearing conditions (7) to pin down eight unknown endogenous variables of the model $\left\{\varphi_{H H}^{*}, \varphi_{F F}^{*}, \varphi_{H F}^{*}, \varphi_{F H}^{*} ; M_{H}, M_{F} ; w_{H}, w_{F}\right\}$. Knowledge of these equilibrium objects allows to determine $m_{i j}, p_{i}^{i n}, P_{i}$, and $N_{i}$. In the following, we use Foreign labor as the numeraire. We denote $w_{H} / w_{F} \equiv \omega$ as the relative wage and $\chi \equiv p_{i}^{i n} / p_{j}^{i n}=\left(\varphi_{F F}^{*} / \varphi_{H H}^{*}\right)^{\beta}$ as the relative probability of successful entry and solve the model in relative terms.

Balanced trade is implicit in conditions (2), (5) and (7); it is implied by zero profits of the final good producers. Nonetheless, it is useful to make the balanced trade condition explicit. It can be written as

$$
M_{H} \bar{r}_{H F}=M_{F} \bar{r}_{F H}
$$

where $M_{j} \bar{r}_{j i}$ denotes aggregate sales of intermediate input suppliers located in country $j$ in market $i$. Average sales are given by $\bar{r}_{i j}=\sigma \theta w_{i} m_{i j} f_{i j}$. As argued in case of the free entry condition for profits, average sales are independent of external economies. Using the definition of $m_{i j}$ expression along with equation (6), the trade balance condition (7) can be rewritten so that the relative wage $\omega$ appears as a function of Home's relative endowments and the ratio of the two countries' export productivity cutoffs:

$$
\omega=L^{-1}\left(\frac{\varphi_{F H}^{*}}{\varphi_{H F}^{*}}\right)^{-\beta} \quad \text { with } \quad L \equiv \frac{L_{H}}{L_{H}} .
$$

So, the trade balance condition ensures that, for given relative country size, a shift in $\omega$ will induce opposite movements in two two countries' foreign market access threshold productivities $\varphi_{F H}^{*}$ and $\varphi_{H F}^{*}$. Without loss of generality, we assume Home to be weakly larger than Foreign such that $L \geq 1$. 


\subsection{Total factor productivity}

Total factor productivity (TFP) is a measure of the relative well-being (welfare) of the representative consumer. The reason is that final good consumption enters positively and as the only argument in the utility function of the representative consumer. ${ }^{18}$

In line with Melitz (2003), TFP (and, as argued above, welfare) can be written as

$$
\operatorname{TFP}_{i}=\rho \times N_{i}^{\frac{\mu}{\sigma-1}} \times \tilde{\varphi}_{i}
$$

where $N_{i}$ is the mass of available inputs (scale effect) and $\tilde{\varphi}_{i}$ measures the average productivity of input suppliers. It is defined as the weighted average of domestic and foreign input suppliers' average productivities:

$$
\tilde{\varphi}_{i} \equiv\left\{\frac{1}{N_{i}}\left[M_{i i} \tilde{\varphi}_{i i}^{\sigma-1}+M_{j i}\left(\frac{\tilde{\varphi}_{j i}}{\tau_{j i} w_{j} / w_{i}}\right)^{\sigma-1}\right]\right\}^{\frac{1}{\sigma-1}} .
$$

The weights are the shares domestic and foreign inputs, respectively, in total available inputs. Following Melitz (2003), we define average productivities of input supplies located in country $j$ serving the final good producer in country $i$ as in Melitz (2003) as

$$
\tilde{\varphi}_{j i} \equiv\left[\int_{\varphi_{j i}^{*}} \varphi^{\sigma-1} \frac{d G[\varphi]}{1-G\left[\varphi_{j i}^{*}\right]}\right]^{\frac{1}{\sigma-1}}
$$

It is well known that with Pareto, average productivity is linear in the entry cutoff.

In $\tilde{\varphi}_{i}$, average productivity of foreign input suppliers is corrected for the facts that (i) importing is subject to trade $\operatorname{costs} \tau_{j i}$, and (ii) there is a cost differential $w_{j} / w_{i}{ }^{19}$

Writing equation (9) in relative terms, we eliminate the cost term $\rho$ :

$$
\mathrm{TFP} \equiv \frac{\mathrm{TFP}_{i}}{\mathrm{TFP}_{j}}=N^{\frac{\mu}{\sigma-1}} \times \tilde{\varphi}
$$

\footnotetext{
${ }^{18}$ See Ramondo et al. (2015) on the equivalence of TFP and real income in a model with labor as the only factor of production and without saving.

${ }^{19}$ In the standard Melitz (2003) model with symmetric countries, wages are equalized across countries, and there is no such cost differential.
} 
where $N \equiv N_{i} / N_{j}$ and $\tilde{\varphi} \equiv \tilde{\varphi}_{i} / \tilde{\varphi}_{j}$ denotes the relative mass of available varieties and the relative average productivity.

In models without selection, the mass of available inputs is the same in both countries, resulting in $N=1$. Cross-country differences in TFP are then entirely driven by differences in $\tilde{\varphi}$. As shown by Krugman (1980), the larger country pays the higher nominal wage, reflecting a locational advantage. Due to the presence of (variable) trade costs, the share of expenditure spent on domestically produced inputs is always larger than the share spent on imported inputs. Hence, the nominal wage differential also translates into a real wage differential. This real wage gap cannot be affected by changes in the degree of external economies of scale. Hence, regardless of external of scale, the prediction of the single-sector Krugman (1980) model is at odds with the stylized fact presented in section 2 . We show below that in the Melitz (2003) model, in contrast to the Krugman (1980) model, (i) the relative mass of available varieties is increasing in relative country size, (ii) relative average productivity $\tilde{\varphi}$ is falling in relative country size, and (iii) the latter effect can dominate the former effect, if the degree of external economies of scale is sufficiently low.

The relative mass of available inputs $N$ and relative average productivity $\tilde{\varphi}$ can be linked to the key variables of the subsequent analysis, $\chi$ and $\omega$. Using $M_{j i}=\left(\varphi_{j i}^{*}\right)^{-\beta} M_{j}^{e}$ and the zero cutoff profit conditions, we obtain

$$
N=L \times \chi \times \frac{\frac{f^{x}}{f^{d}}+\eta L^{-1} \omega^{\frac{\beta}{\rho}}}{\frac{f^{x}}{f^{d}}+\eta L \omega^{-\frac{\beta}{\rho}}} .
$$

In models with selection, $N$ depends on endowments and trade costs in a non-trivial way. The first term is a direct effect of relative country size. The second term represents the domestic selection effect. The third term reflects export selection in the foreign country.

Relative average productivity can be rewritten as

$$
\tilde{\varphi}=\left(\frac{L}{N}\right)^{\frac{1}{\sigma-1}} \times \chi^{-\frac{1}{\beta}},
$$

where the first term is a relative weight and the second term is inversely related to the relative entry probability. 


\section{The home market effect with selection and endogenous wages}

We now develop a graphical tool to analyze how relative country scale affects the relative number of firms in different locations, and how this relationship is shaped by trade costs and productivity dispersion.

\subsection{A graphical tool to solve the asymmetric Melitz model}

We characterize the equilibrium of the asymmetric Melitz model with the help of two separate equilibrium conditions in a diagram with the relative wage $\omega$ on the x-axis and the relative probability of successful entry $\chi$ on the y-axis. The ex ante and ex post profitability curve, which we will characterize in detail below, have opposite slopes and allow conducting comparative statics in an insightful and tractable manner. Equilibrium of the two-country asymmetric Melitz economy is given by the intersection of these curves.

Equilibrium values of $\omega$ and $\chi$ do not depend on the degree of external economies of scale $\mu$. The reason is that equilibrium in these variables can be established using the zero cutoff profit conditions in relative form (4), the free entry conditions (5), and the trade balance condition (8), all of which are invariant to changes in $\mu$. External economies of scale play an important role, of course, when it comes to the price index associated to the final good, and to TFP.

Ex post profitability. In order to derive the ex post profitability curve, we start from the trade balance condition (8) and use the zero cutoff profit conditions in relative form (4) to substitute out both export cutoffs. Finally, we employ the definition $\chi \equiv\left(\varphi_{F F}^{*} / \varphi_{H H}^{*}\right)^{\beta}$, and solve for $\chi$ :

$$
\chi[\omega]=L \times \omega^{-\frac{2 \beta-\rho}{\rho}} .
$$

It is easy to check that the ex post profitability curve is downward-sloping and convex. We have $\chi[1]=L$. An increase in $L$ leads to an upward shift in the ex post profitability curve. Moreover, $\chi[\omega]$ converges to zero as $\omega \rightarrow \infty$. Figure 2 illustrates the locus. It takes an ex post perspective in that it summarizes firm behavior after the resolution of uncertainty about pro- 
ductivity. A higher wage constitutes a cost disadvantage, which requires a higher productivity to make non-negative profits. A higher entry cutoff implies a lower probability of successful entry.

Figure 2: Existence and uniqueness of equilibrium

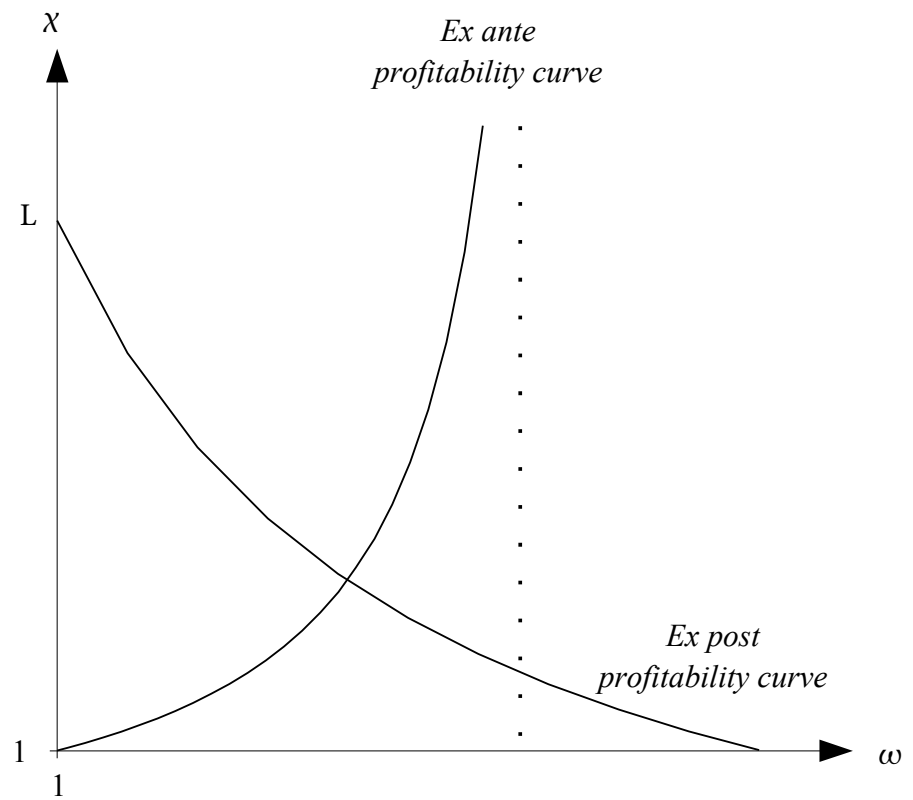

Ex ante profitability. The derivation of the ex ante profitability curve starts from the free entry conditions (5). It makes use of the relative zero cutoff profit conditions (4) in order to eliminate export cutoffs and employs the definition of $\chi \equiv\left(\varphi_{F F}^{*} / \varphi_{H H}^{*}\right)^{\beta}$ to eliminate relative domestic cutoffs. Finally, it solves the resulting equation for $\chi$ :

$$
\chi[\omega]=\frac{1-\eta \omega^{-\frac{\beta}{\rho}}}{1-\eta \omega^{\frac{\beta}{\rho}}}, \eta \omega^{\frac{\beta}{\rho}} \neq 1 .
$$

The ex ante profitability curve emerges from the point $(1,1)$ with a slope $2 \beta /(\rho(1-\eta))$. Moreover, it features an asymptote at $\omega=\eta^{-\rho / \beta}>1$. The curve is convex if $\eta>\rho /(2 \beta+\rho) .{ }^{20}$ Figure 2 illustrates the locus.

\footnotetext{
${ }^{20}$ The condition $\eta>\rho /(2 \beta+\rho)$ guarantees that the second derivative is positive in $\omega=1$. If the ex ante profitability curve is convex in $\omega=1$, it is also convex for $\omega \in\left(1, \eta^{-\frac{\rho}{\beta}}\right)$.
} 
The ex ante profitability curve illustrates a positive relationship the relative entry probability $\chi$ and the relative wage rate $\omega$. It takes an ex ante perspective in that it relates to potential firms' decisions to sink setup costs and learn about their productivities. A higher wage will make it harder to export and therefor lower the export participation rate. Hence, expected profits decline. In order restore free entry, the probability of successful entry must go up.

Lemma 1 (Equilibrium.) Consider a version of the Melitz (2003) model with two countries that potentially differ in size. There exists a unique equilibrium at the intersection between a strictly downward-sloping convex ex post profitability curve

$$
\chi[\omega]=L \omega^{-\frac{2 \beta-\rho}{\rho}}
$$

and a strictly increasing convex (the latter under mild parameter restrictions) ex ante profitability curve:

$$
\chi[\omega]=\frac{1-\eta \omega^{-\frac{\beta}{\rho}}}{1-\eta \omega^{\frac{\beta}{\rho}}}, \eta \omega^{\frac{\beta}{\rho}} \neq 1 .
$$

Proof. In the text.

Note that Lemma 1 resembles the balance-of-payments condition that determines the wage rate in a single-sector model without selection if one equates equations (14) and (15) and considers the limiting case $\beta \rightarrow \sigma-1$; see equation (14) in Krugman (1980).

\subsection{Wage and selection effects}

Figure 2 allows us to analyze the effect of market size on wages. and selection. The following proposition states the result:

Proposition 1 (Wage and selection effects.) Consider a version of a Melitz (2003) model with two countries of different size $(L>1)$. The larger country pays the higher wage $(\omega>1)$ and features a relatively lower selection effect $(\chi>1)$.

Proof. Immediate follows from the observations that the ex ante profitability curve emerges from $(1,1)$ and is increasing with an asymptote at $\omega=\eta^{-\rho / \beta}$, while at $\omega=1$, the downward- 
sloping ex post profitability curve results in $\chi=L>1$ with an asymptote at $\chi=0$ when $\omega$ goes to infinity.

The intuition for the result is simple: At given factor costs, firms find it more profitable to produce in the larger market as this minimizes payments of variable trade and market access costs. As in a single-sector model with homogeneous firms, this advantage is offset by a wage differential. Moreover, presence in the large country is particularly valuable for firms with intermediate productivity levels. Since they do not export, the higher wage in Home puts them at a competitive disadvantage in Home but not in Foreign. Hence, the selection effect is weaker in the larger country.

Note that in the single-sector Krugman (1980) model, there is no selection, and only the wage adjustment channel is active. Hence, the wage effect must be larger in models with homogeneous firms than in models with heterogeneous firms. Note that the welfare consequences in general differ across models. While a higher wage makes domestic varieties more expensive, thereby increasing the price index, the direct effect on labor income dominates such that real income increases. More domestic variety increases TFP as producers benefit from an increased variety of inputs.

\subsection{Home Market Effect}

We now analyze the mapping between relative endowments $L$ and the relative mass of active firms. Let $M \equiv M_{H} / M_{F}$ denote this relative mass of active firms. Following the literature, we define a Home Market Effect (HME) as follows: ${ }^{21}$

Definition 1 A Home Market Effect (HME) exists, if the relative mass of active firms located in a country $M$ is larger than its relative endowments $L$ :

$$
M=\gamma \times L, \text { where } \gamma>1 .
$$

\footnotetext{
${ }^{21}$ The definitions used by Helpman and Krugman (1985), Hanson and Xiang (2004) or Behrens, Lamorgese, Ottaviano, and Tabuchi (2009) coincide with it.
} 
Hence, a HME exists, if a countries hosts a share of active firms that is larger than its endowment share would predict. ${ }^{22}$ In the case with a linear outside sector (with or without firm-level heterogeneity), $\gamma$ is equal to a constant $\bar{\gamma}>1$, such that a HME obtains. ${ }^{23}$

Employing equation (6), we find that in the single-sector Melitz (2003) model, the relative mass of active firms $M$ is proportional to relative endowments times the relative probability of successful entry:

$$
M=\chi \times L
$$

Hence, the model exhibits a HME, if and only if the probability of successful entry $\chi$ is greater in the larger home economy than in Foreign, i.e., $\chi>1$. Figure 2 establishes that this is indeed the case.

Note the crucial role of firm-level heterogeneity: if all firms were identical, and thus, in a meaningful equilibrium, ${ }^{24}$ all of them would find it worthwhile to produce, in both countries we would have $p_{i}^{i n}=1$, and hence $\gamma=1$. The relationship between the relative mass of active firms and relative endowments would be one-to-one: there would not be a HME. ${ }^{25}$

These considerations allow stating the second key proposition of this paper:

Proposition 2 (Home Market Effect). Consider a two-country Melitz (2003) model where countries differ in size $(L>1)$. The larger country hosts an over-proportional mass of active firms:

$$
M>L
$$

Proof. In the text. It builds on the relationship $M=\chi \times L$ and the observation that the larger country features a relatively higher probability of successful entry due to a weaker selection effect, i.e., $\chi>1$.

\footnotetext{
${ }^{22}$ It is easy to check that $M>L \Leftrightarrow M_{H} /\left(M_{H}+M_{F}\right)>L_{H} /\left(L_{H}+L_{F}\right)$.

${ }^{23}$ See Appendix D.3 for the model with a linear outside sector.

${ }^{24}$ The parameter constellation could be such that no firm wants to operate $\left(p_{i}^{i n}=0\right)$. We exclude such shutdown equilibria.

${ }^{25}$ Note that in the Melitz (2003), the relative mass of potential entrants $M_{i}^{e} / M_{j}^{e}$ is directly proportional to relative endowments $L$.
} 
Figure 3: Home Market Effects with and without an outside sector
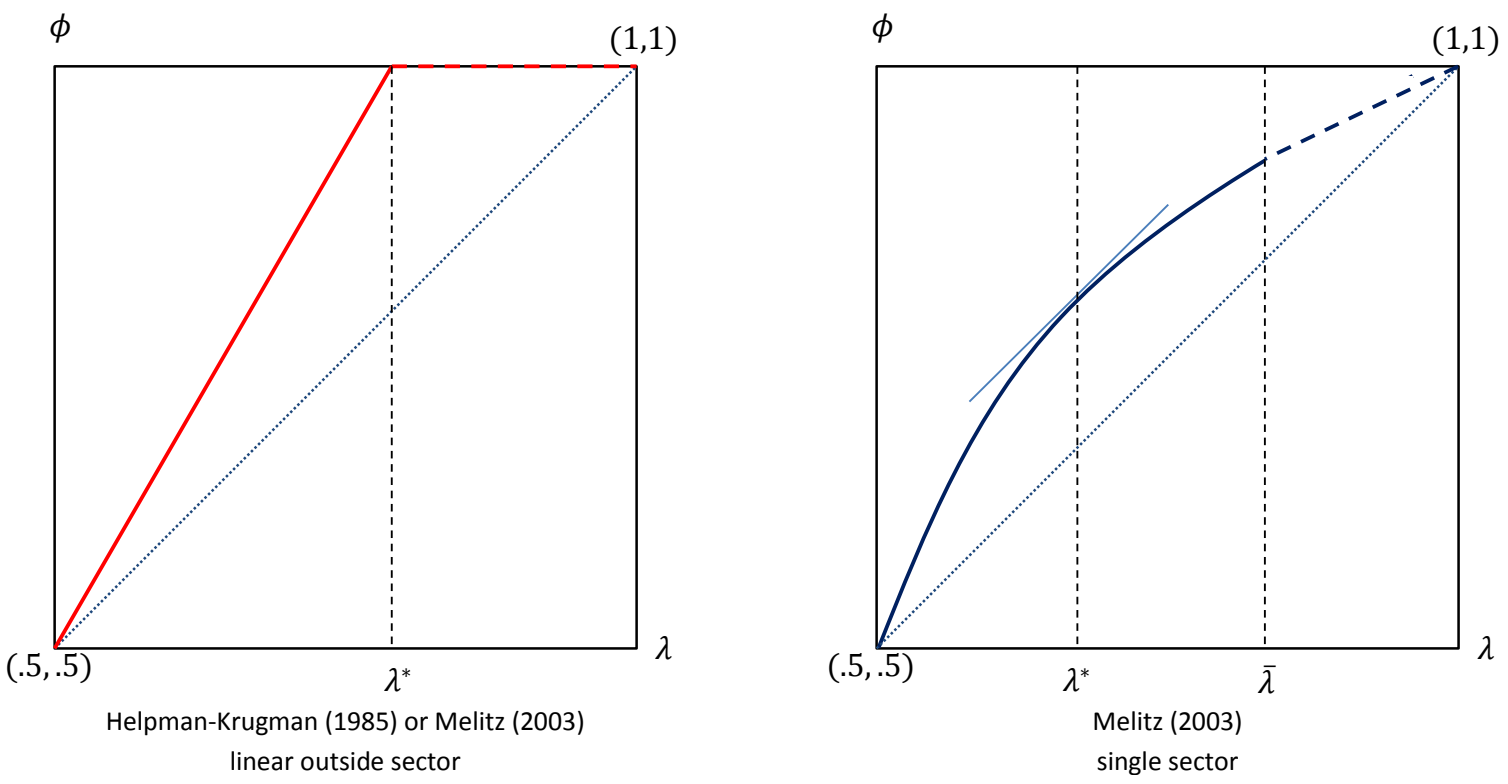

Figure 3 illustrates the HME in models with and without a linear outside sector. It plots the large country's share of active firms $\phi \equiv M_{H} /\left(M_{H}+M_{F}\right)$ as a function of the large country's endowment share $\lambda \equiv L_{H} /\left(L_{H}+L_{F}\right)$.

The diagram on the left hand is the standard illustration of the HME in the HelpmanKrugman (1985) world. It also holds in the Melitz (2003) model with a linear outside sector. ${ }^{26}$ The firm share $\phi$ is always larger than the endowment share $\lambda$. In the interval $\lambda<\lambda^{*}$, the firm share increases more than proportionately in the endowment share. When $\lambda>\lambda^{*}$, the large economy is fully agglomerated $(\phi=1)$, so that a further increase in the large country's endowment share cannot further increase the firm share $\phi$ anymore.

The diagram on the right hand side illustrates the HMEs in the single-sector Melitz case. Clearly, the large country's firm share is larger than its endowment share over the entire interval $\lambda \in(1 / 2,1)$. In the interval $\lambda \in\left(1 / 2, \lambda^{*}\right)$, the firm share increases more than proportionally in the endowment share. ${ }^{27}$ If the large country becomes very large, intermediate input producers in the small country with intermediate productivity levels can afford serving the large country (export market), but not the small domestic market. The small country 's sorting

\footnotetext{
${ }^{26}$ See Appendix D.3 for the model with a linear outside sector.

${ }^{27}$ Technically, $\lambda^{*}$ is found by setting the derivative of $\phi[\lambda]$ with respect to $\lambda$ equal to unity.
} 
condition is violated when $\lambda>\bar{\lambda}$, where the functional relationship between the firm share and the endowment share has a kink. ${ }^{28}$

It can be shown that the firm share $\phi$ is concave in the endowment share $\lambda$ in the interval $(1 / 2, \bar{\lambda})$, but convex in the interval $(\bar{\lambda}, 1)$. Moreover, one can show that the existence of a linear outside sector in the Melitz (2003) model magnifies the HME relative to the case where the outside sector is absent. ${ }^{29}$ In particular, in the case with the outside sector, the slope of the locus $\phi[\lambda]$ is equal to $1+2 \eta$ over the interval $\lambda \in\left(1 / 2, \lambda^{*}\right)$. In the single-sector case, it is equal to $1+\eta /(2-\rho(1-\eta) / \beta)$ in the neighborhood of a symmetric equilibrium with $\lambda=1 / 2<1+2 \eta$, where the inequality follows from the observation that $\beta / \rho>1>1-\eta$.

\subsection{Home Market Magnification Effects}

We now investigate how the strength of the HME is affected by the freeness of trade and by the extent of productivity dispersion. In the traditional Helpman-Krugman case where wages are fixed due to the presence of an outside sector, lower variable trade costs make the HME more pronounced. They also make it more likely that the model degenerates to full agglomeration (where Home has all firms). This home market magnification effect also exists in our case; only it appears in a somewhat subtler form due to the absence of a linear outside sector. We also show that increased dispersion of productivity magnifies the HME.

Trade liberalization. Recall that freeness of trade is defined as $\eta \equiv \tau^{-\beta}\left(f^{x} / f^{d}\right)^{1-\beta /(\sigma-1)}$. It falls when variable trade costs $\tau$ shrink and/or when foreign entry costs relative to domestic ones $f^{x} / f^{d}$ fall. The precise origin of a change in $\eta$ does not matter for the result. Conveniently, $\eta$ only appears in the ex ante profitability curve but not in the ex post profitability crowding curve. Using Figure 2, the comparative statics with respect to $\eta$ are therefore very easy. Figure 4 provides an illustration.

The intuition is the following. For given wages and domestic entry cutoffs, higher freeness

\footnotetext{
${ }^{28}$ The model can still be solved when the sorting condition is violated; see Appendix D.2. One has to take into account that a firm in the small country becomes active, if its productivity level suffices to serve the large country, which has implications for the entry probability and the mass of active firms.

${ }^{29}$ For a discussion of the Melitz (2003) model with outside sector, see Appendix D.3.
} 
Figure 4: Trade liberalization

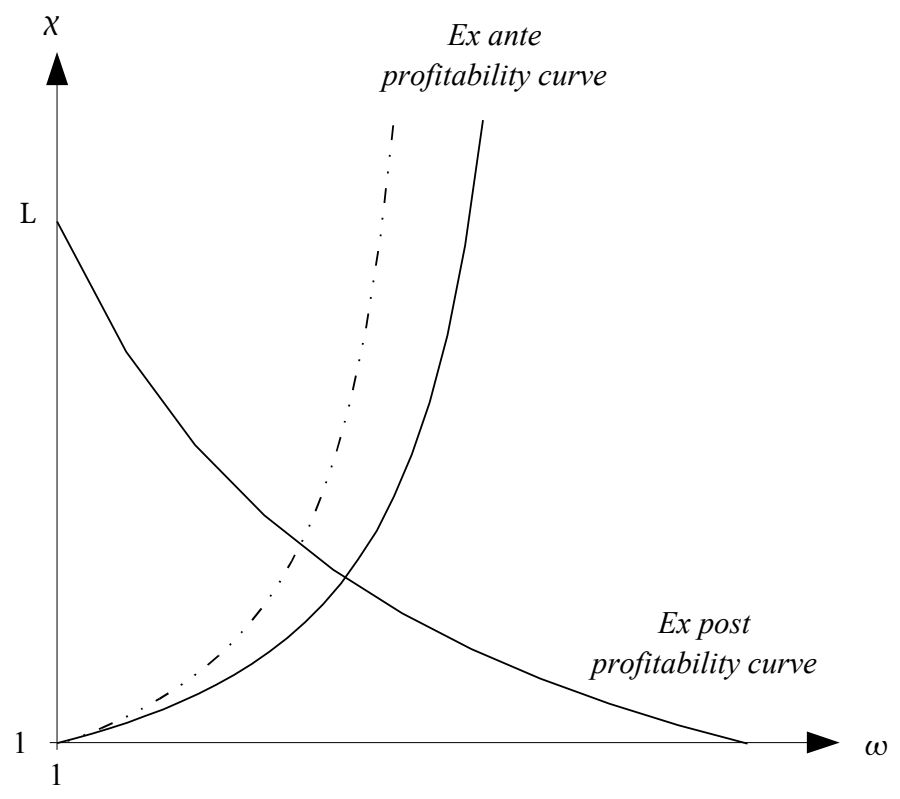

of trade increases the export participation rate and therefore expected profits in both countries. Free entry implies that the probability of successful entry has to go down in both countries. A symmetric drop in trade costs favors the smaller country, which is more open and now has easier access to the large market, which implies that the relative entry probability from the large country's perspective rises in response to symmetric trade liberalization. The ex post profitability locus is not affected since freeness of trade rises symmetrically. Hence, the equilibrium relative entry probability goes up, which translates into a larger HME since $\gamma$ rises in $\chi$ for given $L$. Moreover, the equilibrium relative wage declines, so that higher freeness of trade leads to convergence of nominal wages.

The equilibrium relative entry probability $\chi$ is also concave in the freeness of trade $\eta$. The reason is that (i) the ex post profitability curve is convex in the relative wage, (ii) it is not shifted itself by a freeness of trade shock, and (iii) the relative wage is strictly decreasing in the freeness of trade.

Productivity dispersion. Next, we consider the comparative statics with respect to $\beta$, the shape parameter of the Pareto distribution, which is inversely related to the variance of the sampling distribution. $\beta$ appears in both the ex ante and the ex post profitability curves. 
An increase in productivity dispersion (lower $\beta$ ) rotates both curves upwards. Hence, higher productivity dispersion unambiguously leads to a higher relative entry probabilities $\chi$, while the effect on the wage rate depends on model parameters in a complicated fashion. A higher $\chi$ magnifies the home market effect; see proposition 2 .

For a given relative wage, higher productivity dispersion reduces the effective cost differential. Hence, the relative domestic entry cutoff from the larger country's perspective falls. This results in an increase in the relative entry probability and is reflected by an upward rotation of the ex post profitability curve.

For given trade costs, lower productivity dispersion lead to lower effective trade costs. Lower trade costs favor the smaller, more open country. Free entry implies that the relative entry probability from the larger country's perspective must go up, resulting in the upward rotation of the ex ante profitability curve. ${ }^{30}$ Both rotations work in favor of a higher relative entry probability from the larger country's perspective. Hence, higher productivity dispersion relatively weakens the selection effect in the larger country. ${ }^{31}$ So, for the emergence of a HME in the single-sector Melitz model, productivity dispersion is important. ${ }^{32}$

The following proposition summarizes this third key result of our analysis.

Proposition 3 (Home Market Magnification Effects.) Consider a two-country Melitz (2003) model where countries differ in size ( $L>1$ ). The home market effect is magnified by trade liberalization and an increase in productivity dispersion (lower $\beta$ ):

$$
\frac{\partial \chi}{\partial \eta}>0 \text { and } \frac{\partial \chi}{\partial \beta}<0
$$

Proof. The effect of trade liberalization on $\chi$ follows from noting that (i) the ex post profitability curve is invariant to changes in $\eta$ and (ii) the ex ante profitability curve rotates upwards in

\footnotetext{
${ }^{30}$ Equation (14) shows that productivity dispersion also has an effect on the effective cost differential, but this effect is dominated by the freeness-of-trade effect.

${ }^{31}$ Our graphical analysis shows that $\left(\varphi_{F F}^{*} / \varphi_{H H}^{*}\right)^{\beta}$ goes up when $\beta$ becomes smaller. Hence, $\varphi_{F F}^{*} / \varphi_{H H}^{*}$ must go up, which implies that selection becomes relatively weaker in the larger country.

${ }^{32}$ This finding has important implications for empirical studies on the HME, such as Hanson and Xiang (2004). The model suggests that one important industry characteristic that shapes the size of the HME is the degree of productivity dispersion as captured by the shape parameter of the Pareto distribution.
} 
Figure 5: Higher productivity dispersion

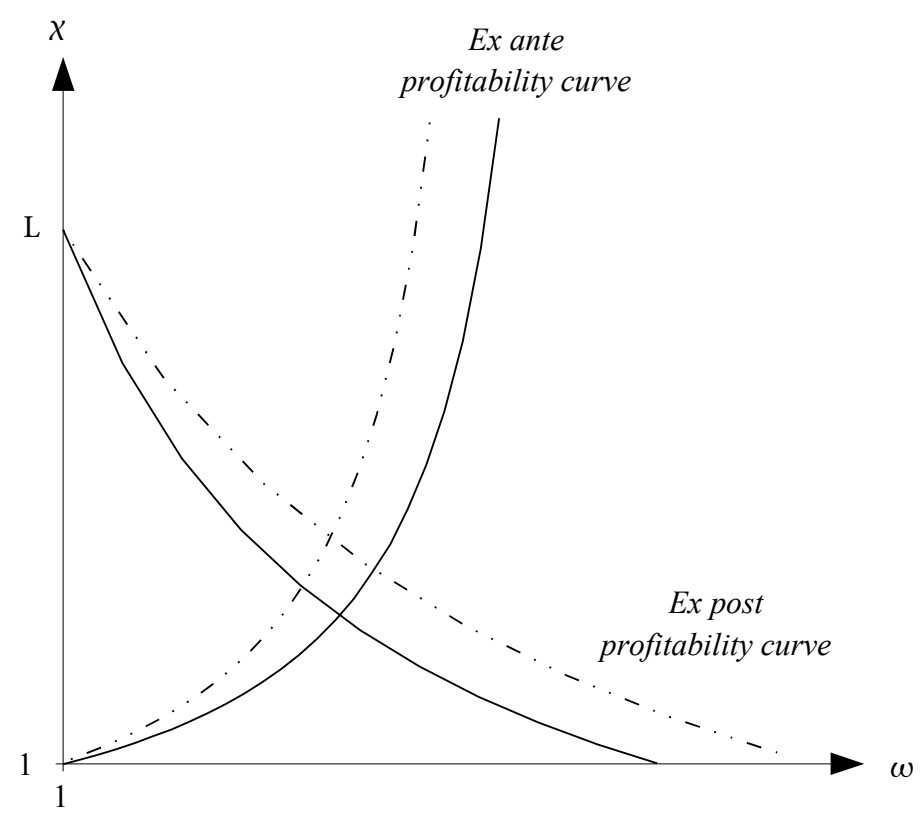

response to an increase in $\eta$ around the point $(1,1)$ as

$$
\frac{\partial \chi}{\partial \eta}=\frac{\omega^{\frac{\beta}{\rho}}-\omega^{-\frac{\beta}{\rho}}}{\left(1-\eta \omega^{\frac{\beta}{\rho}}\right)^{2}}>0 .
$$

The inequality follows from $\omega>1$. Recall from Proposition 2 that an increase in $\chi$ directly translates into an increase in the HME. The proof for the effect of productivity dispersion on the equilibrium value of $\chi$ is more complicated and relegated to Appendix C.1.

\section{Scale and total factor productivity}

The HME links the global distribution of firms to relative country size. EoS do not matter for this; however, to the extent that the HME also implies a better availability of inputs, it will also matter importantly for relative TFP. In models without selection, the link would be trivial: all inputs are used in all countries, although, of, course, everything else equal, demand for imported inputs will be lower than demand for domestic inputs due to variable trade costs. Thus, external EoS would be irrelevant for the link between relative scale and relative TFP. In this section, we study what implications the HME has on relative TFP in a model with 
selection and flexible degrees of external economies of scale.

\subsection{Relative mass of available varieties}

Consider the definition of $N$ in equation (12). We have $N / L>1$, if the selection effect dominates the indirect effect that comes through export selection. It can be shown that this indeed the case. We therefore state the following proposition:

Proposition 4 (Available inputs.) Consider a two-country Melitz (2003) model where countries differ in size and $f^{x} \geq f^{d}$. The larger country has access to an over-proportional mass of inputs

$$
N>L
$$

Proof. In order to prove the result, we start from equation (12) and multiply out $\chi$ and the term $\frac{\frac{f^{x}}{f^{d}+\eta L^{-1} \omega^{\frac{\beta}{\rho}}}}{\frac{f^{x}}{f^{d}}+\eta L \omega^{-\frac{\beta}{\rho}}}$. The product of these terms is larger than 1 , if the equilibrium wage is sufficiently small. Using the ex post profitability curve and the result $\chi>1$, we show that this restriction is always met; see Appendix C.2 for a detailed proof.

The proposition implies that in the presence of selection, the larger country has access to an over-proportional mass of inputs. Two observations stand out: First, the available inputs advantage of the large country translates into relatively higher TFP, if there are external economies of scale. Although the Home Market Effect in domestic inputs is not directly linked to TFP, it should be noted that the Home Market Effect in domestic inputs is the driving force behind the advantage in available inputs as it dominates the countervailing effect through export selection. Second, we have identified a channel through which relative market size positively affects relative TFP. This seems to be at odds with the stylized facts presented in section 2. Note, however, that how this size advantage translates into a relative TFP gap depends on the degree of external economies of scale. 


\subsection{Average productivity}

We now study the effect of market size on average productivity. One way to express average productivity, which allows to nest models with and without selection, is:

$$
\tilde{\varphi}=N^{-\frac{1}{\sigma-1}} \chi^{\frac{1}{\sigma-1}-\frac{1}{\beta}} \omega^{\frac{2 \beta-\rho}{\rho(\sigma-1)}} .
$$

Average productivity can be understood as the product of a (i) scale (variety) effect $N^{-\frac{1}{\sigma-1}}$ that relates to the presence of firm-level economies of scale, a (ii) selection effect that relates to the fact that drives the mass of domestic and imported varieties available (relative to potential entrants) and the average productivities of domestic and imported varieties (this is a HME in terms of relative entry probability), and (iii) the canonical HME in wages.

In the limiting case $\beta \rightarrow \sigma-1$, which effectively shuts down the selection mechanism and therefore yields the Krugman (1980) setup, we have

$$
\tilde{\varphi}=\omega^{\frac{2 \sigma-1}{\sigma-1}} \geq 1
$$

where the inequality follows from proposition 1 and $\sigma>1$. Hence, in the absence of selection, market size exhibits a positive effect on average productivity. The reason is that with an increase in domestic market size, relatively more inputs come without (variable) trade costs.

With selection, this mechanism is active as well, but counteracted by the fact that higher market size weakens domestic selection so that less productive firms remain active. We show in this section that the anti-selection effect dominates, establishing a negative link between market size and average productivity.

Recall that relative average productivity can be written as

$$
\tilde{\varphi}=\chi^{-\frac{1}{\beta}} \times\left(\frac{N}{L}\right)^{-\frac{1}{\sigma-1}} \leq 1 .
$$

The direct domestic selection effect is accompanied by a weighting factor. We have established in section 4.2 that the larger country exhibits are relatively weaker domestic selection effect, resulting in a negative effect of market size on relative average productivity. More- 
over, we have seen in the previous section that the larger country has access to an overproportional mass of inputs. This link further reduces average productivity.

We can state the following proposition:

Proposition 5 (Average productivity.) Consider a two-country Melitz (2003) where countries differ in size. The larger country has the lower average productivity:

$$
\tilde{\varphi}<1 \text {. }
$$

Proof. Immediately follows from the observations that $\chi>1$ (Proposition 1 ) and that $N / L>$ 1 (Proposition 4).

\subsection{Total factor productivity}

In this subsection, we analyze the role of market size for relative total factor productivities (TFP) of the final good producers.

Models without selection. In models without selection, relative TFP (the cross-country gap in living standards) is simply determined by the relative wage, see equation (18). As the larger country pays the higher wage (proposition 1), it enjoys the larger TFP. An increase in the variance of the endowment distribution leads to an increase in the variance of the TFP distribution. ${ }^{33}$ Trade liberalization reduces the wage differential (Figure 4) and therefor leads to convergence of relative TFPs. Trade liberalization can never put the large country into a position where it exhibits lower TFP than the smaller country.

TFP in the Melitz model. Using equation (13) to substitute out $\tilde{\varphi}$ from equation (11), we can rewrite relative TFP as a function of the exogenous country size differential and endogenous variables that have played a key role in our analysis so far and whose properties are well

\footnotetext{
${ }^{33}$ Note that there is a unique mapping between the endowment share of the large economy $\lambda$ and relative country size $L$ given by $\lambda=\left(1+L^{-1}\right)^{-1}$.
} 
understood, namely $\chi$ and $N$ :

$$
\mathrm{TFP}=N^{\frac{\mu}{\sigma-1}} \underbrace{\left(\frac{N}{L}\right)^{-\frac{1}{\sigma-1}} \chi^{-\frac{1}{\beta}}}_{=\tilde{\varphi}<1} .
$$

Standard CES case. In order to understand the different components of relative TFP, consider first the standard CES with $\mu=1$. Then, the scale effect exactly offsets the weighting effect of the mass of available inputs on average productivity, and the TFP expression simplifies to $\mathrm{TFP}=L^{\frac{1}{\sigma-1}} \chi^{-\frac{1}{\beta}}$. We know from Figure 2, that $\chi<L$. Together with the condition $\beta>\sigma-1$, this ensures that TFP $>1$. Hence, the positive scale effect more than offsets the negative effect of scale on average productivity effect. The effect induced by having more entrants dominates the weaker selection effect, such that the larger Home has the higher TFP. It is easy to see that TFP increases with $L$ so that a more unequal distribution of the world labor endowment leads to more disparity in terms of welfare.

For given relative country size, an increase in the freeness of trade raises $\chi$ and therefore lowers TFP. In other words, in the standard CES case, trade liberalization leads to convergence of welfare across countries towards TFP $=1$. The intuition is that higher freeness of trade favors the more open country, which is the small country. In the model, convergence of real income is equivalent to factor price convergence.

Importantly, trade liberalization does not affect relative real per capita income in models with exogenous wages. ${ }^{34}$ The intuition for this result lies in the simple fact that fixing the wage rate also fixes mill prices in a CES environment. So, trade liberalization could affect TFP differently across countries, if cutoff productivity levels were differently affected by a change in $\eta$. This is, however, not possible when wages are insensitive to $L$ and $\eta$.

We have argued above that the presence of a linear outside sector exaggerates the importance of the HME. Interestingly, one can show that this does not imply that the cross-country welfare differential must be bigger, too. Quite the opposite is true. The welfare differential is larger in the absence of the outside sector.

\footnotetext{
${ }^{34}$ See Appendix D.3.
} 
The following proposition summarizes our results for the standard CES case:

Proposition 6 (Relative TFP in the standard CES case.) Consider a two-country Melitz (2003) model where countries differ in size and where the degree of external economies of scale in the final good production function is the one implied by the standard CES case $(\mu=1)$. Then,

(a) the larger economy exhibits the higher TFP, and

(b) trade liberalization leads to TFP convergence across countries.

Proof. In the text.

The role of external economies of scale. Next, turn to a situation where economies of scale are smaller than implied by the standard CES case. In the polar case where external economies of scale are entirely absent, i.e., $\mu=0$, TFP is determined by average productivity, i.e., $\mathrm{TFP}=$ $\tilde{\varphi}=(L / N)^{1 /(\sigma-1)} \chi^{-1 / \beta}$. As we have argued above, the larger country has the lower average productivity as it features (i) a weaker selection effect such that average productivity of domestic input suppliers is lower and (ii) domestic inputs have more weight in average productivity as the larger country has access to relatively more domestic inputs. These observations imply that the relatively larger country has the relatively lower TFP, i.e., $\mathrm{TFP}<1$. In the polar case $\mu=0$, relative TFP is decreasing in relative market size.

Note that this prediction relies on selection. With selection, the larger country has a weaker selection effect, which reduces relative TFP. Moreover, the direct size advantage is more than offset by the fact that the larger country has access to more inputs, which puts an extra weight on the low average productivity of domestic input suppliers and therefore further reduces average productivity. In the absence of selection, TFP is always increasing in relative market size, regardless of the degree of economies of scale.

Next, consider situations where $\mu \in(0,1)$. By continuity of relative TFP in $\mu$, there must exist a $\bar{\mu}$ for which relative TFP is invariant to relative market size. It is implicitly defined by

$$
T F P=N^{\frac{\bar{\mu}}{\sigma-1}} \tilde{\varphi} \stackrel{!}{=} 1
$$

The following proposition summarizes the role of external economies of scale for relative TFP: 
Proposition 7 (Role of external economies of scale.) Consider a two-country Melitz (2003) model where countries differ in size. Let $\bar{\mu}$ be the threshold degree of external economies of scale at which TFP is independent of scale

$$
0<\bar{\mu}=\frac{\ln N-\ln L+\frac{\sigma-1}{\beta} \ln \chi}{\ln N}<1
$$

Then,

(a) The larger country exhibits the larger TFP, if $\mu>\bar{\mu}$,

(b) Relative TFP is invariant to relative country size, if $\mu=\bar{\mu}$, and

(c) The larger country exhibits the smaller TFP, if $\mu<\bar{\mu}$.

Proof. We obtain the above expression by taking logs on both sides of equation (20) and solving for $\bar{\mu}$. As $N / L>1$ and $\chi>1, \bar{\mu}$ must be positive. As $\chi<L$ and $\beta>\sigma-1$, we have $\frac{\sigma-1}{\beta} \ln \chi-\ln L<0$, which proves $\bar{\mu}<1$.

Clearly, part (a) of the proposition includes the standard CES case. The empirical relevant case where there is no or a negative link between welfare and country size is covered, respectively, by parts (b) and (c) of the proposition. Hence, a Melitz (2003) model that allows for sufficiently small external economies of scale can be used to derive a prediction on the welfare-size nexus consistent with the data.

An increase in the variance of the endowment distribution leads to an increase in the variance of the TFP distribution in the standard CES case, where the larger country has the larger TFP. Proposition 7 implies that this result holds as long as $\mu>\bar{\mu}$. In cases where $\mu<$ $\bar{\mu}$, the smaller country exhibits the larger TFP, and this differential increases in relative size. Hence, also with $\mu<\bar{\mu}$, an increase in the variance of the endowment distribution leads to an increase in the variance of the TFP distribution, although, as argued above, the smaller country gets richer.

Clearly, $\bar{\mu}$ will be a function of trade costs. It might happen that a country that finds itself in a position where it enjoys higher TFP before trade liberalization exhibits lower TFP after trade liberalization. This happens if a given $\mu$ is larger than the $\bar{\mu}$ that obtains before and lower than the $\bar{\mu}$ that obtains after trade liberalization. In the neighborhood of a symmetric 
equilibrium, $\bar{\mu}$ is given by: ${ }^{35}$

$$
\bar{\mu}=2 \eta \frac{(\beta+\sigma-1)\left(\frac{f^{x}}{f^{d}}+\eta\right)+(\beta+\rho)(1-\eta)-2 \beta}{[2 \beta(1+\eta)-\rho(1-\eta)]\left(\frac{f^{x}}{f^{d}}+\eta\right)+2 \eta(\beta+\rho)(1-\eta)-4 \beta \eta} .
$$

\subsection{Numerical exercise}

In order to gauge the importance of the different TFP components and of the external economies of scale, we perform two simple numerical exercises with two countries. The parametrization follows Balistreri, Hilberry, and Rutherford (2011). In line with the findings of Bernard, Eaton, Kortum, and Jensen (2003), the elasticity of substitution is set to $\sigma=3.8$. Moreover, the shape parameter of the Pareto distribution is set to $\beta=4.582$ to replicate an elasticity of variable trade costs in distance of 0.27. We follow Segerstrom and Sugita (2015) in setting $\tau=1.3$. Then, $f^{x} / f^{d}=1.37$ match the empirically observed US export participation rate of $18 \%{ }^{36}$ The parametrization implies a freeness of trade $\eta \equiv \tau^{-\beta}\left(\frac{f^{x}}{f^{d}}\right)^{1-\frac{\beta}{\sigma-1}}$ of about 0.25 .

Figure 6 plots TFP and its components for different large country's population shares and different values of $\mu$; see equation (9). ${ }^{37}$ The left panel illustrates the benchmark parametrization. In the right panel, we set $\tau=1$. All other parameters remain at their benchmark values. The implied freeness of trade in the liberalization scenario is $\eta \approx 0.82$. Comparing the two panels allows for analyzing the effects of trade liberalization.

The upward-sloping solid lines represents the scale effect $N^{\frac{1}{\sigma-1}}$ for the standard CES case $(\mu=1)$. If one is willing to assume that $\mu \geq 1$, these curves are upper bounds for relative TFP that ignores the negative average productivity effect. For lower values of $\mu$, the scale effect curves rotate inwards. The downward-sloping solid lines represent the average productivity effect. They also reflect relative TFP for the polar case $\mu=0$. Hence, if one assumes that there are no diseconomies of scale, the curves represent a lower bound of relative TFP. The

\footnotetext{
${ }^{35}$ In order to derive the expression, we compute the elasticity of TFP in relative country size in the neighborhood of symmetric equilibrium, set the resulting expression equal to zero, and solve for $\bar{\mu}$; see Appendix D.1 for details.

${ }^{36}$ With two symmetric countries, the export participation rate is $\left(\varphi_{d}^{*} / \varphi_{x}^{*}\right)^{\beta}=\tau^{-\beta}\left(\frac{f^{x}}{f^{d}}\right)^{-\frac{\beta}{\sigma-1}}$.

${ }^{37}$ Having the population share rather than relative country size on the $\mathrm{x}$-axis guarantees that the effects are not driven by an increase in world population.
} 
dashed lines represent relative TFP for the standard CES case $\mu=1$. They are upward-sloping as the positive scale effect (upward-sloping solid line) dominates the negative average productivity effect (downward-sloping solid line). Then, the TFP differential is about $13 \%$ for the benchmark calibration. The dotted lines represent relative TFP for an intermediate degree of external economies of scale $(\mu=0.5) .^{38}$

First, in order to illustrate the role of external economies of scale, consider a situation where one country is $50 \%$ larger than the other, i.e., the large country's population share is $60 \%$. Then, the TFP differential is $13 \%$ in the standard CES case $(\mu=1)$ and $5 \%$ for $\mu=0.5$. As argued above, it becomes negative if $\mu$ is small enough.

Second, we turn to the effects of trade liberalization. The theory predicts that trade liberalization relatively weakens the selection effect in the large country, resulting in an increase in $\chi$. The direct effect of this increase in $\chi$ is an increase in the number of available varieties (see equation (12)) and a decline in average productivity. On net, trade liberalization results in convergence of relative TFP, if $\mu=1$, see proposition 6. In our simulation exercise, the TFP differential turns negative for $\mu=0.5$, see panel (b) of Figure 6. Hence, it might happen that a country with a higher TFP level in an initial situation exhibits relatively lower TFP after trade liberalization. This reversal occurs if $\mu>\bar{\mu}_{0}$ in the initial situation, but $\mu<\bar{\mu}_{1}$ after liberalization. Hence, in models with a flexible degree of external economies of scale, trade liberalization can lead to divergence of TFP levels.

Figure 7 shows the degrees of external economies of scale $\bar{\mu}$ required to obtain make TFP independent of country size in the neighborhood of a symmetric equilibrium for different values of key parameters such as the freeness of trade $\eta$, an inverse measure of the degree of productivity dispersion $\beta$, and the elasticity of substitution, $\sigma .{ }^{39}$ In the left-most panel, the solid line represents $\bar{\mu}$ for a situation in which changes in the freeness of trade come from changes in variable trade costs for $f^{x}=f^{d}$. The dashed line represents $\bar{\mu}$ for a situation in which changes in the freeness of trade stem from changes in relative export costs for $\tau=1$.

If either variable or fixed trade costs are prohibitively high such that the countries are

\footnotetext{
${ }^{38}$ The intermediate case assumes a value of $\mu$ close to the estimate reported in Ardelean (2007).

${ }^{39}$ Figure 9 in Appendix B shows that $\bar{\mu}$ does not vary much with changes in population shares.
} 
Figure 6: Relative TFP and its components as a function of relative scale
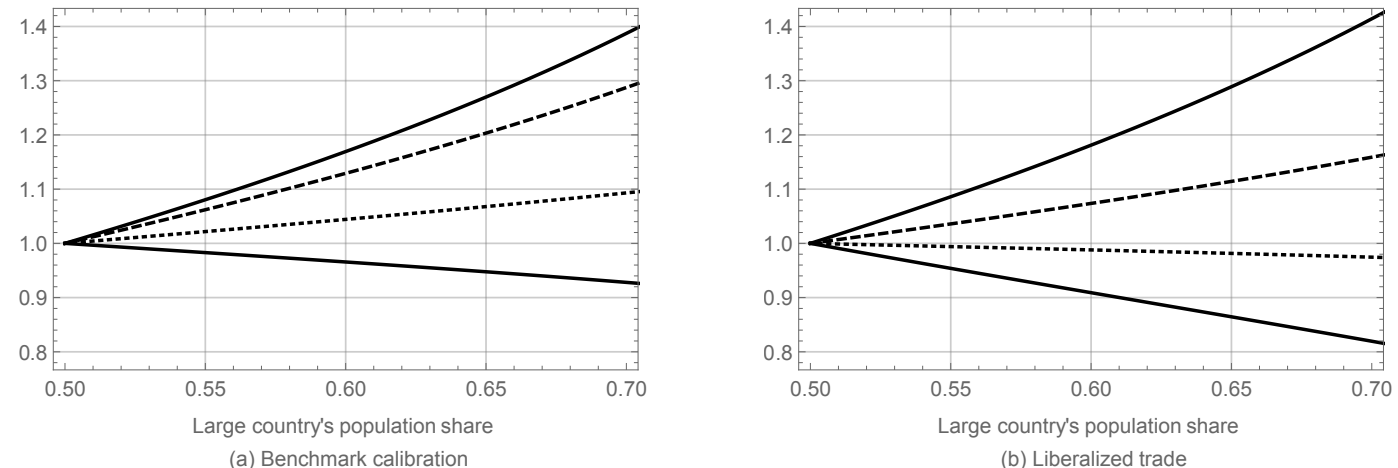

(b) Liberalized trade

Notes: The upward-sloping solid represent the scale effect $N^{1 /(\sigma-1)}$ for the standard CES case $\mu=1$. The downward-sloping solid lines represent the average productivity effect, which is equivalent to relative TFP, if $\mu=0$. The dashed lines represent relative TFP for the standard CES case $\mu=1$ ), and the dotted lines relative TFP for the intermediate case $\mu=0.5$. In the scenario with liberalized trade, variable trade costs are set to $\tau=1$.

Figure 7: Threshold external economies of scale $\bar{\mu}$ as a function of $\eta, \beta$ and $\sigma$
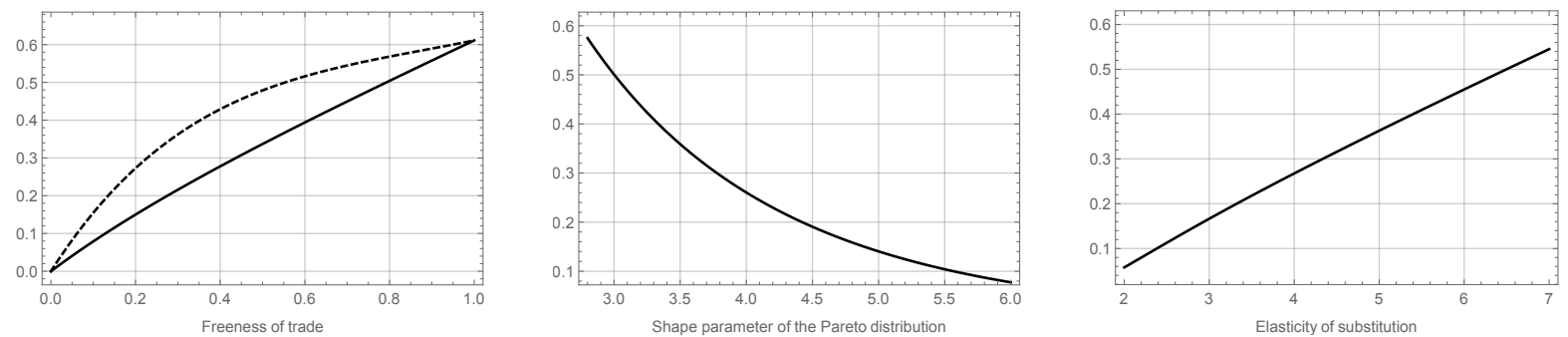

Notes: In all panels, we plot the degree of external economies of scale $\bar{\mu}$ that makes relative TFP independent of relative country size on the $y$-axis. In the left-most panel, the $x$-axis varies the degree of freeness of trade $\eta \equiv \tau^{-\beta}\left(f^{x} / f^{d}\right)^{1-\frac{\beta}{\sigma-1}}$. Variations in $\eta$ stem from variations in either variable trade costs $\tau$ for $f^{x}=f^{d}$ (solid line) or relative export fixed costs $f^{x} / f^{d}$ for $\tau=1$. In the middle panel, the $x$-axis varies the shape parameter of the productivity distribution $\beta$ (inversely related to the degree of productivity dispersion), and in the right-most panel, the $x$-axis varies the elasticity of substitution. In all panels, all other parameters than those varied on the $x$-axis are kept constant to the benchmark parametrization.

closed $(\eta=0)$, the larger country exhibits a larger TFP unless there are diseconomies of scale $(\bar{\mu}=0)$. In the limiting case $\eta \rightarrow 1$, a population share shock has no effect on relative TFP, if $\mu \approx 0.6$. Hence, the absence of a positive effect of relative country size on relative TFP is consistent with data as long as the degree of external economies of scale is sufficiently small. Notice that a given change in the freeness of trade $\eta$ has the same effect on the relative entry probability $\chi$, regardless of whether variable or fixed trade costs are reduced; see proposition 3. Fixed cost liberalization, however, has an additional effect on the mass of available inputs, see equation (12), which feeds into the scale and the average productivity effect.

The middle panel of Figure 7 shows that the critical value of $\mu$ depends negatively on $\beta$. 
Therefore, a higher degree of productivity dispersion amongst firms makes is consistent with stronger external economies of scale. This is intuitive, as a more dispersed firm population weakens selection, so that relative average productivity falls faster in relative scale. Finally, the right-most panel of Figure 7 shows that higher elasticities of substitution go together with higher critical values of $\mu$. Summarizing, with an active selection channel, the model is able to replicate a zero correlation between TFP and scale with degrees of external economies of scale that are positive - confirming intuition - but not considerably smaller than the value of unity conventionally assumed in Dixit-Stiglitz aggregate production functions.

\section{Conclusion}

Empirical evidence shows that aggregate outcomes such as TFP or real per capita income do not increase in country size as semi-endogenous growth models or trade models with trade costs, product differentiation, and free entry would imply. While the growth literature has developed alternative frameworks that do not exhibit the counterfactual implication, trade theorists are only starting to work on it. In this paper, we show that trade models with firm-level productivity dispersion and fixed foreign market entry costs can be in line with this evidence as long as the degree of external economies of scale is not too large. With this variation of the standard Melitz (2003) model one does not have to give up the widely popular notion that a larger product space is beneficial for TFP and per capita income, nor does one have to discard the home market effect (HME) - the over-proportionate link between relative size and the relative share of firms which enjoys some empirical support.

We make this argument with the help of a two-country single-sector asymmetric Melitz (2003) model. We provide a tractable way to characterize key endogenous variables that shape TFP. Without assuming a linear, perfectly competitive and frictionless outside sector to equalize wages across countries, we show how to solve the model with the help of a simple scissors diagram.

A central object of interest, besides the focus on TFP, is the HME which has been used as a criterion to discriminate between trade models featuring increasing returns to scale and 
more conventional comparative advantage based setups. We show that models with selection feature a HME driven by selection together with the more traditional wage-based HME. It is magnified by falling trade costs and translates into cross-country differences in average productivity. These determine TFP together with external economies of scale. In contrast to the model with a linear outside sector, trade liberalization attenuates these cross-country differences and leads to real wage convergence. Firm-level heterogeneity is absolutely crucial for these results: in the Krugman (1980) single-sector model, no HME can arise.

Our results matter for the empirical HME literature since they suggest that failing to control for the level of technology may wrongly reject the existence of an endowment-driven HME. Moreover, our results also matter for quantitative trade theory models based on the Krugman (1980) or the Melitz (2003) models. They suggest that the standard specification of the Dixit-Stiglitz aggregate production function implies too high a degree of external economies of scale (EoS) to square the data. Lower EoS have obvious effects on the size of gains from trade in quantitative trade models and on optimal policies. When EoS differ from the degree implied by the standard CES aggregate, welfare calculus à la Arkolakis et al. (2012) requires knowledge about an additional endogenous variable, namely the mass of inputs, and an additional parameter, namely $\mu /(\sigma-1)$. 


\section{References}

[1] Alcalá, F. and A. Ciccone (2004), 'Trade and Productivity', Quarterly Journal of Economics 119(2): 613-646.

[2] Ardelean, A. (2007), 'How strong is love of variety?', mimeo: Purdue University.

[3] Arkolakis, C., N. Ramondo, A. Rodriguez-Clare, and S. Yeaple (2013), 'Innovation and Production in the Global Economy', NBER Working Paper No. 18972.

[4] Baldwin, R. E. and R. Forslid (2010), 'Trade Liberalization with Heterogeneous Firms', Review of Development Economics 14(2): 161-176.

[5] Baldwin, R. E. and T. Okubo (2009), 'Tax Reform Delocation, and Heterogeneous Firms', Scandinavian Journal of Economics 111 (4): 741-764.

[6] Balistreri, E. J., R. H. Hillberry, and T. F. Rutherford (2011), 'Structural Estimation and Solution of International Trade Models with Heterogeneous Firms', Journal of International Economics 83: 98-108.

[7] Basu, S., L. Pascali, F. Schiantarelli, and L. Serven (2012), 'Productivity and the Welfare of Nations', NBER Working Paper No. 17971.

[8] Benassy, J.-P. (1998), 'Is There Always too Little Research in Endogenous Growth with Expanding Product Variety?', European Economic Review 42: 61-69.

[9] Bernard, A., J. Eaton, B. Jensen, and S. Kortum (2003), 'Plants and Productivity in International Trade', American Economic Review 93(4): 1268-1290.

[10] Bernard, A., B. Jensen, S. Redding, and P. Schott (2007), 'Firms in International Trade', Journal of Economic Perspectives, Summer 2007.

[11] Blanchard, O. and F. Giavazzi (2003), 'Macroeconomic Effects of Regulation and Deregulation in Goods and Factor Markets', Quarterly Journal of Economics 118: 879-907.

[12] Costinot, A. and A. Rodriguez-Clare (2015), 'Trade Theory with Numbers: Quantifying the Consequences of Globalization', in G. Gopinath, E. Helpman and K. Rogoff (eds.): Handbook of International Economics, Vol. 4., 197-261. 
[13] Chor, D. (2009), 'Subsidies for FDI: Implications from a Model with Heterogeneous Firms', Journal of International Economics 78: 113-125.

[14] Crozet, M. and F. Trionfetti (2008), 'Trade Costs and the Home Market Effect', Journal of International Economics 76: 309-321.

[15] Davis, D. R. (1998), 'The Home Market, Trade, and Industrial Structure', American Economic Review 88(5): 1264-1276.

[16] Davis, D. R. and D. E. Weinstein (1999), 'Economic Geography and Regional Production Structure; An Empirical Investigation', European Economic Review 43: 379-407.

[17] Davis, D. R. and D. E. Weinstein (2003), 'Market Access, Economic Geography and Comparative Advantage: An Empirical Test', Journal of International Economics 59(1): 1-23.

[18] Demidova, S. (2008), 'Productivity Improvements and Falling Trade Costs: Boon or Bane?', International Economic Review 49(4): 1437-1462.

[19] Demidova, S. and A. Rodriguez-Clare (2013), 'The Simple Analytics of the Melitz Model in a Small Open Economy', Journal of International Economics 90(2): 266-272.

[20] Dixit, A. K. and J. E. Stiglitz (1977), 'Monopolistic Competition and Optimum Product Diversity', American Economic Review 67(3): 297-308.

[21] Feenstra, R. C., J. R. Markusen, and A. K. Rose (2001), 'Using the Gravity Equation to Differentiate among Alternative Theories of Trade', Canadian Journal of Economics 34(2): 430-447.

[22] Feenstra, R. C., R. Inklaar, and M. P. Timmer (2015), 'The Next Generation of the Penn World Table', American Economic Review, forthcoming.

[23] Felbermayr, G. and J. Gröschl (2013), 'Natural Disasters and the Effect of Trade on Income: A New Panel IV Approach.' European Economic Review 58: 18-30.

[24] Frankel, J. A. and D. H. Romer (1999), 'Does Trade Cause Growth?', American Economic Review 89(3): 379-399. 
[25] Grossman, G. M., E. Helpman, and A. Szeidl (2006), 'Optimal Integration Strategies for the Multinational Firm', Journal of International Economics 70(1): 216-238.

[26] Hanson, G. H. and C. Xiang (2004), 'The Home Market Effect and Bilateral Trade Patterns', American Economic Review 94(4): 1108-1129.

[27] Head, K. and J. Ries (2001), 'Increasing Returns versus National Product Differentiation as an Explanation for the Pattern of US-Canada Trade', American Economic Review 91 (4): 858-876.

[28] Head, K. and T. Mayer (2004), 'The Empirics of Agglomeration and Trade', in J. V. Henderson and J.-F. Thisse (eds.), Handbook of Regional and Urban Economics, Vol. 4 (Amsterdam: New Holland): 2609-2669.

[29] Helpman, E. and P. Krugman (1985), 'Market Structure and Foreign Trade: Increasing Returns, Imperfect Competition, and the International Economy' (Cambridge, MA: MIT Press).

[30] Helpman, E., M. Melitz, and S. Yeaple (2004), 'Export versus FDI with Heterogeneous Firms', American Economic Review 94(1): 300-316.

[31] Irwin, D. A. and M. Terviö (2002), 'Does Trade Raise Income?: Evidence from the Twentieth Century', Journal of International Economics 58(1): 1-18.

[32] Jones, C. (1995), 'Time Series Tests of Endogenous Growth Models', Quarterly Journal of Economics 110(2): 495-525.

[33] Krugman, P. (1980), 'Scale Economies, Product Differentiation, and the Pattern of Trade', American Economic Review 70(5): 950-959.

[34] Melitz, M. J. (2003), 'The Impact of Trade on Intra-Industry Reallocations and Aggregate Industry Productivity', Econometrica 71: 1695-1725.

[35] Melitz, M. J. and G. I. P. Ottaviano (2008), 'Market Size, Trade, and Productivity', Review of Economic Studies 75(1): 295-316. 
[36] Melitz, M. J. and S. Redding (2014), 'Heterogeneous Firms and Trade', in: Gopinath, G., Helpman, E., and Rogoff, K., Handbook of International Economics Vol 4, Ch. 1, 1-54, Elsevier (Amsterdam)

[37] Melitz, M. J. and D. Trefler (2012), 'Gains From Trade When Firms Matter', Journal of Economic Perspectives 26(2):91-118.

[38] Ossa, R. (2011), 'A “New Trade” Theory of GATT/WTO Negotiations', Journal of Political Economy 119(1): 122-152.

[39] Ramondo, N., A. Rodriguez-Clare, and M. Saborio-Rodriguez (2015), 'Trade, Domestic Frictions, and Scale Effects', mimeo: UC-San Diego.

[40] Romer, P. (1990), 'Endogenous Technological Change', Journal of Political Economy 98: S71-S102.

[41] Rose, A. (2006), 'Size Really Doesn't Matter: In Search of a National Scale Effect', Journal of the International and Japanese Economies 20: 482-507.

[42] Segerstrom, P. S. and Y. Sugita (2015), 'A New Way of Solving the Melitz Model Using Simple and Intuitive Diagrams', mimeo: Stockholm School of Economics. 


\section{A Stylized Facts: Robustness Checks}

Figure 8: Scale and welfare across countries

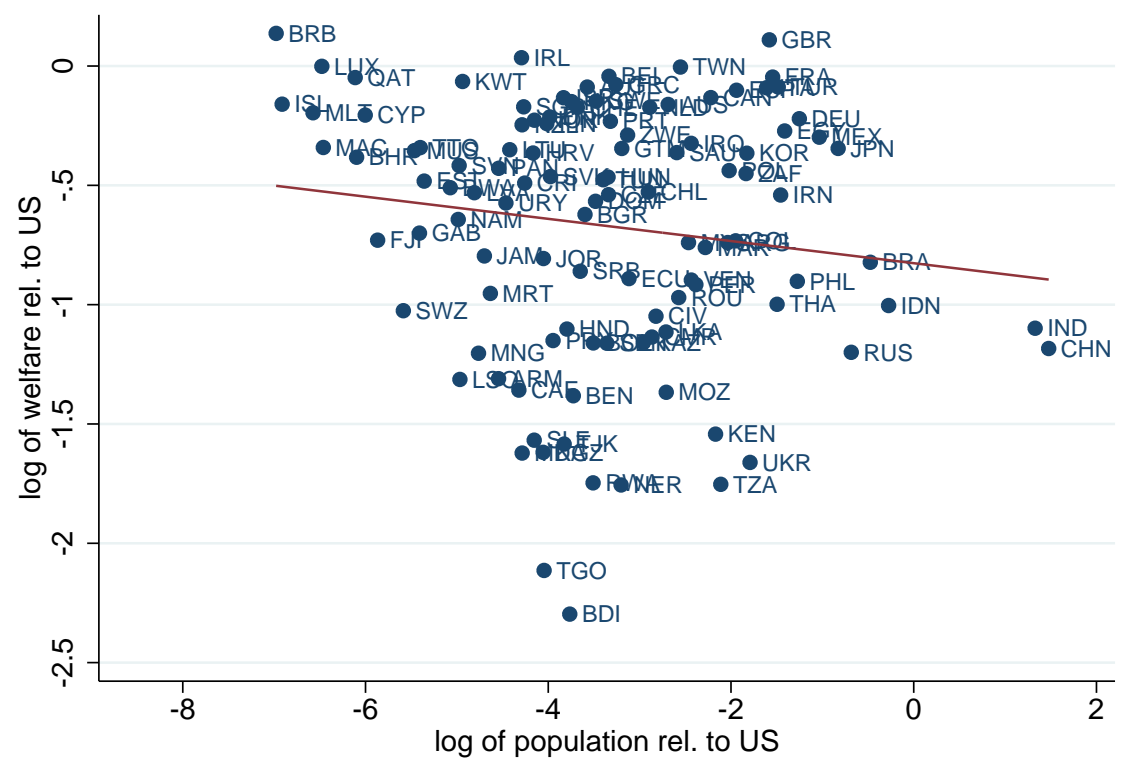

Notes: Data from Penn World Tables 8.1 (Feenstra et al., 2015); series cwtfp (welfare-relevant TFP levels at current PPPs) and pop, both relative to the respective US values. Linear univariate regression on averages for year 20012005, coefficient: -0.046 , robust t-value: $-1.87 . N=110$. 
Table 2: Robustness Analysis: Size-welfare correlations in cross-sections

\begin{tabular}{|c|c|c|c|c|c|c|}
\hline & (1) & $(2)$ & (3) & (4) & (5) & (6) \\
\hline De & var.: $\log$ of & welfare-re & evant TFP 1 & vels at curr & nt PPPs & \\
\hline & & 1995-99 & & & 2005-09 & \\
\hline Log population & -0.0406 & $-0.0609^{* *}$ & -0.00143 & $-0.0453^{* *}$ & $-0.0665^{* * *}$ & -0.0257 \\
\hline & $(0.0251)$ & $(0.0236)$ & $(0.0347)$ & $(0.0209)$ & $(0.0194)$ & $(0.0292)$ \\
\hline Landlocked $(0,1)$ & & $-0.420 * * *$ & $-0.378 * * *$ & & $-0.425^{* * *}$ & $-0.396 * * *$ \\
\hline & & $(0.138)$ & $(0.144)$ & & $(0.122)$ & $(0.127)$ \\
\hline Log area & & & $-0.0567^{* *}$ & & & $-0.0383^{*}$ \\
\hline & & & $(0.0236)$ & & & $(0.0199)$ \\
\hline Constant & $-0.762^{* * *}$ & $-0.751^{* * *}$ & 0.118 & $-0.733^{* * *}$ & $-0.724^{* * *}$ & -0.136 \\
\hline & $(0.100)$ & $(0.0978)$ & $(0.373)$ & $(0.0811)$ & $(0.0771)$ & $(0.316)$ \\
\hline R-squared & 0.016 & 0.117 & 0.147 & 0.025 & 0.150 & 0.167 \\
\hline & (7) & (8) & (9) & (10) & (11) & (12) \\
\hline & Dep.v & ar.: $\log$ of $\mathrm{T}$ & $P$ level at c & Irrent PPPs & & \\
\hline & & 1995-99 & & & 2005-09 & \\
\hline Log population & -0.0432 & $-0.0667^{* *}$ & -0.00512 & $-0.0457^{*}$ & $-0.0693^{* * *}$ & -0.0147 \\
\hline & $(0.0275)$ & $(0.0257)$ & $(0.0378)$ & $(0.0255)$ & $(0.0246)$ & $(0.0346)$ \\
\hline Landlocked $(0,1)$ & & $-0.486^{* * *}$ & $-0.442^{* * *}$ & & $-0.471^{* * *}$ & $-0.432^{* * *}$ \\
\hline & & $(0.149)$ & $(0.156)$ & & $(0.139)$ & $(0.145)$ \\
\hline Log area & & & $-0.0587^{* *}$ & & & $-0.0512^{* *}$ \\
\hline & & & $(0.0261)$ & & & $(0.0251)$ \\
\hline Constant & $-0.762^{* * *}$ & $-0.750^{* * *}$ & 0.150 & $-0.676^{* * *}$ & $-0.666^{* * *}$ & 0.120 \\
\hline & $(0.105)$ & $(0.102)$ & $(0.410)$ & $(0.0890)$ & $(0.0851)$ & $(0.387)$ \\
\hline R-squared & 0.016 & 0.129 & 0.157 & 0.018 & 0.132 & 0.154 \\
\hline
\end{tabular}

Notes: Robust standard errors in parentheses, ${ }^{* * *} \mathrm{p}<0.01,{ }^{* *} \mathrm{p}<0.05,{ }^{*} \mathrm{p}<0.1$. Number of observations: 110 in all regressions. Note: larger sample than in Figure 1. 
Table 3: Robustness Analysis: Size-welfare correlations in panel regressions

\begin{tabular}{|c|c|c|c|c|c|c|c|c|}
\hline \multirow{4}{*}{$\begin{array}{l}\text { Sample: } \\
\text { Indep. var.: } \\
\text { Method: }\end{array}$} & (1) & (2) & (3) & (4) & (5) & (6) & (7) & (8) \\
\hline & \multicolumn{4}{|c|}{ unbalanced } & \multicolumn{4}{|c|}{ balanced } \\
\hline & \multicolumn{2}{|c|}{ POP } & \multicolumn{2}{|c|}{ EMP } & \multicolumn{2}{|c|}{ POP } & \multicolumn{2}{|c|}{ EMP } \\
\hline & FD & FE & FD & FE & FD & FE & FD & FE \\
\hline $\begin{array}{l}\text { Dep.var. } \\
\text { cwtfp }\end{array}$ & $\begin{array}{c}-0.320 \\
(0.0756)\end{array}$ & $\begin{array}{c}-0.519 \\
(0.0780)\end{array}$ & $\begin{array}{c}-0.198 \\
(0.0609)\end{array}$ & $\begin{array}{c}-0.408 \\
(0.0609)\end{array}$ & $\begin{array}{l}-0.345 \\
(0.116)\end{array}$ & $\begin{array}{l}-0.453 \\
(0.103)\end{array}$ & $\begin{array}{c}-0.236 \\
(0.0997)\end{array}$ & $\begin{array}{c}-0.362 \\
(0.0894)\end{array}$ \\
\hline rwtfpna & $\begin{array}{c}-0.243 \\
(0.0781)\end{array}$ & $\begin{array}{c}-0.400 \\
(0.0768)\end{array}$ & $\begin{array}{c}-0.154 \\
(0.0564)\end{array}$ & $\begin{array}{c}-0.312 \\
(0.0531)\end{array}$ & $\begin{array}{l}-0.333 \\
(0.103)\end{array}$ & $\begin{array}{c}-0.422 \\
(0.0932)\end{array}$ & $\begin{array}{c}-0.254 \\
(0.0772)\end{array}$ & $\begin{array}{c}-0.335 \\
(0.0682)\end{array}$ \\
\hline $\operatorname{ctfp}$ & $\begin{array}{c}-0.490 \\
(0.0989)\end{array}$ & $\begin{array}{c}-0.627 \\
(0.0887)\end{array}$ & $\begin{array}{c}-0.276 \\
(0.0722)\end{array}$ & $\begin{array}{c}-0.467 \\
(0.0680)\end{array}$ & $\begin{array}{l}-0.501 \\
(0.148)\end{array}$ & $\begin{array}{l}-0.564 \\
(0.111)\end{array}$ & $\begin{array}{c}-0.256 \\
(0.0947)\end{array}$ & $\begin{array}{c}-0.405 \\
(0.0916)\end{array}$ \\
\hline rtfpna & $\begin{array}{c}-0.424 \\
(0.0840)\end{array}$ & $\begin{array}{c}-0.515 \\
(0.0844)\end{array}$ & $\begin{array}{c}-0.277 \\
(0.0614)\end{array}$ & $\begin{array}{c}-0.385 \\
(0.0658)\end{array}$ & $\begin{array}{l}-0.398 \\
(0.102)\end{array}$ & $\begin{array}{c}-0.510 \\
(0.0916)\end{array}$ & $\begin{array}{c}-0.269 \\
(0.0647)\end{array}$ & $\begin{array}{c}-0.362 \\
(0.0703)\end{array}$ \\
\hline rgdpe/pop & $\begin{array}{l}-0.390 \\
(0.109)\end{array}$ & $\begin{array}{l}-0.793 \\
(0.116)\end{array}$ & $\begin{array}{c}0.0149 \\
(0.0881)\end{array}$ & $\begin{array}{c}-0.335 \\
(0.0959)\end{array}$ & $\begin{array}{l}-0.537 \\
(0.136)\end{array}$ & $\begin{array}{l}-0.628 \\
(0.154)\end{array}$ & $\begin{array}{c}-0.00406 \\
(0.130)\end{array}$ & $\begin{array}{l}-0.241 \\
(0.151)\end{array}$ \\
\hline rgdpo/pop & $\begin{array}{l}-0.480 \\
(0.122)\end{array}$ & $\begin{array}{l}-0.843 \\
(0.116)\end{array}$ & $\begin{array}{l}-0.0342 \\
(0.0984)\end{array}$ & $\begin{array}{c}-0.423 \\
(0.0921)\end{array}$ & $\begin{array}{l}-0.697 \\
(0.153)\end{array}$ & $\begin{array}{l}-0.734 \\
(0.150)\end{array}$ & $\begin{array}{c}-0.0595 \\
(0.123)\end{array}$ & $\begin{array}{l}-0.326 \\
(0.142)\end{array}$ \\
\hline cgdpe/pop & $\begin{array}{l}-0.389 \\
(0.108)\end{array}$ & $\begin{array}{l}-0.775 \\
(0.114)\end{array}$ & $\begin{array}{c}0.0122 \\
(0.0882)\end{array}$ & $\begin{array}{c}-0.329 \\
(0.0947)\end{array}$ & $\begin{array}{l}-0.517 \\
(0.132)\end{array}$ & $\begin{array}{l}-0.598 \\
(0.151)\end{array}$ & $\begin{array}{c}0.00451 \\
(0.129)\end{array}$ & $\begin{array}{l}-0.225 \\
(0.148)\end{array}$ \\
\hline cgdpo/pop & $\begin{array}{l}-0.446 \\
(0.118)\end{array}$ & $\begin{array}{l}-0.776 \\
(0.116)\end{array}$ & $\begin{array}{l}-0.0256 \\
(0.0894)\end{array}$ & $\begin{array}{c}-0.362 \\
(0.0927)\end{array}$ & $\begin{array}{l}-0.651 \\
(0.168)\end{array}$ & $\begin{array}{l}-0.669 \\
(0.154)\end{array}$ & $\begin{array}{c}-0.0292 \\
(0.122)\end{array}$ & $\begin{array}{l}-0.276 \\
(0.146)\end{array}$ \\
\hline rgdpe/emp & $\begin{array}{l}-0.433 \\
(0.115)\end{array}$ & $\begin{array}{l}-0.806 \\
(0.113)\end{array}$ & $\begin{array}{c}-0.403 \\
(0.0778)\end{array}$ & $\begin{array}{c}-0.598 \\
(0.0873)\end{array}$ & $\begin{array}{l}-0.555 \\
(0.138)\end{array}$ & $\begin{array}{l}-0.684 \\
(0.146)\end{array}$ & $\begin{array}{l}-0.491 \\
(0.109)\end{array}$ & $\begin{array}{l}-0.512 \\
(0.134)\end{array}$ \\
\hline rgdpo/emp & $\begin{array}{l}-0.548 \\
(0.133)\end{array}$ & $\begin{array}{l}-0.876 \\
(0.116)\end{array}$ & $\begin{array}{c}-0.452 \\
(0.0897)\end{array}$ & $\begin{array}{c}-0.686 \\
(0.0853)\end{array}$ & $\begin{array}{l}-0.715 \\
(0.153)\end{array}$ & $\begin{array}{l}-0.790 \\
(0.146)\end{array}$ & $\begin{array}{l}-0.547 \\
(0.103)\end{array}$ & $\begin{array}{l}-0.597 \\
(0.125)\end{array}$ \\
\hline cgdpe/emp & $\begin{array}{l}-0.430 \\
(0.114)\end{array}$ & $\begin{array}{l}-0.788 \\
(0.112)\end{array}$ & $\begin{array}{c}-0.406 \\
(0.0775)\end{array}$ & $\begin{array}{c}-0.592 \\
(0.0863)\end{array}$ & $\begin{array}{l}-0.535 \\
(0.135)\end{array}$ & $\begin{array}{l}-0.654 \\
(0.144)\end{array}$ & $\begin{array}{l}-0.482 \\
(0.108)\end{array}$ & $\begin{array}{l}-0.496 \\
(0.132)\end{array}$ \\
\hline cgdpo/emp & $\begin{array}{l}-0.499 \\
(0.128)\end{array}$ & $\begin{array}{l}-0.800 \\
(0.117)\end{array}$ & $\begin{array}{c}-0.441 \\
(0.0801)\end{array}$ & $\begin{array}{c}-0.624 \\
(0.0866)\end{array}$ & $\begin{array}{l}-0.668 \\
(0.165)\end{array}$ & $\begin{array}{l}-0.726 \\
(0.149)\end{array}$ & $\begin{array}{l}-0.516 \\
(0.103)\end{array}$ & $\begin{array}{l}-0.547 \\
(0.130)\end{array}$ \\
\hline
\end{tabular}

Notes: Each cell corresponds to a separate regression, with all variables in logs. The vast majority of coefficients is statistically significant at the $1 \%$ level. All models contain full sets of period fixed effects. Robust standard errors (clustered at country level) in paranthesis. PWT 8.1. variables. cwtfp: welfare-relevant TFP levels at current PPPs, rwtf pna: welfare-relevant TFP at constant national prices $(2005=1)$, ctfp: TFP level at current PPPs, rtfpna: TFP at constant national prices (2005=1); rgdpe: expenditure-side real GDP at chained PPPs; rgdpo: output-side real GDP at chained PPPs; cgdpe: expenditure-side real GDP at current PPPs; cgdpo: output-side real GDP at current PPPs. Balanced sample always contains 68 countries, unabalanced sample contains 110 countries when dep.var. is a TFP variable, and 165 countries whendep.var. is a GDP variable. 


\section{B Additional simulation results}

Figure 9: Threshold external economies of scale $\bar{\mu}$ and the population share

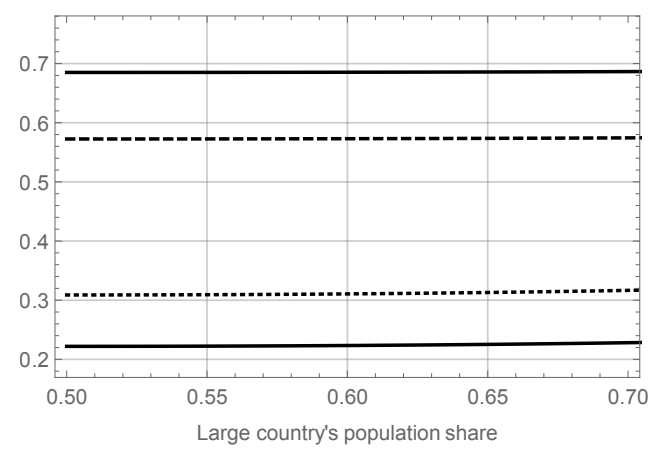

Notes: We plot the degree of external economies of scale $\bar{\mu}$ that makes relative TFP independent of relative country size on the $y$-axis and the large country's population share on the $x$-axis. We show $\bar{\mu}$ for (i) the benchmark parametrization (solid line), (ii) a trade liberalization scenario $\tau=1$ (dashed line), (iii) a productivity dispersion increase $\beta=3.925$, a value taken from Balistreri et al. (2011), (dotted line), and (iv) trade liberalization $\tau=1$ and increase in productivity dispersion $(\beta=3.952)$ at the same time. In scenarios (ii)-(iv), all other parameters are set as in the benchmark parametrization. 


\section{Proofs of Propositions}

\section{C.1 Proof of Proposition 3 (Home Market Magnification Effect)}

In order to prove that higher productivity dispersion (lower $\beta$ ) results in a lower relative entry probability $\chi$, we show that both the ex post and ex ante profitability curve rotate upwards. Figure 5 then implies that the equilibrium $\chi$ in the new equilibrium must be larger than in the initial equilibrium.

Ex post profitability. Evaluated at $\omega=1$, the ex post profitability curve yields $\chi=L$ independently of $\beta$. Moreover, we have

$$
\frac{\partial \chi}{\partial \beta}=-\frac{2}{\rho} L \omega^{-\frac{2 \beta-\rho}{\rho}} \ln [\omega]<0,
$$

where the inequality follows from $\omega>1$. Hence, an increase in productivity dispersion (lower $\beta$ ) leads to an upward rotation of the ex post profitability curve.

Ex ante profitability. Evaluated at $\omega=1$, the ex ante profitability curve yields $\chi=1$ independently of $\beta$. Moreover, we have

$$
\frac{\partial \chi}{\partial \beta}=\frac{\eta}{\rho} \frac{\omega^{\frac{\beta}{\rho}}+\omega^{-\frac{\beta}{\rho}}-2 \eta}{\left(1-\eta \omega^{\frac{\beta}{\rho}}\right)^{2}} \ln [\omega]+\frac{\omega^{\frac{\beta}{\rho}}-\omega^{-\frac{\beta}{\rho}}}{\left(1-\eta \omega^{\frac{\beta}{\rho}}\right)^{2}} \frac{\partial \eta}{\partial \beta},
$$

where the first term is positive since $\omega^{\frac{\beta}{\rho}}-\eta>0, \omega^{-\frac{\beta}{\rho}}-\eta>0$, and $\omega>1$. The second term is negative since

$$
\frac{\partial \eta}{\partial \beta}=-\eta\left(\ln [\tau]+\frac{1}{\sigma-1} \ln \left[\frac{f^{x}}{f^{d}}\right]\right)<0
$$

as $\tau \geq 1$ and $f^{x} / f^{d}>1$.

The sign of $\partial \chi / \partial \beta$ is then given by the sign of

$$
\left(\omega^{\frac{\beta}{\rho}}+\omega^{-\frac{\beta}{\rho}}-2 \eta\right) \frac{\ln [\omega]}{\rho}-\left(\omega^{\frac{\beta}{\rho}}-\omega^{-\frac{\beta}{\rho}}\right)\left(\ln [\tau]+\frac{1}{\sigma-1} \ln \left[\frac{f^{x}}{f^{d}}\right]\right) .
$$


We conjecture $\partial \chi / \partial \beta<0$, which requires

$$
\left(\omega^{\frac{\beta}{\rho}}+\omega^{-\frac{\beta}{\rho}}-2 \eta\right) \frac{\ln [\omega]}{\rho}<\left(\omega^{\frac{\beta}{\rho}}-\omega^{-\frac{\beta}{\rho}}\right)\left(\ln [\tau]+\frac{1}{\sigma-1} \ln \left[\frac{f^{x}}{f^{d}}\right]\right) .
$$

Multiplying both sides by $\beta$, we obtain

$$
\left(\omega^{\frac{\beta}{\rho}}+\omega^{-\frac{\beta}{\rho}}-2 \eta\right) \frac{\beta \ln [\omega]}{\rho}<\left(\omega^{\frac{\beta}{\rho}}-\omega^{-\frac{\beta}{\rho}}\right)\left(\beta \ln [\tau]+\frac{\beta}{\sigma-1} \ln \left[\frac{f^{x}}{f^{d}}\right]\right)
$$

Adding a virtual zero on the right hand side and employing the definition of $\eta \equiv \tau^{-\beta}\left(\frac{f^{x}}{f^{d}}\right)^{1-\frac{\beta}{\sigma-1}}$, we obtain

$$
\begin{aligned}
& \left(\omega^{\frac{\beta}{\rho}}+\omega^{-\frac{\beta}{\rho}}-2 \eta\right) \frac{\beta \ln [\omega]}{\rho}<\left(\omega^{\frac{\beta}{\rho}}-\omega^{-\frac{\beta}{\rho}}\right)\left(\beta \ln [\tau]+\left(\frac{\beta}{\sigma-1}-1\right) \ln \left[\frac{f^{x}}{f^{d}}\right]+\ln \left[\frac{f^{x}}{f^{d}}\right]\right) \Leftrightarrow \\
& \left(\omega^{\frac{\beta}{\rho}}+\omega^{-\frac{\beta}{\rho}}-2 \eta\right) \frac{\beta \ln [\omega]}{\rho}<\left(\omega^{\frac{\beta}{\rho}}-\omega^{-\frac{\beta}{\rho}}\right)\left(-\ln [\eta]+\ln \left[\frac{f^{x}}{f^{d}}\right]\right) .
\end{aligned}
$$

A sufficient condition for this inequality to hold is

$$
\left(\omega^{\frac{\beta}{\rho}}+\omega^{-\frac{\beta}{\rho}}-2 \eta\right) \frac{\beta \ln [\omega]}{\rho}<-\left(\omega^{\frac{\beta}{\rho}}-\omega^{-\frac{\beta}{\rho}}\right) \ln [\eta]
$$

as $f^{x}>f^{d}$ implies $\ln \left[\frac{f^{x}}{f^{d}}\right]>0$.

Let $x \equiv \omega^{\frac{\beta}{\rho}}$. Then, the above inequality can be rewritten as

$$
\begin{aligned}
\left(x+x^{-1}-2 \eta\right) \frac{\beta \ln \left[x^{\frac{\rho}{\beta}}\right]}{\rho} & <-\left(x-x^{-1}\right) \ln [\eta] \Leftrightarrow \\
\left(x+x^{-1}-2 \eta\right) \ln [x] & <-\left(x-x^{-1}\right) \ln [\eta] .
\end{aligned}
$$

We now check whether the above inequality holds for all possible combinations of $\eta \in(0,1)$ and $x \in\left(1, \eta^{-1}\right)$. Notice that it not required to set parameters of the model in order to check the inequality. Numerical simulation shows that the inequality holds for all possible combinations. Hence, the ex ante profitability curve rotates upwards in response to an increase in productivity dispersion (lower $\beta$ ). 
New equilibrium. Given that both curves rotate upwards, the equilibrium value of $\chi$ must rise in response to an drop in $\beta$ (higher dispersion). According to proposition 2, a rise in $\chi$ leads to a rise in the relative mass of active firms. Hence, productivity dispersion magnifies the effect of relative country size on the relative mass of active firms, which proves part (b) of proposition 3.

\section{C.2 Proof of Proposition 4 (Available inputs)}

According to equation (12), we have

$$
\frac{N}{L}=\chi \frac{\frac{f^{x}}{f^{d}}+\eta \omega^{\frac{\beta}{\rho}} L^{-1}}{\frac{f^{x}}{f^{d}}+\eta \omega^{-\frac{\beta}{\rho}} L} .
$$

Starting from the free entry conditions, employing the zero cutoff profit conditions in relative terms, and using the definition $\chi=\left(\varphi_{F F}^{*} / \varphi_{H H}^{*}\right)^{\beta}$, we obtain

$$
\chi=\frac{1+\eta \omega^{\frac{\beta}{\rho}} \chi}{1+\eta \omega^{-\frac{\beta}{\rho}} \chi^{-1}} .
$$

Employing the ex post profitability curve to substitute out the $\chi$ terms on the right hand side of this expression, we get

$$
\chi=\frac{1+\eta L \omega^{-\frac{\beta-\rho}{\rho}}}{1+\eta L^{-1} \omega^{\frac{\beta-\rho}{\rho}}} .
$$

Using this expression to substitute out the $\chi$ term from equation (), we obtain

$$
\begin{aligned}
\frac{N}{L} & =\frac{1+\eta L \omega^{-\frac{\beta-\rho}{\rho}} \frac{f^{x}}{f^{d}}+\eta \omega^{\frac{\beta}{\rho}} L^{-1}}{1+\eta L^{-1} \omega^{\frac{\beta-\rho}{\rho}} \frac{f^{x}}{f^{d}}+\eta \omega^{-\frac{\beta}{\rho}} L} \\
& =\frac{\frac{f^{x}}{f^{d}}+\eta \omega^{\frac{\beta}{\rho}} L^{-1}+\eta L \omega^{-\frac{\beta-\rho}{\rho}} \frac{f^{x}}{f^{d}}+\eta L \omega^{-\frac{\beta-\rho}{\rho}} \eta \omega^{\frac{\beta}{\rho}} L^{-1}}{\frac{f^{x}}{f^{d}}+\eta \omega^{-\frac{\beta}{\rho}} L+\frac{f^{x}}{f^{d}} \eta L^{-1} \omega^{\frac{\beta-\rho}{\rho}}+\eta L^{-1} \omega^{\frac{\beta-\rho}{\rho}} \eta \omega^{-\frac{\beta}{\rho}} L} \\
& =\frac{\frac{f^{x}}{f^{d}}+\eta \omega^{\frac{\beta}{\rho}} L^{-1}+\eta L \omega^{-\frac{\beta-\rho}{\rho}} \frac{f^{x}}{f^{d}}+\eta^{2} \omega}{\frac{f^{x}}{f^{d}}+\eta \omega^{-\frac{\beta}{\rho}} L+\frac{f^{x}}{f^{d}} \eta L^{-1} \omega^{\frac{\beta-\rho}{\rho}}+\eta^{2} \omega^{-1}} .
\end{aligned}
$$


$N / L>1$ then requires

$$
\begin{aligned}
\frac{f^{x}}{f^{d}}+\eta \omega^{\frac{\beta}{\rho}} L^{-1}+\eta L \omega^{-\frac{\beta-\rho}{\rho}} \frac{f^{x}}{f^{d}}+\eta^{2} \omega & >1 \\
\frac{f^{x}}{f^{d}}+\eta \omega^{-\frac{\beta}{\rho}} L+\frac{f^{x}}{f^{d}} \eta L^{-1} \omega^{\frac{\beta-\rho}{\rho}}+\eta^{2} \omega^{-1} & \\
\frac{f^{x}}{f^{d}}+\eta \omega^{\frac{\beta}{\rho}} L^{-1}+\eta L \omega^{-\frac{\beta-\rho}{\rho}} \frac{f^{x}}{f^{d}}+\eta^{2} \omega & >\frac{f^{x}}{f^{d}}+\eta \omega^{-\frac{\beta}{\rho}} L+\frac{f^{x}}{f^{d}} \eta L^{-1} \omega^{\frac{\beta-\rho}{\rho}}+\eta^{2} \omega^{-1} \Leftrightarrow \\
\eta\left(\omega-\omega^{-1}\right)+\omega^{-\frac{\beta}{\rho}} L\left(\omega \frac{f^{x}}{f^{d}}-1\right)-L^{-1} \omega^{\frac{\beta-\rho}{\rho}}\left(\frac{f^{x}}{f^{d}}-\omega\right) & >0 .
\end{aligned}
$$

In this expression, $\eta\left(\omega-\omega^{-1}\right)>0$ as $\omega>1$. A sufficient condition for the inequality to hold is then

$$
\begin{aligned}
& \omega^{-\frac{\beta}{\rho}} L\left(\omega \frac{f^{x}}{f^{d}}-1\right)-L^{-1} \omega^{\frac{\beta-\rho}{\rho}}\left(\frac{f^{x}}{f^{d}}-\omega\right)>0 \Leftrightarrow \\
& \omega^{-\frac{\beta-\rho}{\rho}} L^{2}\left(\frac{f^{x}}{f^{d}}-\omega^{-1}\right)>\omega^{\frac{\beta-\rho}{\rho}}\left(\frac{f^{x}}{f^{d}}-\omega\right) \\
& \omega^{-2 \frac{\beta-\rho}{\rho}} L^{2}\left(\frac{f^{x}}{f^{d}}-\omega^{-1}\right)>\frac{f^{x}}{f^{d}}-\omega .
\end{aligned}
$$

Consider the case $f^{x} \geq f^{d}$. As $\omega>1$ and $\omega \leq \eta^{-\frac{\rho}{\beta}}<\left(\frac{f^{x}}{f^{d}}\right)^{\frac{\beta-(\sigma-1)}{\beta \sigma}}<\frac{f^{x}}{f^{d}}$, we have $\frac{f^{x}}{f^{d}}-\omega^{-1}>$ $\frac{f^{x}}{f^{d}}-\omega$. A sufficient condition for the inequality to hold is

$$
\omega^{-2 \frac{\beta-\rho}{\rho}} L^{2}>1 \Leftrightarrow L>\omega^{\frac{\beta-\rho}{\rho}} \Leftrightarrow L>\omega^{\frac{\beta-\rho}{\rho}} \Leftrightarrow \omega<L^{\frac{\rho}{\beta-\rho}}
$$

which means that the equilibrium wage rate must be sufficiently small.

From our analysis above, we know that the equilibrium wage is bounded from above by

$$
\chi=L \omega^{-\frac{2 \beta-\rho}{\rho}}>1 \Leftrightarrow \omega<L^{\frac{\rho}{2 \beta-\rho}} .
$$

This requirement is stronger than $\omega<L^{\frac{\rho}{\beta-\rho}}$ as

$$
L^{\frac{\rho}{2 \beta-\rho}}<L^{\frac{\rho}{\beta-\rho}} \Leftrightarrow \frac{\rho}{2 \beta-\rho}<\frac{\rho}{\beta-\rho} \Leftrightarrow \beta-\rho<2 \beta-\rho,
$$

which clearly holds. Hence, we have established that $N / L>1$. 


\section{Supplementary Material for “Market Size Effects in New New Trade Theory"}

(Not for Publication)

In this supplementary material, we show the derivations in more detail (part D.1), analyze the case where the sorting condition does not hold (part D.2), and consider a set out a version of the Melitz model with an outside sector (D.3).

\section{D.1 Detailed derivations}

Final good producer. The final good producer chooses the profit-maximizing input quantities $q_{i}[z]$, taking the price of final good $P_{i}$, prices of inputs $p_{i}[z]$, and the mass of available inputs $N_{i}$ as given:

$$
\max _{q_{i}[z]} P_{i}\left[N_{i}^{\frac{\mu-1}{\sigma}} \int_{z \in \Omega_{i}} q_{i}[z]^{\frac{\sigma-1}{\sigma}}\right]^{\frac{\sigma}{\sigma-1}}-\int_{z \in \Omega_{i}} p_{i}[z] q_{i}[z]
$$

The first-order condition of the profit-maximization problem is

$$
P_{i} N_{i}^{\frac{\mu-1}{\sigma-1}} q_{i}[z]^{-\frac{1}{\sigma}}\left[\int_{z \in \Omega_{i}} q_{i}[z]^{\frac{\sigma-1}{\sigma}}\right]^{\frac{\sigma}{\sigma-1}-1}=p_{i}[z]
$$

Taking the ratio of the first-order conditions for any two inputs $z_{1}$ and $z_{2}$, we obtain

$$
\frac{q\left[z_{1}\right]}{q\left[z_{2}\right]}=\left(\frac{p_{i}\left[z_{1}\right]}{p_{i}\left[z_{2}\right]}\right)^{-\sigma}
$$

Using this expression in the cost (expenditure) function, we obtain

$$
E_{i}=q_{i}[z] p_{i}[z]^{\sigma} \int_{z \in \Omega_{i}} p_{i}[z]^{1-\sigma} \Leftrightarrow q_{i}[z]=\frac{E_{i} p_{i}[z]^{-\sigma}}{\int_{z \in \Omega_{i}} p_{i}[z]^{1-\sigma}}
$$


The exact price index is defined as cost (expenditure) to obtain an output value of 1 :

$$
P_{i}\left[N_{i}^{\frac{\mu-1}{\sigma}} \int_{z \in \Omega_{i}} q_{i}[z]^{\frac{\sigma-1}{\sigma}}\right]^{\frac{\sigma}{\sigma-1}}=1
$$

Using the first-order condition and solving for $P_{i}$, we obtain

$$
\begin{aligned}
P_{i} N_{i}^{\frac{\mu-1}{\sigma-1}}\left[\int_{z \in \Omega_{i}} q_{i}[z]^{\frac{\sigma-1}{\sigma}}\right]^{\frac{\sigma}{\sigma-1}} & =1 \\
P_{i} N_{i}^{\frac{\mu-1}{\sigma-1}} \frac{\left[\int_{z \in \Omega_{i}} p_{i}[z]^{1-\sigma}\right]^{\frac{\sigma}{\sigma-1}}}{\int_{z \in \Omega_{i}} p_{i}[z]^{1-\sigma}} & =1 \\
P_{i} & =N_{i}^{-\frac{\mu-1}{\sigma-1}}\left[\int_{z \in \Omega_{i}} p_{i}[z]^{1-\sigma}\right]^{-\frac{1}{\sigma-1}} .
\end{aligned}
$$

Then, profit-maximizing demand for input $z$ is given by

$$
q_{i}[z]=E_{i} P_{i}^{\sigma-1} N_{i}^{\mu-1} p_{i}[z]^{-\sigma} .
$$

Intermediate input producers. An intermediate input producer with productivity $\varphi$ in country $i$ sets the profit-maximizing price $p_{i j}[\varphi]$ for each market $j$, taking as given demand of the final good producer in country $j$ for an input from $i$ and wages $w_{i}$ :

$$
\max _{q_{i j}[z]} \pi_{i j}[\varphi]=p_{i j}[z] q_{i j}[z]-w_{i} \frac{\tau_{i j} q_{i j}[z]}{\varphi}-w_{i} f_{i j}
$$

where $\tau_{i j} \geq 1$ denotes iceberg trade costs with $\tau_{i i}=1$, and $f_{i j}$ is the fixed cost of market access in terms of labor.

The first-order condition of the profit-maximization problem reads

$$
p_{i j}[z]+q_{i j}[z] \frac{\partial p_{i j}[z]}{\partial q_{i j}[z]}=\frac{\tau_{i j} w_{i}}{\varphi} .
$$

Noting that the elasticity of demand in prices is given by $\sigma$, we obtain the profit maximizing price

$$
p_{i j}[z]=\frac{\tau_{i j} w_{i}}{\rho \varphi}
$$


which is the standard markup $1 / \rho \equiv \sigma /(\sigma-1)$ over marginal cost $\tau_{i j} w_{i} / \varphi$.

Substituting the profit-maximizing price back into the expression for profits, we obtain

$$
\begin{aligned}
\pi_{i j}[\varphi] & =p_{i j}[\varphi] q_{i j}[\varphi]-w_{i} \frac{\tau_{i j} q_{i j}[\varphi]}{\varphi}-w_{i} f_{i j} \\
& =p_{i j}[\varphi] q_{i j}[\varphi]-\frac{(\sigma-1) p_{i j}[\varphi] q_{i j}[\varphi]}{\sigma}-w_{i} f_{i j} \\
& =r_{i j}[\varphi]\left(1-\frac{\sigma-1}{\sigma}\right)-w_{i} f_{i j} \\
& =\frac{r_{i j}[\varphi]}{\sigma}-w_{i} f_{i j}
\end{aligned}
$$

where $r_{i j}[\varphi] \equiv p_{i j}[\varphi] q_{i j}[\varphi]$ denote revenue of firm $\varphi$ located in $i$ from selling to the final good producer in $j$.

Let $\varphi_{i j}^{*}$ denote the cutoff firm in country $i$ that makes zero profits from selling to $j$. The threshold $\varphi_{i j}^{*}$ is determined by

$$
\pi_{i j}\left[\varphi_{i j}^{*}\right]=0 \Longleftrightarrow r_{i j}\left[\varphi_{i j}^{*}\right]=\sigma w_{i} f_{i j}
$$

Using optimal demand to substitute out the quantity and the pricing rule to substitute the price from the revenue function, we obtain the zero cutoff profit condition

$$
E_{j} N_{j}^{\mu-1}\left(\frac{\rho P_{j}}{\tau_{i j} w_{i}} \varphi_{i j}^{*}\right)^{\sigma-1}=\sigma w_{i} f_{i j}
$$

Comparing cutoffs for domestic and foreign firms in country $j$, we obtain

$$
\begin{aligned}
\frac{E_{j} N_{j}^{\mu-1}\left(\frac{\rho P_{j}}{\tau_{i j} w_{i}} \varphi_{i j}^{*}\right)^{\sigma-1}}{E_{j} N_{j}^{\mu-1}\left(\frac{\rho P_{j}}{w_{j}} \varphi_{j j}^{*}\right)^{\sigma-1}} & =\frac{\sigma w_{i} f_{i j}}{\sigma w_{j} f_{j j}} \\
\left(\frac{\varphi_{i j}^{*}}{\varphi_{j j}^{*}}\right)^{\sigma-1} & =\tau_{i j}^{\sigma-1} \frac{f_{i j}}{f_{j j}}\left(\frac{w_{i}}{w_{j}}\right)^{\sigma},
\end{aligned}
$$

where we have used $f^{x}=f_{i j}$ and $f^{d}=f_{j j}$. The relative attractiveness of country $j$ is driven by variable trade costs, the fixed cost differential, and the cost differential. 
Price index. Recall that the price index $P_{i}$ satisfies:

$$
P_{i}^{1-\sigma}=N_{i}^{\mu-1}\left(M_{i}^{e} \int_{\varphi_{i i}^{*}} p_{i i}[\varphi]^{1-\sigma} d G[\varphi]+M_{j}^{e} \int_{\varphi_{j i}^{*}} p_{j i}[\varphi]^{1-\sigma} d G[\varphi]\right),
$$

where $M_{i}^{e}$ denotes the mass of successful entrants in country $i$. Using the pricing rule and the Pareto distribution $G[\varphi]=1-\varphi^{-\beta}$, we find

$$
\begin{aligned}
P_{i}^{1-\sigma} & =N_{i}^{\mu-1}\left(M_{i}^{e}\left(\frac{w_{i}}{\rho}\right)^{1-\sigma} \int_{\varphi_{i i}^{*}} \varphi^{\sigma-1} d G[\varphi]+M_{j}^{e}\left(\frac{\tau_{j i} w_{j}}{\rho}\right)^{1-\sigma} \int_{\varphi_{j i}^{*}} \varphi^{\sigma-1} d G[\varphi]\right) \\
& =N_{i}^{\mu-1}\left(M_{i}^{e}\left(\frac{w_{i}}{\rho}\right)^{1-\sigma} \beta \int_{\varphi_{i i}^{*}} \varphi^{\sigma-1} \varphi^{-\beta-1} d \varphi+M_{j}^{e}\left(\frac{\tau_{j i} w_{j}}{\rho}\right)^{1-\sigma} \beta \int_{\varphi_{j i}^{*}} \varphi^{\sigma-1} \varphi^{-\beta-1} d \varphi\right) \\
& =N_{i}^{\mu-1}\left(M_{i}^{e}\left(\frac{w_{i}}{\rho}\right)^{1-\sigma} \frac{\beta}{\beta-(\sigma-1)}\left(\varphi_{i i}^{*}\right)^{\sigma-1-\beta}+M_{j}^{e}\left(\frac{\tau_{j i} w_{j}}{\rho}\right)^{1-\sigma} \frac{\beta}{\beta-(\sigma-1)}\left(\varphi_{j i}^{*}\right)^{\sigma-1-\beta}\right) \\
& =\frac{\beta}{\beta-(\sigma-1)} N_{i}^{\mu-1}\left(M_{i}^{e}\left(\frac{w_{i}}{\rho}\right)^{1-\sigma}\left(\varphi_{i i}^{*}\right)^{\sigma-1-\beta}+M_{j}^{e}\left(\frac{\tau_{j i} w_{j}}{\rho}\right)^{1-\sigma}\left(\varphi_{j i}^{*}\right)^{\sigma-1-\beta}\right) \\
& =\theta N_{i}^{\mu-1}\left(M_{i}^{e}\left(\varphi_{i i}^{*}\right)^{-\beta}\left(\frac{\rho \varphi_{i i}^{*}}{w_{i}}\right)^{\sigma-1}+M_{j}^{e}\left(\varphi_{j i}^{*}\right)^{-\beta}\left(\frac{\rho \varphi_{j i}^{*}}{\tau_{j i} w_{j}}\right)^{\sigma-1}\right) \\
& =\theta N_{i}^{\mu-1}\left(M_{i i}\left(\frac{\rho \varphi_{i i}^{*}}{w_{i}}\right)^{\sigma-1}+M_{j i}\left(\frac{\rho \varphi_{j i}^{*}}{\tau_{j i} w_{j}}\right)^{\sigma-1}\right)
\end{aligned}
$$

where we define $\theta \equiv \frac{\beta}{\beta-(\sigma-1)}>0$ and $M_{j i} \equiv \varphi_{j i}^{*} M_{j}^{e}$ denotes the mass of firms from $j$ active in $i$.

Free entry. Free entry implies that the probability of successful entry times expected profits earned from successful entry must be equal to the cost of entry

$$
\left(1-G\left[\varphi_{i i}^{*}\right]\right) \bar{\pi}_{i}=w_{i} f^{e}
$$

Expected profits of input suppliers located in $i$ (across purely domestic firms and exporters) are given by

$$
\bar{\pi}_{i}=\int_{\varphi_{i i}^{*}} \pi_{i i}[\varphi] \frac{d G[\varphi]}{1-G\left[\varphi_{i i}^{*}\right]}+\int_{\varphi_{i j}^{*}} \pi_{i j}[\varphi] \frac{d G[\varphi]}{1-G\left[\varphi_{i i}^{*}\right]}
$$


where we divide the density function by $\left(1-G\left[\varphi_{i i}^{*}\right]\right)$ in order to condition on the probability of successful entry. Using the expression for profits and noting that $r_{i j}[\varphi]=r_{i j}\left[\varphi_{i j}^{*}\right]\left(\frac{\varphi}{\varphi_{i j}^{*}}\right)^{\sigma-1}$, we obtain

$$
\begin{aligned}
\bar{\pi}_{i} & =\int_{\varphi_{i i}^{*}}\left(\frac{r_{i i}[\varphi]}{\sigma}-w_{i} f_{i i}\right) \frac{d G[\varphi]}{1-G\left[\varphi_{i i}^{*}\right]}+\int_{\varphi_{i j}^{*}}\left(\frac{r_{i j}[\varphi]}{\sigma}-w_{i} f_{i j}\right) \frac{d G[\varphi]}{1-G\left[\varphi_{i i}^{*}\right]} \\
& =\int_{\varphi_{i i}^{*}}\left(\frac{r_{i i}\left[\varphi_{i i}^{*}\right]}{\sigma}\left(\frac{\varphi}{\varphi_{i i}^{*}}\right)^{\sigma-1}-w_{i} f_{i i}\right) \frac{d G[\varphi]}{1-G\left[\varphi_{i i}^{*}\right]}+\int_{\varphi_{i j}^{*}}\left(\frac{r_{i j}\left[\varphi_{i j}^{*}\right]}{\sigma}\left(\frac{\varphi}{\varphi_{i j}^{*}}\right)^{\sigma-1}-w_{i} f_{i j}\right) \frac{d G[\varphi]}{1-G\left[\varphi_{i i}^{*}\right]} \\
& =\int_{\varphi_{i i}^{*}}\left(\frac{\sigma w_{i} f_{i i}}{\sigma}\left(\frac{\varphi}{\varphi_{i i}^{*}}\right)^{\sigma-1}-w_{i} f_{i i}\right) \frac{d G[\varphi]}{1-G\left[\varphi_{i i}^{*}\right]}+\int_{\varphi_{i j}^{*}}\left(\frac{\sigma w_{i} f_{i j}}{\sigma}\left(\frac{\varphi}{\varphi_{i j}^{*}}\right)^{\sigma-1}-w_{i} f_{i j}\right) \frac{d G[\varphi]}{1-G\left[\varphi_{i i}^{*}\right]} \\
& =w_{i} f_{i i} \int_{\varphi_{i i}^{*}}\left(\left(\frac{\varphi}{\varphi_{i i}^{*}}\right)^{\sigma-1}-1\right) \frac{d G[\varphi]}{1-G\left[\varphi_{i i}^{*}\right]}+w_{i} f_{i j} \int_{\varphi_{i j}^{*}}\left(\left(\frac{\varphi}{\varphi_{i j}^{*}}\right)^{\sigma-1}-1\right) \frac{d G[\varphi]}{1-G\left[\varphi_{i i}^{*}\right]}
\end{aligned}
$$

Employing the Pareto distribution, we have

$$
\begin{aligned}
\int_{\varphi_{i j}^{*}}\left(\left(\frac{\varphi}{\varphi_{i j}^{*}}\right)^{\sigma-1}-1\right) \frac{d G[\varphi]}{1-G\left[\varphi_{i i}^{*}\right]} & =\left(\varphi_{i i}^{*}\right)^{\beta} \beta \int_{\varphi_{i j}^{*}}\left(\left(\frac{\varphi}{\varphi_{i j}^{*}}\right)^{\sigma-1}-1\right) \varphi^{-\beta-1} d \varphi \\
& =\left(\varphi_{i i}^{*}\right)^{\beta} \beta\left(\left(\varphi_{i j}^{*}\right)^{1-\sigma} \int_{\varphi_{i j}^{*}} \varphi^{\sigma-1-\beta-1}-\int_{\varphi_{i j}^{*}} \varphi^{-\beta-1} d \varphi\right) \\
& =\left(\varphi_{i i}^{*}\right)^{\beta} \beta\left(\left(\varphi_{i j}^{*}\right)^{1-\sigma} \frac{\left(\varphi_{i j}^{*}\right)^{\sigma-1-\beta}}{\beta-(\sigma-1)}-\frac{1}{\beta}\left(\varphi_{i j}^{*}\right)^{-\beta}\right) \\
& =\left(\frac{\varphi_{i i}^{*}}{\varphi_{i j}^{*}}\right)^{\beta}\left(\frac{\beta}{\beta-(\sigma-1)}-1\right) \\
& =\left(\frac{\varphi_{i i}^{*}}{\varphi_{i j}^{*}}\right)^{\beta} \frac{\sigma-1}{\beta-(\sigma-1)}
\end{aligned}
$$

Substituting this expression back into the expression for expected profits, we obtain

$$
\begin{aligned}
\bar{\pi}_{i} & =w_{i} f_{i i}\left(\frac{\varphi_{i i}^{*}}{\varphi_{i i}^{*}}\right)^{\beta} \frac{\sigma-1}{\beta-(\sigma-1)}+w_{i} f_{i j}\left(\frac{\varphi_{i i}^{*}}{\varphi_{i j}^{*}}\right)^{\beta} \frac{\sigma-1}{\beta-(\sigma-1)} \\
& =(\theta-1) w_{i}\left(f_{i i}+\left(\frac{\varphi_{i i}^{*}}{\varphi_{i j}^{*}}\right)^{\beta} f_{i j}\right) \\
& =(\theta-1) w_{i}\left(f_{i i}+m_{i j} f_{i j}\right),
\end{aligned}
$$


where we define the export participation rate $m_{i j} \equiv \frac{1-G\left[\varphi_{i j}^{*}\right]}{1-G\left[\varphi_{i i}^{*}\right]}=\left(\frac{\varphi_{i i}^{*}}{\varphi_{i j}^{*}}\right)^{\beta}$.

Then, the free entry condition can be rewritten as

$$
\begin{aligned}
(\theta-1)\left(\varphi_{i i}^{*}\right)^{-\beta} w_{i}\left(f_{i i}+m_{i j} f_{i j}\right) & =w_{i} f^{e} \\
(\theta-1)\left(\varphi_{i i}^{*}\right)^{-\beta}\left(f_{i i}+m_{i j} f_{i j}\right) & =f^{e} .
\end{aligned}
$$

Note that the wage rate disappears as expected profits from domestic activity and exporting as well as entry fixed costs are proportional to the domestic wage.

Labor market clearing. Labor market clearing requires

$$
L_{i}=M_{i}^{e} f^{e}+M_{i}^{e}\left(\varphi_{i i}^{*}\right)^{-\beta} f_{i i}+M_{i}^{e}\left(\varphi_{i j}^{*}\right)^{-\beta} f_{i j}+M_{i}^{e} \int_{\varphi_{i i}^{*}} \frac{q_{i i}[\varphi]}{\varphi} d G[\varphi]+M_{i}^{e} \int_{\varphi_{i j}^{*}} \frac{\tau_{i j} q_{i j}[\varphi]}{\varphi} d G[\varphi]
$$

Using optimal demand, the pricing rule, and Pareto, we obtain

$$
\begin{aligned}
\int_{\varphi_{i j}^{*}} \frac{\tau_{i j} q_{i j}[\varphi]}{\varphi} d G[\varphi] & =E_{j} P_{j}^{\sigma-1} N_{j}^{\mu-1} \tau_{i j} \int_{\varphi_{i j}^{*}} \frac{p_{i j}[\varphi]^{-\sigma}}{\varphi} d G[\varphi] \\
& =E_{j} P_{j}^{\sigma-1} N_{j}^{\mu-1}\left(\frac{w_{i}}{\rho}\right)^{-\sigma} \tau_{i j}^{1-\sigma} \int_{\varphi_{i j}^{*}} \varphi^{\sigma-1} d G[\varphi] \\
& =E_{j} P_{j}^{\sigma-1} N_{j}^{\mu-1}\left(\frac{w_{i}}{\rho}\right)^{-\sigma} \tau_{i j}^{1-\sigma} \beta \int_{\varphi_{i j}^{*}} \varphi^{\sigma-1-\beta-1} d \varphi \\
& =E_{j} P_{j}^{\sigma-1} N_{j}^{\mu-1}\left(\frac{w_{i}}{\rho}\right)^{-\sigma} \tau_{i j}^{1-\sigma} \frac{\beta}{\beta-(\sigma-1)}\left(\varphi_{i j}^{*}\right)^{\sigma-1-\beta} .
\end{aligned}
$$

Employing the zero cutoff profit condition to substitute out $\left(\varphi_{i j}^{*}\right)^{\sigma-1}$, we find

$$
\begin{aligned}
\int_{\varphi_{i j}^{*}} \frac{\tau_{i j} q_{i j}[\varphi]}{\varphi} d G[\varphi] & =E_{j} P_{j}^{\sigma-1} N_{j}^{\mu-1}\left(\frac{w_{i}}{\rho}\right)^{-\sigma} \tau_{i j}^{1-\sigma} \frac{\beta}{\beta-(\sigma-1)} \frac{\sigma w_{i}^{\sigma} f_{i j} \tau_{i j}^{\sigma-1} \rho^{1-\sigma}}{E_{j} N_{j}^{\mu-1}} P_{j}^{1-\sigma}\left(\varphi_{i j}^{*}\right)^{-\beta} \\
& =\frac{\beta \sigma \rho}{\beta-(\sigma-1)}\left(\varphi_{i j}^{*}\right)^{-\beta} f_{i j} \\
& =\frac{\beta(\sigma-1)}{\beta-(\sigma-1)}\left(\varphi_{i j}^{*}\right)^{-\beta} f_{i j}
\end{aligned}
$$


Using this expression in the labor market clearing condition, we have

$$
\begin{aligned}
L_{i} & =M_{i}^{e}\left[f^{e}+\left(\varphi_{i i}^{*}\right)^{-\beta} f_{i i}+\left(\varphi_{i j}^{*}\right)^{-\beta} f_{i j}+\frac{\beta(\sigma-1)}{\beta-(\sigma-1)}\left(\left(\varphi_{i i}^{*}\right)^{-\beta} f_{i i}+\left(\varphi_{i j}^{*}\right)^{-\beta} f_{i j}\right)\right] \\
& =M_{i}^{e}\left[f^{e}+\left(\frac{\beta(\sigma-1)}{\beta-(\sigma-1)}+1\right)\left(\left(\varphi_{i i}^{*}\right)^{-\beta} f_{i i}+\left(\varphi_{i j}^{*}\right)^{-\beta} f_{i j}\right)\right] .
\end{aligned}
$$

Using free entry, we obtain

$$
\begin{aligned}
L_{i} & =M_{i}^{e}\left[f^{e}+\left(\frac{\beta(\sigma-1)}{\beta-(\sigma-1)}+1\right)\left(\left(\varphi_{i i}^{*}\right)^{-\beta} f_{i i}+\left(\varphi_{i j}^{*}\right)^{-\beta} f_{i j}\right)\right] \\
& =M_{i}^{e}\left[f^{e}+\left(\frac{\beta(\sigma-1)}{\beta-(\sigma-1)}+1\right) \frac{f^{e}}{\theta-1}\right] \\
& =M_{i}^{e} f^{e}\left[1+\left(\frac{\beta(\sigma-1)}{\beta-(\sigma-1)}+1\right) \frac{\beta-(\sigma-1)}{\sigma-1}\right] \\
& =M_{i}^{e} f^{e}\left[1+\beta+\frac{\beta-(\sigma-1)}{\sigma-1}\right] \\
& =M_{i}^{e} f^{e} \frac{\sigma-1+\beta(\sigma-1)+\beta-(\sigma-1)}{\sigma-1} \\
& =M_{i}^{e} f^{e} \frac{\beta \sigma}{\sigma-1} \\
& =M_{i}^{e} f^{e} \frac{\beta}{\rho} .
\end{aligned}
$$

Hence, a constant share $\rho / \beta$ of workers is devoted to entry activity.

The mass of active firms $M_{i}$ is given by

$$
M_{i}=\left(1-G\left[\varphi_{i i}^{*}\right]\right) M_{i}^{e}
$$

Hence, labor market clearing implies

$$
M_{i}=\left(1-G\left[\varphi_{i i}^{*}\right]\right) \frac{\rho}{\beta} \frac{L_{i}}{f^{e}} .
$$

Balanced trade. Balanced trade requires that Home's value of imports (i.e., Foreign's value of exports) is equal to Home's value of exports:

$$
M_{j}^{e} \int_{\varphi_{j i}^{*}} r_{j i}[\varphi] d G[\varphi]=M_{i}^{e} \int_{\varphi_{i j}^{*}} r_{i j}[\varphi] d G[\varphi]
$$


Recall that $r_{i j}[\varphi]=r_{i j}\left[\varphi_{i j}^{*}\right]\left(\frac{\varphi}{\varphi_{i j}^{*}}\right)^{\sigma-1}$. Using this result, the zero cutoff profit condition, and assuming symmetric fixed costs $f_{i j}=f_{j i}$, we obtain

$$
\begin{aligned}
M_{j}^{e} \frac{r_{j i}\left[\varphi_{j i}^{*}\right]}{\left(\varphi_{j i}^{*}\right)^{\sigma-1}} \int_{\varphi_{j i}^{*}} \varphi^{\sigma-1} d G[\varphi] & =M_{i}^{e} \frac{r_{i j}\left[\varphi_{i j}^{*}\right]}{\left(\varphi_{i j}^{*}\right)^{\sigma-1}} \int_{\varphi_{i j}^{*}} \varphi^{\sigma-1} d G[\varphi] \\
M_{j}^{e} \frac{w_{j} f_{j i}}{\left(\varphi_{j i}^{*}\right)^{\sigma-1} \int_{\varphi_{j i}^{*}} \varphi^{\sigma-1-\beta-1} d \varphi} & =M_{i}^{e} \frac{w_{i} f_{i j}}{\left(\varphi_{i j}^{*}\right)^{\sigma-1} \int_{\varphi_{i j}^{*}} \varphi^{\sigma-1-\beta-1} d \varphi} \\
M_{j}^{e} \frac{w_{j}}{\left(\varphi_{j i}^{*}\right)^{\sigma-1}}\left(\varphi_{j i}^{*}\right)^{\sigma-1-\beta} & =M_{i}^{e} \frac{w_{i}}{\left(\varphi_{i j}^{*}\right)^{\sigma-1}}\left(\varphi_{i j}^{*}\right)^{\sigma-1-\beta} \\
M_{j}^{e} w_{j}\left(\varphi_{j i}^{*}\right)^{-\beta} & =M_{i}^{e} w_{i}\left(\varphi_{i j}^{*}\right)^{-\beta} .
\end{aligned}
$$

Recognizing that $M_{i}^{e}$ is proportional to $L_{i}$ and using $\omega \equiv w_{H} / w_{F}$ and $L \equiv L_{H} / L_{F}$, balanced trade requires

$$
\omega=L^{-1}\left(\frac{\varphi_{F H}^{*}}{\varphi_{H F}^{*}}\right)^{-\beta}
$$

Mass of available inputs. By definition, the mass of available inputs is given by

$$
\begin{aligned}
N_{i} & =\left(\varphi_{i i}^{*}\right)^{-\beta} M_{i}^{e}+\left(\varphi_{j i}^{*}\right)^{-\beta} M_{j}^{e} \\
& =\left(\varphi_{i i}^{*}\right)^{-\beta} M_{i}^{e}\left[1+\left(\frac{\varphi_{j i}^{*}}{\varphi_{i i}^{*}}\right)^{-\beta} \frac{M_{j}^{e}}{M_{i}^{e}}\right]
\end{aligned}
$$

Recalling that the mass of entrants $M_{i}^{e}$ is proportional to country size, we can write the mass of available inputs in relative form as

$$
\frac{N_{i}}{N_{j}}=\frac{L_{i}}{L_{j}}\left(\frac{\varphi_{i i}^{*}}{\varphi_{j j}^{*}}\right)^{-\beta} \frac{1+\left(\frac{\varphi_{j i}^{*}}{\varphi_{i i}^{*}}\right)^{-\beta} \frac{L_{j}}{L_{i}}}{1+\left(\frac{\varphi_{i j}^{*}}{\varphi_{j j}^{*}}\right)^{-\beta} \frac{L_{i}}{L_{j}}}
$$

Using the zero cutoff profit conditions in relative form to substitute out $\varphi_{j i}^{*} / \varphi_{i i}^{*}$ and employing 
the definition $\eta=\tau^{-\beta}\left(\frac{f^{c}}{f^{d}}\right)^{1-\frac{\beta}{\sigma-1}}$, we obtain

$$
\begin{aligned}
N & \equiv \frac{N_{H}}{N_{F}}=L \times \chi \times \frac{1+\tau^{-\beta}\left(\frac{f^{x}}{f^{d}}\right)^{-\frac{\beta}{\sigma-1}} \omega^{\frac{\beta}{\rho}} L^{-1}}{1+\tau^{-\beta}\left(\frac{f^{x}}{f^{d}}\right)^{-\frac{\beta}{\sigma-1}} \omega^{-\frac{\beta}{\rho}} L} \\
& =L \times \chi \times \frac{\frac{f^{x}}{f^{d}}+\tau^{-\beta}\left(\frac{f^{x}}{f^{d}}\right)^{1-\frac{\beta}{\sigma-1}} \omega^{\frac{\beta}{\rho}} L^{-1}}{\frac{f^{x}}{f^{d}}+\tau^{-\beta}\left(\frac{f^{x}}{f^{d}}\right)^{1-\frac{\beta}{\sigma-1}} \omega^{-\frac{\beta}{\rho}} L} \\
& =L \times \chi \times \frac{\frac{f^{x}}{f^{d}}+\eta \omega^{\frac{\beta}{\rho}} L^{-1}}{\frac{f^{x}}{f^{d}}+\eta \omega^{-\frac{\beta}{\rho}} L} .
\end{aligned}
$$

Relative average productivity. By definition, we have

$$
\tilde{\varphi}_{i}^{\sigma-1}=\frac{1}{N_{i}}\left[M_{i i} \tilde{\varphi}_{i i}^{\sigma-1}+M_{j i}\left(\frac{\tilde{\varphi}_{j i}}{\tau_{j i} w_{j} / w_{i}}\right)^{\sigma-1}\right]
$$

Using $M_{j i}=\left(\varphi_{j i}^{*}\right)^{-\beta} M_{i}^{e}$, we obtain

$$
\tilde{\varphi}_{i}^{\sigma-1}=\frac{1}{N_{i}}\left[M_{i}^{e}\left(\varphi_{i i}^{*}\right)^{-\beta} \tilde{\varphi}_{i i}^{\sigma-1}+M_{j}^{e}\left(\varphi_{j i}^{*}\right)^{-\beta}\left(\frac{\tilde{\varphi}_{j i}}{\tau_{j i} w_{j} / w_{i}}\right)^{\sigma-1}\right] .
$$

In relative from, average productivity $\tilde{\varphi}_{i} / \tilde{\varphi}_{j}$ satisfies

$$
\left(\frac{\tilde{\varphi}_{i}}{\tilde{\varphi}_{j}}\right)^{\sigma-1}=\frac{N_{j}}{N_{i}} \frac{M_{i}^{e}}{M_{j}^{e}} \frac{\left(\varphi_{i i}^{*}\right)^{-\beta} \tilde{\varphi}_{i i}^{\sigma-1}}{\left(\varphi_{j j}^{*}\right)^{-\beta} \tilde{\varphi}_{j j}^{\sigma-1}} \frac{1+\frac{M_{j}^{e}}{M_{i}^{e}}\left(\frac{\varphi_{j i}^{*}}{\varphi_{i i}^{*}}\right)^{-\beta}\left(\frac{\tilde{\varphi}_{j i} / \tilde{\varphi}_{i i}}{\tau_{j i} w_{j} / w_{i}}\right)^{\sigma-1}}{M_{j}^{e}\left(\frac{\varphi_{i j}^{*}}{\varphi_{j j}^{*}}\right)^{-\beta}\left(\frac{\tilde{\varphi}_{i j} / \tilde{\varphi}_{j j}}{\tau_{i j} w_{i} / w_{j}}\right)^{\sigma-1}} .
$$

Recalling that the mass of entrants $M_{i}^{e}$ is proportional to country size $L_{i}$ and that under Pareto, average productivity $\tilde{\varphi}_{j i}$ is proportional to entry cutoff $\varphi_{j i}^{*}$, we obtain

$$
\left(\frac{\tilde{\varphi}_{i}}{\tilde{\varphi}_{j}}\right)^{\sigma-1}=\frac{N_{j}}{N_{i}} \frac{L_{i}}{L_{j}} \frac{\left(\varphi_{i i}^{*}\right)^{\sigma-1-\beta}}{\left(\varphi_{j j}^{*}\right)^{\sigma-1-\beta}} \frac{1+\frac{L_{j}}{L_{i}}\left(\frac{\varphi_{j i}^{*}}{\varphi_{i i}^{*}}\right)^{\sigma-1-\beta}\left(\frac{w_{i}}{\tau_{j i} w_{j}}\right)^{\sigma-1}}{1+\frac{L_{i}}{L_{j}}\left(\frac{\varphi_{i j}^{*}}{\varphi_{j j}^{*}}\right)^{\sigma-1-\beta}\left(\frac{w_{j}}{\tau_{i j} w_{i}}\right)^{\sigma-1}} .
$$

Using the relative zero cutoff profit conditions to substitute out $\varphi_{j i}^{*} / \varphi_{i i}^{*}$ and employing the 
definition $\eta=\tau^{-\beta}\left(\frac{f^{c}}{f^{d}}\right)^{1-\frac{\beta}{\sigma-1}}$, we obtain

$$
\begin{aligned}
\left(\frac{\tilde{\varphi}_{i}}{\tilde{\varphi}_{j}}\right)^{\sigma-1} & =\frac{N_{j}}{N_{i}} \frac{L_{i}}{L_{j}}\left(\frac{\varphi_{i i}^{*}}{\varphi_{j j}^{*}}\right)^{\sigma-1-\beta} \frac{1+\frac{L_{j}}{L_{i}}\left(\tau\left(\frac{f^{x}}{f^{d}}\right)^{\frac{1}{\sigma-1}}\left(\frac{w_{i}}{w_{j}}\right)^{-\frac{1}{\rho}}\right)^{\sigma-1-\beta}\left(\frac{w_{i}}{\tau_{j i} w_{j}}\right)^{\sigma-1}}{1+\frac{L_{i}}{L_{j}}\left(\tau\left(\frac{f^{x}}{f^{d}}\right)^{\frac{1}{\sigma-1}}\left(\frac{w_{i}}{w_{j}}\right)^{\frac{1}{\rho}}\right)^{\sigma-1-\beta}\left(\frac{w_{j}}{\tau_{i j} w_{i}}\right)^{\sigma-1}} \\
& =\frac{N_{j}}{N_{i}} \frac{L_{i}}{L_{j}}\left(\frac{\varphi_{i i}^{*}}{\varphi_{j j}^{*}}\right)^{\sigma-1-\beta} \frac{1+\eta \frac{L_{j}}{L_{i}}\left(\frac{w_{i}}{w_{j}}\right)^{\frac{\beta-\rho}{\rho}}}{1+\eta \frac{L_{i}}{L_{j}}\left(\frac{w_{i}}{w_{j}}\right)^{-\frac{\beta-\rho}{\rho}}} .
\end{aligned}
$$

Hence, we have

$$
\tilde{\varphi}^{\sigma-1}=\frac{L}{N} \chi^{1-\frac{\sigma-1}{\beta}} \frac{1+\eta L^{-1} \omega^{\frac{\beta-\rho}{\rho}}}{1+\eta L \omega^{-\frac{\beta-\rho}{\rho}}} .
$$

Using the zero cutoff profit conditions in relative form and the trade balance condition, we will show below that $\chi=L \omega^{-\frac{2 \beta-\rho}{\rho}}$ ("ex post profitability curve"). Using this relationship to substitute out relative country size from the last term in the above expression, we obtain

$$
\tilde{\varphi}^{\sigma-1}=\frac{L}{N} \chi^{1-\frac{\sigma-1}{\beta}} \frac{1+\eta \chi^{-1} \omega^{-\frac{\beta}{\rho}}}{1+\eta \chi \omega^{\frac{\beta}{\rho}}} .
$$

Starting from the free entry conditions in relative form and using the zero profit conditions in relative form, we will also show that $\chi=\frac{1+\eta \chi \omega^{\frac{\beta}{\rho}}}{1+\eta \chi^{-1} \omega^{-\frac{\beta}{\rho}}}$ ("ex ante profitability curve"). Using this expression, we realize that the last term in the above expression is equal to $\chi^{-1}$. Collecting terms, we obtain

$$
\tilde{\varphi}=\left(\frac{L}{N}\right)^{\frac{1}{\sigma-1}} \chi^{-\frac{1}{\beta}}
$$

Total factor productivity. Total factor productivity is a measure of well-being in the economy. It is defined as

$$
\operatorname{TFP}_{i}=\frac{w_{i}}{P_{i}}
$$


where the price index $P_{i}$ satisfies

$$
\begin{aligned}
P_{i}^{1-\sigma} & =\rho^{\sigma-1} \theta N_{i}^{\mu-1}\left(M_{i i}\left(\frac{\varphi_{i i}^{*}}{w_{i}}\right)^{\sigma-1}+M_{j i}\left(\frac{\varphi_{j i}^{*}}{\tau_{j i} w_{j}}\right)^{\sigma-1}\right) \\
& =\rho^{\sigma-1} N_{i}^{\mu} w_{i}^{1-\sigma}\left(\frac{M_{i i}}{N_{i}} \theta\left(\varphi_{i i}^{*}\right)^{\sigma-1}+\frac{M_{j i}}{N_{i}} \theta\left(\frac{\varphi_{j i}^{*}}{\tau_{j i} w_{j} / w_{i}}\right)^{\sigma-1}\right) .
\end{aligned}
$$

Under Pareto, the average productivity of suppliers located in $j$ serving country $i$ satisfies

$$
\begin{aligned}
\tilde{\varphi}_{j i}^{\sigma-1} & \equiv \int_{\varphi_{j i}^{*}} \varphi^{\sigma-1} \frac{d G[\varphi]}{1-G\left[\varphi_{j i}^{*}\right]} \\
& =\beta\left(\varphi_{j i}^{*}\right)^{\beta} \int_{\varphi_{j i}^{*}} \varphi^{\sigma-1-\beta-1} d \varphi \\
& =\frac{\beta}{\beta-(\sigma-1)}\left(\varphi_{j i}^{*}\right)^{\sigma-1} .
\end{aligned}
$$

Using this definition to substitute out $\left(\varphi_{j i}^{*}\right)^{\sigma-1}$ from the expression for the price index and noting that $\theta \equiv \frac{\beta}{\beta-(\sigma-1)}$, we obtain

$$
P_{i}^{1-\sigma}=\rho^{\sigma-1} N_{i}^{\mu} w_{i}^{1-\sigma}\left(\frac{M_{i i}}{N_{i}} \tilde{\varphi}_{i i}^{\sigma-1}+\frac{M_{j i}}{N_{i}}\left(\frac{\tilde{\varphi}_{j i}}{\tau_{j i} w_{j} / w_{i}}\right)^{\sigma-1}\right) .
$$

We now define average productivity $\tilde{\varphi}_{i}$ satisfying

$$
\tilde{\varphi}_{i}^{\sigma-1} \equiv \frac{M_{i i}}{N_{i}} \tilde{\varphi}_{i i}^{\sigma-1}+\frac{M_{j i}}{N_{i}}\left(\frac{\tilde{\varphi}_{j i}}{\tau_{j i} w_{j} / w_{i}}\right)^{\sigma-1}
$$

Using this definition, we can rewrite TFP as

$$
\begin{aligned}
\operatorname{TFP}_{i} & =\frac{w_{i}}{\rho^{-1} N_{i}^{-\frac{\mu}{\sigma-1}} w_{i}\left(\frac{M_{i i}}{N_{i}} \tilde{\varphi}_{i i}^{\sigma-1}+\frac{M_{j i}}{N_{i}}\left(\frac{\tilde{\varphi}_{j i}}{\tau_{j i} w_{j} / w_{i}}\right)^{\sigma-1}\right)^{-\frac{1}{\sigma-1}}} \\
& =\rho N_{i}^{\frac{\mu}{\sigma-1}}\left(\frac{M_{i i}}{N_{i}} \tilde{\varphi}_{i i}^{\sigma-1}+\frac{M_{j i}}{N_{i}}\left(\frac{\tilde{\varphi}_{j i}}{\tau_{j i} w_{j} / w_{i}}\right)^{\sigma-1}\right)^{\frac{1}{\sigma-1}} \\
& =\rho N_{i}^{\frac{\mu}{\sigma-1}} \tilde{\varphi}_{i} .
\end{aligned}
$$


Ex post profitability curve. In order to derive the ex post profitability curve, we use the zero cutoff profit conditions in relative form and the trade balance condition.

Recall that the relative attractiveness of market $j$ for foreign firms is given by

$$
\left(\frac{\varphi_{i j}^{*}}{\varphi_{j j}^{*}}\right)^{\sigma-1}=\tau_{i j}^{\sigma-1} \frac{f_{i j}}{f_{j j}}\left(\frac{w_{i}}{w_{j}}\right)^{\sigma}
$$

Using Home as the target country, $f_{H H}=f^{d}$ and $f_{F H}=f^{x}$, and $\omega \equiv w_{H} / w_{F}$, we have

$$
\left(\frac{\varphi_{F H}^{*}}{\varphi_{H H}^{*}}\right)^{\sigma-1}=\tau_{F H}^{\sigma-1} \frac{f^{x}}{f^{d}} \omega^{-\sigma} \Leftrightarrow \varphi_{F H}^{*}=\tau_{F H}\left(\frac{f^{x}}{f^{d}}\right)^{\frac{1}{\sigma-1}} \omega^{-\frac{\sigma}{\sigma-1}} \varphi_{H H}^{*} .
$$

Similarly, for Foreign as the target country, we obtain

$$
\left(\frac{\varphi_{H F}^{*}}{\varphi_{F F}^{*}}\right)^{\sigma-1}=\tau_{H F}^{\sigma-1} \frac{f^{x}}{f^{d}} \omega^{\sigma} \Leftrightarrow \varphi_{H F}^{*}=\tau_{H F}\left(\frac{f^{x}}{f^{d}}\right)^{\frac{1}{\sigma-1}} \omega^{\frac{\sigma}{\sigma-1}} \varphi_{F F}^{*} .
$$

Using these expressions to substitute out foreign entry cutoffs from the trade balance condition and assuming symmetry in variable trade costs, we obtain

$$
\begin{aligned}
\omega & =L^{-1}\left(\frac{\varphi_{F H}^{*}}{\varphi_{H F}^{*}}\right)^{-\beta} \\
& =L^{-1}\left(\frac{\tau_{F H}\left(\frac{f^{x}}{f^{d}}\right)^{\frac{1}{\sigma-1}} \omega^{-\frac{\sigma}{\sigma-1}} \varphi_{H H}^{*}}{\tau_{H F}\left(\frac{f^{x}}{f^{d}}\right)^{\frac{1}{\sigma-1}} \omega^{\frac{\sigma}{\sigma-1}} \varphi_{F F}^{*}}\right)^{-\beta} \\
& =L^{-1} \omega^{\frac{2 \beta}{\rho}}\left(\frac{\varphi_{H H}^{*}}{\varphi_{F F}^{*}}\right)^{-\beta} .
\end{aligned}
$$

Defining the relative probability of successful entry (from Home's perspective) $\chi \equiv\left(\varphi_{F F}^{*} / \varphi_{H H}^{*}\right)^{\beta}$, we have

$$
\omega=L^{-1} \omega^{-\frac{2 \beta}{\rho}} \chi \Rightarrow \chi[\omega]=L \omega^{1-\frac{2 \beta}{\rho}}=L \omega^{-\frac{2 \beta-\rho}{\rho}}
$$

We have $\chi[1]=L$ and $\lim _{\omega \rightarrow \infty} \chi[\omega]=0$. The ex post profitability curve implies a downward- 
sloping and convex relationship between $\chi$ and $\omega$ as

$$
\begin{aligned}
\frac{\partial \chi[\omega]}{\partial \omega} & =-\frac{2 \beta-\rho}{\rho} L \omega^{-\frac{2 \beta}{\rho}}<0 \text { and } \\
\frac{\partial^{2} \chi[\omega]}{\partial \omega^{2}} & =\frac{2 \beta-\rho}{\rho} \frac{2 \beta}{\rho} L \omega^{-\frac{2 \beta}{\rho}-1}>0
\end{aligned}
$$

Ex ante profitability curve. In order to derive the ex ante profitability curve, we start from the free entry conditions in relative form. We then use the zero cutoff profit conditions substitute out export participation rates.

In relative terms, the free entry condition reads:

$$
\frac{(\theta-1)\left(\varphi_{i i}^{*}\right)^{-\beta}\left(f_{i i}+m_{i j} f_{i j}\right)}{(\theta-1)\left(\varphi_{j j}^{*}\right)^{-\beta}\left(f_{j j}+m_{j i} f_{j i}\right)}=\frac{f^{e}}{f^{e}} .
$$

Assuming symmetry in fixed costs $\left(f_{i i}=f_{j j}=f^{d}\right.$ and $\left.\mathrm{f}_{j i}=f_{i j}=f^{x}\right)$ and employing $\chi \equiv$ $\left(\varphi_{F F}^{*} / \varphi_{H H}^{*}\right)^{\beta}$, we have

$$
\chi=\frac{f^{d}+m_{F H} f^{x}}{f^{d}+m_{H F} f^{x}}=\frac{1+\left(\frac{\varphi_{F H}^{*}}{\varphi_{F F}^{*}}\right)^{-\beta} \frac{f^{x}}{f^{d}}}{1+\left(\frac{\varphi_{H F}^{*}}{\varphi_{H H}^{*}}\right)^{-\beta} \frac{f^{x}}{f^{d}}} .
$$

Using the zero cutoff profit conditions in relative form to substitute out export cutoffs, we obtain

$$
\begin{aligned}
\chi & =\frac{1+\left(\tau_{F H}\left(\frac{f^{x}}{f^{d}}\right)^{\frac{1}{\sigma-1}} \omega^{-\frac{\sigma}{\sigma-1} \frac{\varphi_{H H}^{*}}{\varphi_{F F}^{*}}}\right)^{-\beta} \frac{f^{x}}{f^{d}}}{1+\left(\tau_{H F}\left(\frac{f^{x}}{f^{d}}\right)^{\frac{1}{\sigma-1}} \omega^{\frac{\sigma}{\sigma-1}} \frac{\varphi_{F F}^{*}}{\varphi_{H H}^{*}}\right)^{-\beta} \frac{f^{x}}{f^{d}}} \\
& =\frac{1+\eta \omega^{\frac{\beta}{\rho}} \chi}{1+\eta \omega^{-\frac{\beta}{\rho}} \chi^{-1}},
\end{aligned}
$$


where we define the freeness of trade as $\eta \equiv \tau^{-\beta}\left(\frac{f^{x}}{f^{d}}\right)^{1-\frac{\beta}{\sigma-1}}$. We next solve for $\chi$ :

$$
\begin{aligned}
\chi\left(1+\eta \omega^{-\frac{\beta}{\rho}} \chi^{-1}\right) & =1+\eta \omega^{\frac{\beta}{\rho}} \chi \\
\chi+\eta \omega^{-\frac{\beta}{\rho}} & =1+\eta \omega^{\frac{\beta}{\rho}} \chi \\
\chi\left(1-\eta \omega^{\frac{\beta}{\rho}}\right) & =1-\eta \omega^{-\frac{\beta}{\rho}} \Rightarrow \\
\chi[\omega] & =\frac{1-\eta \omega^{-\frac{\beta}{\rho}}}{1-\eta \omega^{\frac{\beta}{\rho}}} .
\end{aligned}
$$

We have $\chi[1]=1$. Moreover, the ex ante profitability curve features an asymptote at $\omega=$ $\eta^{-\frac{\rho}{\beta}}$ such that $\omega<\eta^{-\frac{\rho}{\beta}}$.

Totally differentiating $\chi$, we obtain

$$
\frac{d \ln \chi}{d \ln \omega}=\frac{\beta}{\rho}\left(\frac{\eta \omega^{-\frac{\beta}{\rho}}}{1-\eta \omega^{-\frac{\beta}{\rho}}}+\frac{\eta \omega^{\frac{\beta}{\rho}}}{1-\eta \omega^{\frac{\beta}{\rho}}}\right)>0 .
$$

Hence, $\chi$ is strictly increasing in $\omega$ as long as $\eta>0$. With $\eta=0$, the ex ante profitability curve is a horizontal line at $\chi=1$.

In order to obtain the sign of the second derivative, we rewrite

$$
\frac{\partial \chi}{\partial \omega}=\frac{\beta \eta}{\rho} \frac{\chi}{\omega}\left(\frac{\omega^{-\frac{\beta}{\rho}}}{1-\eta \omega^{-\frac{\beta}{\rho}}}+\frac{\omega^{\frac{\beta}{\rho}}}{1-\eta \omega^{\frac{\beta}{\rho}}}\right) .
$$

Then,

$$
\begin{aligned}
\frac{d \ln \frac{\partial \chi}{\partial \omega}}{d \ln \omega} & =\frac{d \ln \chi}{d \ln \omega}-1-\frac{\beta}{\rho}+\frac{\beta}{\rho}-\frac{\beta}{\rho} \frac{\eta \omega^{-\frac{\beta}{\rho}}}{1-\eta \omega^{-\frac{\beta}{\rho}}}+\frac{\beta}{\rho} \frac{\eta \omega^{\frac{\beta}{\rho}}}{1-\eta \omega^{\frac{\beta}{\rho}}} \\
& =\frac{d \ln \chi}{d \ln \omega}-1+\frac{\beta}{\rho}\left(\frac{\eta \omega^{\frac{\beta}{\rho}}}{1-\eta \omega^{\frac{\beta}{\rho}}}-\frac{\eta \omega^{-\frac{\beta}{\rho}}}{1-\eta \omega^{-\frac{\beta}{\rho}}}\right) \\
& =\frac{\beta}{\rho}\left(\frac{\eta \omega^{-\frac{\beta}{\rho}}}{1-\eta \omega^{-\frac{\beta}{\rho}}}+\frac{\eta \omega^{\frac{\beta}{\rho}}}{1-\eta \omega^{\frac{\beta}{\rho}}}\right)-1+\frac{\beta}{\rho}\left(\frac{\eta \omega^{\frac{\beta}{\rho}}}{1-\eta \omega^{\frac{\beta}{\rho}}}-\frac{\eta \omega^{-\frac{\beta}{\rho}}}{1-\eta \omega^{-\frac{\beta}{\rho}}}\right) \\
& =\frac{2 \beta}{\rho} \frac{\eta \omega^{\frac{\beta}{\rho}}}{1-\eta \omega^{\frac{\beta}{\rho}}}-1
\end{aligned}
$$


Strict concavity of the ex ante profitability curve requires

$$
\begin{aligned}
\frac{d \ln \frac{\partial \chi}{\partial \omega}}{d \ln \omega} & >0 \Leftrightarrow \\
\frac{2 \beta}{\rho} \eta \omega^{\frac{\beta}{\rho}} & >1-\eta \omega^{\frac{\beta}{\rho}} \\
\frac{2 \beta+\rho}{\rho} \eta \omega^{\frac{\beta}{\rho}} & >1 .
\end{aligned}
$$

Note that a lower bound of $\omega$ is $\omega=1$. The ex ante profitability curve is concave in $\omega=1$, if and only if

$$
\eta>\frac{\rho}{2 \beta+\rho} .
$$

Notice that when ex ante profitability curve is concave in $\omega=1$, then it is also concave for all $1<\omega<\eta^{-\frac{\rho}{\beta}}$.

Existence and uniqueness of equilibrium. Consider the ex ante and ex post profitability curves on the relevant interval. The ex ante profitability curve goes through the point $(\omega=$ $1, \chi[1]=1)$. Is is strictly increasing and, if $\eta>\frac{\rho}{2 \beta+\rho}$, concave, and features an asymptote at $\omega=\eta^{-\frac{\rho}{\beta}}$. The ex post profitability curve goes through the point $(\omega=1, \chi[1]=L)$ and is decreasing and concave with the $\operatorname{limit}_{\omega \rightarrow \infty} \chi[\omega]=0<1$. This situation is depicted in Figure 2 . Hence, the curves intersect at least and at most once, which proves existence and uniqueness of equilibrium. Moreover, it proves that in equilibrium, $\omega>1$ and $\chi>1$, if $L>1$.

Home market effect. Following the literature, we say that the economy exhibits a Home Market Effect (HME), if the relative mass of active firms a country is larger than relative endowments.

Recall that labor market clearing requires that

$$
M_{i}=\left(\varphi_{i i}^{*}\right)^{-\beta} \frac{\rho}{\beta} \frac{L_{i}}{f^{e}}
$$


Hence, the relative mass of active firms is given by

$$
M \equiv \frac{M_{H}}{M_{F}}=\frac{\left(\varphi_{H H}^{*}\right)^{-\beta}}{\left(\varphi_{F F}^{*}\right)^{-\beta}} \frac{L_{H}}{L_{F}}=\chi L
$$

A HME is present, if $\chi>1$. We have argued above that in equilibrium, $\chi>1$, such that a HME exists.

Note that our definition of the HME implies that the country with the larger share in world population hosts an over-proportional share of active firms:

$$
\begin{aligned}
\frac{M_{H}}{M_{F}} & >\frac{L_{H}}{L_{F}} \Leftrightarrow \frac{M_{F}}{M_{H}}<\frac{L_{F}}{L_{H}} \Leftrightarrow \frac{M_{F}}{M_{H}}+1<\frac{L_{F}}{L_{H}}+1 \\
& \Leftrightarrow \frac{1}{\frac{M_{F}}{M_{H}}+1}>\frac{1}{\frac{L_{F}}{L_{H}}+1} \Leftrightarrow \frac{M_{H}}{M_{H}+M_{F}}>\frac{L_{H}}{L_{H}+L_{F}} .
\end{aligned}
$$

The firm share $\phi \equiv \frac{M_{H}}{M_{H}+M_{F}}$ and the population share $\lambda \equiv \frac{L_{H}}{L_{H}+L_{F}}$ are positive transformations of, respectively, $M$ and $L$. Hence, in order to characterize how $\phi[\lambda]$, it is sufficient to characterize $M[L]$.

Recall that

$$
M[L]=\chi[L] L
$$

In totally differentiated form, we have

$$
\frac{d \ln M}{d \ln L}=\frac{d \ln \chi}{d \ln L}+1
$$

We have seen above that $\chi$ is increasing in $L$. Hence, $M$ is increasing in $L$ as well.

In order to pin down the size of this elasticity, we have to understand how $\chi$ is affected by endowment shocks in general equilibrium.

The ex post profitability curve implies that

$$
\frac{d \ln \chi}{d \ln L}=1-\frac{2 \beta-\rho}{\rho} \frac{d \ln \omega}{d \ln L}
$$

Equalizing the ex ante and the ex post profitability curves, we see that there is no closed form 
solution for the equilibrium wage. We can nevertheless study its behavior with the help of the implicit function theorem.

In order to solve for the elasticity of the equilibrium wage in relative country size, we define

$$
T B[\omega, L] \equiv L-\omega^{\frac{\beta-\rho}{\rho}} \frac{\omega^{\frac{\beta}{\rho}}-\eta}{1-\eta \omega^{\frac{\beta}{\rho}}} .
$$

Then

$$
\frac{\partial T B}{\partial L}=1
$$

and

$$
\begin{aligned}
& \frac{\partial T B}{\partial \omega}=-\frac{\beta-\rho}{\rho} \omega^{\frac{\beta-\rho}{\rho}}-1 \frac{\omega^{\frac{\beta}{\rho}}-\eta}{1-\eta \omega^{\frac{\beta}{\rho}}}-\omega^{\frac{\beta-\rho}{\rho}} \frac{\frac{\beta}{\rho} \omega^{\frac{\beta}{\rho}-1}\left(1-\eta \omega^{\frac{\beta}{\rho}}\right)+\eta \frac{\beta}{\rho} \omega^{\frac{\beta}{\rho}-1}\left(\omega^{\frac{\beta}{\rho}}-\eta\right)}{\left(1-\eta \omega^{\frac{\beta}{\rho}}\right)^{2}} \\
& =-\left(\frac{\beta-\rho}{\rho} \frac{L}{\omega}+\frac{L}{\omega} \frac{1}{\rho} \frac{\beta \omega^{\frac{\beta}{\rho}}\left(1-\eta \omega^{\frac{\beta}{\rho}}\right)+\eta \beta \omega^{\frac{\beta}{\rho}}\left(\omega^{\frac{\beta}{\rho}}-\eta\right)}{\left(\omega^{\frac{\beta}{\rho}}-\eta\right)\left(1-\eta \omega^{\frac{\beta}{\rho}}\right)}\right) \\
& =-\frac{L}{\rho \omega}\left(\beta-\rho+\frac{\beta\left(1-\eta \omega^{\frac{\beta}{\rho}}\right)+\eta \beta\left(\omega^{\frac{\beta}{\rho}}-\eta\right)}{\left(\omega^{\frac{\beta}{\rho}}-\eta\right)\left(1-\eta \omega^{\frac{\beta}{\rho}}\right)} \omega^{\frac{\beta}{\rho}}\right) \\
& =-\frac{L}{\rho \omega}\left(\beta-\rho+\frac{\beta-\beta \eta \omega^{\frac{\beta}{\rho}}+\eta \beta \omega^{\frac{\beta}{\rho}}-\beta \eta^{2}}{\left(\omega^{\frac{\beta}{\rho}}-\eta\right)\left(1-\eta \omega^{\frac{\beta}{\rho}}\right)} \omega^{\frac{\beta}{\rho}}\right) \\
& =-\frac{L}{\rho \omega}\left(\beta-\rho+\beta \frac{1-\eta^{2}}{\left(\omega^{\frac{\beta}{\rho}}-\eta\right)\left(1-\eta \omega^{\frac{\beta}{\rho}}\right)} \omega^{\frac{\beta}{\rho}}\right) \\
& =-\frac{L}{\rho \omega}\left(\beta+\beta \frac{\left(1-\eta^{2}\right) \omega^{\frac{\beta}{\rho}}}{\left(\omega^{\frac{\beta}{\rho}}-\eta\right)\left(1-\eta \omega^{\frac{\beta}{\rho}}\right)}-\rho\right) \\
& =-\frac{L}{\rho \omega}\left(\beta \frac{\left(\omega^{\frac{\beta}{\rho}}-\eta\right)\left(1-\eta \omega^{\frac{\beta}{\rho}}\right)+\left(1-\eta^{2}\right) \omega^{\frac{\beta}{\rho}}}{\left(\omega^{\frac{\beta}{\rho}}-\eta\right)\left(1-\eta \omega^{\frac{\beta}{\rho}}\right)}-\rho\right) \\
& =-\frac{L}{\rho \omega}\left(\beta \frac{\omega^{\frac{\beta}{\rho}}-\eta \omega^{\frac{2 \beta}{\rho}}-\eta+\eta^{2} \omega^{\frac{\beta}{\rho}}+\omega^{\frac{\beta}{\rho}}-\eta^{2} \omega^{\frac{\beta}{\rho}}}{\left(\omega^{\frac{\beta}{\rho}}-\eta\right)\left(1-\eta \omega^{\frac{\beta}{\rho}}\right)}-\rho\right) \\
& =-\frac{L}{\rho \omega}\left(\beta \frac{2 \omega^{\frac{\beta}{\rho}}-\eta\left(\omega^{\frac{2 \beta}{\rho}}+1\right)}{\left(\omega^{\frac{\beta}{\rho}}-\eta\right)\left(1-\eta \omega^{\frac{\beta}{\rho}}\right)}-\rho\right)
\end{aligned}
$$


Employing the implicit function theorem, we obtain

$$
\begin{aligned}
\frac{\partial \omega}{\partial L} & =-\frac{\frac{\partial T B}{\partial L}}{\frac{\partial T B}{\partial \omega}} \\
& =\frac{\rho \omega}{L}\left(\beta \frac{2 \omega^{\frac{\beta}{\rho}}-\eta\left(\omega^{\frac{2 \beta}{\rho}}+1\right)}{\left(\omega^{\frac{\beta}{\rho}}-\eta\right)\left(1-\eta \omega^{\frac{\beta}{\rho}}\right)}-\rho\right)^{-1}
\end{aligned}
$$

Hence,

$$
\frac{d \ln \omega}{d \ln L}=\rho\left(\beta \frac{2 \omega^{\frac{\beta}{\rho}}-\eta\left(\omega^{\frac{2 \beta}{\rho}}+1\right)}{\left(\omega^{\frac{\beta}{\rho}}-\eta\right)\left(1-\eta \omega^{\frac{\beta}{\rho}}\right)}-\rho\right)^{-1}
$$

In the neighborhood of a symmetric equilibrium, we have

$$
\begin{aligned}
\frac{d \ln \omega}{d \ln L} & =\frac{\rho}{\beta\left(1-\frac{1-\eta^{2}}{(\eta-1)(\eta-1)}\right)-\rho} \\
& =\frac{\rho}{\beta\left(1-\frac{1+\eta}{\eta-1}\right)-\rho} \\
& =\frac{\rho}{\beta \frac{\eta-1-1-\eta}{\eta-1}-\rho} \\
& =\frac{\rho}{\beta \frac{-2}{\eta-1}-\rho} \\
& =\frac{\rho}{\beta \frac{2}{1-\eta}-\rho}
\end{aligned}
$$

Slope of $M$ in symmetric equilibrium:

$$
\begin{aligned}
\frac{d \ln M}{d \ln L} & =1-\frac{2 \beta-\rho}{\rho} \frac{d \ln \omega}{d \ln L} \\
& =2-\frac{2 \beta-\rho}{\rho} \frac{d \ln \omega}{d \ln L} \\
& =2-\frac{2 \beta-\rho}{\rho} \frac{\rho}{\beta \frac{2}{1-\eta}-\rho} \\
& =2-\frac{(2 \beta-\rho)(1-\eta)}{2 \beta-\rho(1-\eta)} \\
& =\frac{4 \beta-2 \rho(1-\eta)-(2 \beta-\rho)(1-\eta)}{2 \beta-\rho(1-\eta)} \\
& =\frac{2 \beta(\eta+1)-\rho(1-\eta)}{2 \beta-\rho(1-\eta)} \\
& =1+\frac{2 \beta \eta}{2 \beta-\rho(1-\eta)} .
\end{aligned}
$$


Now consider the firm share $\phi \equiv M_{H} /\left(M_{H}+M_{F}\right)=\left(1+M^{-1}\right)^{-1}$ as a function of the large country's endowment share $\lambda \equiv L_{H} /\left(L_{H}+L_{F}\right)=\left(1+L^{-1}\right)^{-1}$ as illustrated in Figure 3.

We have

$$
\phi=\frac{1}{1+M^{-1}}=\frac{1}{1+(\chi L)^{-1}}=\frac{\chi}{\chi+\frac{1-\lambda}{\lambda}}=\frac{\chi}{\lambda \chi+1-\lambda} \lambda .
$$

Let

$$
\gamma \equiv \frac{\chi}{\lambda \chi+1-\lambda}
$$

denote the factor of over-proportionality. We now show that on the interval $\lambda \in\left(0.5, \lambda^{*}\right)$, an increase in Home's endowment share raises its firm share more than proportionally, i.e.,

$$
\phi^{\prime}[\lambda]>1,
$$

and on the interval $\lambda \in\left(\lambda^{*}, \bar{\lambda}\right)$ less than proportionally, where $\bar{\lambda} \equiv\left(1+\bar{L}^{-1}\right)^{-1}$ is the transformation of the relative size cutoff $\bar{L}$ into an endowment share.

Taking the derivative of $\gamma$, we obtain

$$
\frac{\partial \gamma}{\partial \lambda}=\frac{\frac{\partial \chi}{\partial \lambda}[1+\lambda(\chi-1)]-\chi\left(\chi+\lambda \frac{\partial \chi}{\partial \lambda}-1\right)}{[1+\lambda(\chi-1)]^{2}} .
$$

Then,

$$
\begin{aligned}
\epsilon_{\gamma} & \equiv \frac{\lambda \frac{\partial \gamma}{\partial \lambda}}{\gamma}=\frac{\frac{\partial \chi}{\partial \lambda} \lambda[1+\lambda(\chi-1)]-\lambda \chi\left(\chi+\lambda \frac{\partial \chi}{\partial \lambda}-1\right)}{1+\lambda(\chi-1)} \frac{1}{\chi} \\
& =\epsilon_{\chi}(1-\phi)-\phi\left(\frac{\chi-1}{\chi}\right),
\end{aligned}
$$

where $\epsilon_{\chi} \equiv \frac{\partial \chi}{\partial \lambda} \frac{\lambda}{\chi}$. We have $\epsilon_{\gamma}>0$, if and only if

$$
\epsilon_{\chi}>\frac{\phi}{1-\phi}\left(\frac{\chi-1}{\chi}\right) .
$$


An alternative way to write this is

$$
\epsilon_{\chi}>\frac{\frac{\chi \lambda}{1+\lambda \chi-\lambda}}{\frac{1-\lambda}{1+\lambda \chi-\lambda}}\left(\frac{\chi-1}{\chi}\right)=\frac{\lambda}{1-\lambda}(\chi-1) \Leftrightarrow \frac{\epsilon_{\chi}}{\chi-1}>\frac{\lambda}{1-\lambda} .
$$

The conjecture is that we can identify a downward- and an upward-sloping curve such that the left hand side and the right hand side are equal for some unique $\lambda^{*} \in(1 / 2,1)$.

Using $\epsilon_{\chi}=\frac{\partial \chi}{\partial \lambda} \frac{\lambda}{\chi}=\frac{\partial \chi}{\partial \omega} \frac{\omega}{\chi} \frac{\partial \omega}{\partial \lambda} \frac{\lambda}{\omega}$, we can rewrite the inequality as

$$
\frac{\frac{\partial \chi}{\partial \omega} \frac{\omega}{\chi}}{\chi-1}>\frac{\lambda}{1-\lambda} \frac{1}{\frac{\partial \omega}{\partial \lambda} \frac{\lambda}{\omega}}
$$

We prove that the left hand side is downward-sloping in $\omega$ and therefore downward-sloping in $\lambda$, whereas the right hand side is increasing in $\lambda$.

Consider the $\frac{\partial \chi}{\partial \omega} \frac{\omega}{\chi} /(\chi-1)$ locus. Recall that

$$
\frac{\partial \chi}{\partial \omega}=\frac{\eta \beta}{\rho} \frac{\left(\omega^{-\frac{\beta}{\rho}}-\eta\right)+\left(\omega^{\frac{\beta}{\rho}}-\eta\right)}{\omega\left(1-\eta \omega^{\frac{\beta}{\rho}}\right)^{2}}>0 .
$$

The elasticity of $\chi$ in $\omega$ is then

$$
\frac{\partial \chi}{\partial \omega} \frac{\omega}{\chi}=\frac{\eta \beta}{\rho} \frac{\left(\omega^{-\frac{\beta}{\rho}}-\eta\right)+\left(\omega^{\frac{\beta}{\rho}}-\eta\right)}{\left(1-\eta \omega^{\frac{\beta}{\rho}}\right)\left(1-\eta \omega^{-\frac{\beta}{\rho}}\right)}
$$

where

$$
\frac{\partial \chi}{\partial \omega} \frac{\omega}{\chi}=\epsilon_{\chi} /\left(\frac{\partial \omega}{\partial \lambda} \frac{\lambda}{\omega}\right)
$$

Moreover,

$$
\chi-1=\eta \frac{\omega^{\frac{\beta}{\rho}}-\omega^{-\frac{\beta}{\rho}}}{1-\eta \omega^{\frac{\beta}{\rho}}}>0,
$$

and therefore

$$
\frac{\frac{\partial \chi}{\partial \omega} \frac{\omega}{\chi}}{\chi-1}=\frac{\beta}{\rho} \frac{\left(\omega^{-\frac{\beta}{\rho}}-\eta\right)+\left(\omega^{\frac{\beta}{\rho}}-\eta\right)}{\left(1-\eta \omega^{-\frac{\beta}{\rho}}\right)\left(\omega^{\frac{\beta}{\rho}}-\omega^{-\frac{\beta}{\rho}}\right)}
$$


We conjecture that $\partial\left[\frac{\partial \chi}{\partial \omega} \frac{\omega}{\chi} /(\chi-1)\right] / \partial \omega<0$. To check this, define

$$
\begin{aligned}
f[\omega] & \equiv \omega^{-\frac{\beta}{\rho}}+\omega^{\frac{\beta}{\rho}}-2 \eta>0 \\
g[\omega] & \equiv\left(1-\eta \omega^{-\frac{\beta}{\rho}}\right)\left(\omega^{\frac{\beta}{\rho}}-\omega^{-\frac{\beta}{\rho}}\right) \\
& =\omega^{\frac{\beta}{\rho}}-\eta-\omega^{-\frac{\beta}{\rho}}+\eta \omega^{-\frac{2 \beta}{\rho}}>0
\end{aligned}
$$

We have to show that

$$
f^{\prime}[\omega] g[\omega]-f[\omega] g^{\prime}[\omega]<0
$$

where

$$
\begin{aligned}
& f^{\prime}[\omega]=-\frac{\beta}{\rho} \omega^{-\frac{\beta}{\rho}-1}+\frac{\beta}{\rho} \omega^{\frac{\beta}{\rho}-1}=\frac{\beta}{\rho}\left(\frac{\omega^{\frac{\beta}{\rho}}-\omega^{-\frac{\beta}{\rho}}}{\omega}\right)>0 \\
& g^{\prime}[\omega]=\frac{\beta}{\rho} \omega^{\frac{\beta}{\rho}-1}+\frac{\beta}{\rho} \omega^{-\frac{\beta}{\rho}-1}-\frac{2 \beta}{\rho} \eta \omega^{-\frac{2 \beta}{\rho}-1}=\frac{\beta}{\rho}\left(\frac{\omega^{\frac{\beta}{\rho}}+\omega^{-\frac{\beta}{\rho}}-2 \eta \omega^{-\frac{2 \beta}{\rho}}}{\omega}\right)>0 .
\end{aligned}
$$

The last inequality follows from $\omega<\eta^{-\frac{\rho}{\beta}}$.

We can rewrite the necessary condition as

$$
\frac{\beta}{\rho} \frac{1}{\omega}\left(\omega^{\frac{\beta}{\rho}}-\omega^{-\frac{\beta}{\rho}}\right)^{2}\left(1-\eta \omega^{-\frac{\beta}{\rho}}\right)<\frac{\beta}{\rho} \frac{1}{\omega}\left(\omega^{-\frac{\beta}{\rho}}+\omega^{\frac{\beta}{\rho}}-2 \eta\right)\left(\omega^{\frac{\beta}{\rho}}+\omega^{-\frac{\beta}{\rho}}-2 \eta \omega^{-\frac{2 \beta}{\rho}}\right) .
$$

Since $1-\eta \omega^{-\frac{\beta}{\rho}}<1$ and $\omega^{-\frac{2 \beta}{\rho}}<1$, a sufficient condition is

$$
\left(\omega^{\frac{\beta}{\rho}}-\omega^{-\frac{\beta}{\rho}}\right)^{2}<\left(\omega^{\frac{\beta}{\rho}}+\omega^{-\frac{\beta}{\rho}}-2 \eta\right)^{2} \Longleftrightarrow 0<\omega^{\frac{\beta}{\rho}}-\eta .
$$

Consider now the $\frac{\lambda}{1-\lambda}\left(\frac{\partial \omega}{\partial \lambda} \frac{\lambda}{\omega}\right)^{-1}$ locus. The trade balance condition (in $\lambda$ notation) implies

$$
T B[\lambda, \omega] \equiv \frac{\eta \omega^{\frac{\beta}{\rho}}-1}{\eta-\omega^{\frac{\beta}{\rho}}} \omega^{-\frac{\beta-\rho}{\rho}}-\frac{1-\lambda}{\lambda}=0 .
$$

By the implicit function theorem, we have

$$
\frac{\partial \omega}{\partial \lambda}=-\frac{\partial T B}{\partial \lambda} / \frac{\partial T B}{\partial \omega},
$$


where

$$
\frac{\partial T B}{\partial \lambda}=\frac{1}{\lambda^{2}}
$$

and

$$
\frac{\partial T B}{\partial \omega}=-\omega^{-\frac{\beta}{\rho}}\left(\frac{\beta}{\rho} \frac{1-\eta^{2}}{\left(\eta-\omega^{\frac{\beta}{\rho}}\right)^{2}} \omega^{\frac{\beta}{\rho}}+\frac{\beta-\rho}{\rho} \frac{\eta \omega^{\frac{\beta}{\rho}}-1}{\eta-\omega^{\frac{\beta}{\rho}}}\right)<0 .
$$

Then,

$$
\frac{\lambda}{1-\lambda}\left(\frac{\partial \omega}{\partial \lambda} \frac{\lambda}{\omega}\right)^{-1}=-\lambda \frac{\lambda}{1-\lambda} \omega^{-\frac{\beta-\rho}{\rho}}\left(\frac{\beta}{\rho} \frac{1-\eta^{2}}{\left(\eta-\omega^{\frac{\beta}{\rho}}\right)^{2}} \omega^{\frac{\beta}{\rho}}+\frac{\beta-\rho}{\rho} \frac{\eta \omega^{\frac{\beta}{\rho}}-1}{\eta-\omega^{\frac{\beta}{\rho}}}\right) .
$$

Using the trade balance condition to substitute out $\lambda /(1-\lambda)$ on the right hand side, we obtain

$$
\begin{aligned}
\frac{\lambda}{1-\lambda}\left(\frac{\partial \omega}{\partial \lambda} \frac{\lambda}{\omega}\right)^{-1} & =-\lambda\left(\frac{\beta}{\rho} \frac{1-\eta^{2}}{\left(\eta-\omega^{\frac{\beta}{\rho}}\right)\left(\eta \omega^{\frac{\beta}{\rho}}-1\right)} \omega^{\frac{\beta}{\rho}}+\frac{\beta-\rho}{\rho}\right) \\
& =\lambda\left(\frac{\beta}{\rho} \frac{1-\eta^{2}}{\eta^{2}+1-\eta\left(\omega^{\frac{\beta}{\rho}}+\omega^{-\frac{\beta}{\rho}}\right)}+\frac{\beta-\rho}{\rho}\right) .
\end{aligned}
$$

Consider the term $\left(\omega^{\frac{\beta}{\rho}}+\omega^{-\frac{\beta}{\rho}}\right)$. Taking the derivative, we obtain

$$
\frac{\partial\left(\omega^{\frac{\beta}{\rho}}+\omega^{-\frac{\beta}{\rho}}\right)}{\partial \omega}=\frac{\beta}{\rho}\left(\omega^{\frac{\beta}{\rho}}-\omega^{-\frac{\beta}{\rho}}\right) \omega^{-1}>0 .
$$

As $\omega$ rises if $\lambda$ goes up, the term $\frac{\lambda}{1-\lambda}\left(\frac{\partial \omega}{\partial \lambda} \frac{\lambda}{\omega}\right)^{-1}$ is increasing in $\lambda$.

To sum up: the downward-sloping $\frac{\lambda}{1-\lambda}\left(\frac{\partial \omega}{\partial \lambda} \frac{\lambda}{\omega}\right)^{-1}$ locus and the upward-sloping $\frac{\lambda}{1-\lambda}\left(\frac{\partial \omega}{\partial \lambda} \frac{\lambda}{\omega}\right)^{-1}$ determine an unique $\lambda^{*}$ such that the firm share $\phi$ increases over-proportionally in $\lambda$ for $\lambda \in\left(0.5, \lambda^{*}\right)$ and less than proportionally for $\lambda \in\left(\lambda^{*}, \bar{\lambda}\right)$. 
Characterization of the threshold degree of external economies of scale $\bar{\mu}$. Recall that

$$
\mathrm{TFP}=N^{\frac{\mu}{\sigma-1}} \tilde{\varphi}
$$

where the relative mass of available inputs $N$ is increasing in relative country size $\mathrm{L}$ and relative average productivity $\tilde{\varphi}$ is decreasing in $L$. We are interested in the degrees of external economies of scale $\mu(L)$ required to (i) have TFP invariant of relative country size.

The elasticity of TFP in relative country size is given by

$$
\begin{aligned}
\frac{d \ln \text { TFP }}{d \ln L} & =\frac{\mu}{\sigma-1} \frac{d \ln N}{d \ln L}+\frac{d \ln \tilde{\varphi}}{d \ln L} \\
& =\frac{\mu}{\sigma-1} \frac{d \ln N}{d \ln L}-\frac{1}{\sigma-1} \frac{d \ln N}{d \ln L}+\frac{1}{\sigma-1}-\frac{1}{\beta} \frac{d \ln \chi}{d \ln L} \\
& =\frac{\mu-1}{\sigma-1} \frac{d \ln N}{d \ln L}+\frac{1}{\sigma-1}-\frac{1}{\beta} \frac{d \ln \chi}{d \ln L} .
\end{aligned}
$$

Setting this expression equal to 0 and solving for $\mu$, we obtain the threshold degree of external economies of scale

$$
\bar{\mu}=\frac{\frac{d \ln N}{d \ln L}+\frac{\sigma-1}{\beta} \frac{d \ln \chi}{d \ln L}-1}{\frac{d \ln N}{d \ln L}}
$$

Recall that the relative mass of available inputs is given by

$$
N=L \times \chi \times \frac{\frac{f^{x}}{f^{d}}+\eta \omega^{\frac{\beta}{\rho}} L^{-1}}{\frac{f^{x}}{f^{d}}+\eta \omega^{-\frac{\beta}{\rho}} L} .
$$

Totally differentiating this expression, we obtain

$$
\begin{aligned}
\frac{d \ln N}{d \ln L} & =1+\frac{d \ln \chi}{d \ln L}+\frac{\eta \omega^{\frac{\beta}{\rho}} L^{-1}}{\frac{f^{x}}{f^{d}}+\eta \omega^{\frac{\beta}{\rho}} L^{-1}}\left(\frac{\beta}{\rho} \frac{d \ln \omega}{d \ln L}-1\right)-\frac{\eta \omega^{-\frac{\beta}{\rho}} L}{\frac{f^{x}}{f^{d}}+\eta \omega^{-\frac{\beta}{\rho}} L}\left(-\frac{\beta}{\rho} \frac{d \ln \omega}{d \ln L}+1\right) \\
& =1+\frac{d \ln \chi}{d \ln L}+\left(\frac{\beta}{\rho} \frac{d \ln \omega}{d \ln L}-1\right)\left(\frac{\eta \omega^{\frac{\beta}{\rho}} L^{-1}}{\frac{f^{x}}{f^{d}}+\eta \omega^{\frac{\beta}{\rho}} L^{-1}}+\frac{\eta \omega^{-\frac{\beta}{\rho}} L}{\frac{f^{x}}{f^{d}}+\eta \omega^{-\frac{\beta}{\rho}} L}\right) .
\end{aligned}
$$

Moreover, the ex post profitability curve reads

$$
\chi=L \omega^{-\frac{2 \beta-\rho}{\rho}} .
$$


Totally differentiating this expression, we have

$$
\frac{d \ln \chi}{d \ln L}=1-\frac{2 \beta-\rho}{\rho} \frac{d \ln \omega}{d \ln L}
$$

Hence, $\frac{\sigma-1}{\beta} \frac{d \ln \chi}{d \ln L}-1<0$, such that for a non-positive constant $c$ the threshold $\mu<1$.

Now consider the neighborhood of a symmetric equilibrium. The change in the mass of available varieties is given by

$$
\frac{d \ln N}{d \ln L}=1+\frac{d \ln \chi}{d \ln L}+\frac{2 \eta\left(\frac{\beta}{\rho} \frac{d \ln \omega}{d \ln L}-1\right)}{\frac{f^{x}}{f^{d}}+\eta}
$$

The change in the relative wage is

$$
\begin{aligned}
\frac{d \ln \omega}{d \ln L} & =\rho\left(\beta \frac{2 \omega^{\frac{\beta}{\rho}}-\eta\left(\omega^{\frac{2 \beta}{\rho}}+1\right)}{\left(\omega^{\frac{\beta}{\rho}}-\eta\right)\left(1-\eta \omega^{\frac{\beta}{\rho}}\right)}-\rho\right)^{-1} \\
& =\rho\left(\beta \frac{2-2 \eta}{(1-\eta)(1-\eta)}-\rho\right)^{-1} \\
& =\frac{\rho(1-\eta)}{2 \beta-\rho(1-\eta)}
\end{aligned}
$$

The change in the relative entry probability is

$$
\begin{aligned}
\frac{d \ln \chi}{d \ln L} & =1-\frac{2 \beta-\rho}{\rho} \frac{d \ln \omega}{d \ln L} \\
& =1-\frac{2 \beta-\rho}{\rho} \frac{\rho(1-\eta)}{2 \beta-\rho(1-\eta)} \\
& =1-\frac{(2 \beta-\rho)(1-\eta)}{2 \beta-\rho(1-\eta)} \\
& =\frac{2 \beta-\rho(1-\eta)-(2 \beta-\rho)(1-\eta)}{2 \beta-\rho(1-\eta)} \\
& =\frac{2 \beta-2 \beta(1-\eta)}{2 \beta-\rho(1-\eta)} \\
& =\frac{2 \beta \eta}{2 \beta-\rho(1-\eta)} .
\end{aligned}
$$


Hence, we have

$$
\begin{aligned}
\frac{d \ln N}{d \ln L} & =1+\frac{2 \beta \eta}{2 \beta-\rho(1-\eta)}+\frac{2 \eta\left(\frac{\beta}{\rho} \frac{\rho(1-\eta)}{2 \beta-\rho(1-\eta)}-1\right)}{\frac{f^{x}}{f^{d}}+\eta} \\
& =1+\frac{2 \beta \eta}{2 \beta-\rho(1-\eta)}+\frac{2 \eta\left(\frac{\beta(1-\eta)}{2 \beta-\rho(1-\eta)}-1\right)}{\frac{f^{x}}{f^{d}}+\eta} \\
& =1+\frac{2 \beta \eta}{2 \beta-\rho(1-\eta)}+\frac{2 \eta \frac{\beta(1-\eta)-2 \beta+\rho(1-\eta)}{2 \beta-\rho(1-\eta)}}{\frac{f^{x}}{f^{d}}+\eta} \\
& =1+\frac{2 \beta \eta}{2 \beta-\rho(1-\eta)}+2 \eta \frac{(\beta+\rho)(1-\eta)-2 \beta}{\left(\frac{f^{x}}{f^{d}}+\eta\right)[2 \beta-\rho(1-\eta)]} \\
& =\frac{2 \beta-\rho(1-\eta)+2 \beta \eta}{2 \beta-\rho(1-\eta)}+2 \eta \frac{(\beta+\rho)(1-\eta)-2 \beta}{\left(\frac{f^{x}}{f^{d}}+\eta\right)[2 \beta-\rho(1-\eta)]} \\
= & \frac{2 \beta(1+\eta)-\rho(1-\eta)}{2 \beta-\rho(1-\eta)}+2 \eta \frac{(\beta+\rho)(1-\eta)-2 \beta}{\left(\frac{f^{x}}{f^{d}}+\eta\right)[2 \beta-\rho(1-\eta)]} \\
= & \frac{[2 \beta(1+\eta)-\rho(1-\eta)]\left(\frac{f^{x}}{f^{d}}+\eta\right)}{\left(\frac{f^{x}}{f^{d}}+\eta\right)[2 \beta-\rho(1-\eta)]}+2 \eta \frac{(\beta+\rho)(1-\eta)-2 \beta}{\left(\frac{f^{x}}{f^{d}}+\eta\right)[2 \beta-\rho(1-\eta)]}
\end{aligned} .
$$

\section{Moreover,}

$$
\begin{aligned}
\frac{d \ln N}{d \ln L}+\frac{\sigma-1}{\beta} \frac{d \ln \chi}{d \ln L}-1 & =1+\frac{2 \beta \eta}{2 \beta-\rho(1-\eta)}+2 \eta \frac{(\beta+\rho)(1-\eta)-2 \beta}{\left(\frac{f^{x}}{f^{d}}+\eta\right)[2 \beta-\rho(1-\eta)]}+\frac{\sigma-1}{\beta} \frac{2 \beta \eta}{2 \beta-\rho(1-\eta)}-1 \\
& =\frac{2 \beta \eta\left(1+\frac{\sigma-1}{\beta}\right)}{2 \beta-\rho(1-\eta)}+2 \eta \frac{(\beta+\rho)(1-\eta)-2 \beta}{\left(\frac{f^{x}}{f^{d}}+\eta\right)[2 \beta-\rho(1-\eta)]} \\
& =\frac{2 \eta(\beta+\sigma-1)}{2 \beta-\rho(1-\eta)}+2 \eta \frac{(\beta+\rho)(1-\eta)-2 \beta}{\left(\frac{f^{x}}{f^{d}}+\eta\right)[2 \beta-\rho(1-\eta)]} \\
& =\frac{2 \eta(\beta+\sigma-1)\left(\frac{f^{x}}{f^{d}}+\eta\right)}{\left(\frac{f^{x}}{f^{d}}+\eta\right)[2 \beta-\rho(1-\eta)]}+2 \eta \frac{(\beta+\rho)(1-\eta)-2 \beta}{\left(\frac{f^{x}}{f^{d}}+\eta\right)[2 \beta-\rho(1-\eta)]}
\end{aligned}
$$

Using the above expressions, we obtain a closed-form solution for $\bar{\mu}$ in the neighborhood of 
a symmetric equilibrium:

$$
\begin{aligned}
\bar{\mu} & =\frac{2 \eta(\beta+\sigma-1)\left(\frac{f^{x}}{f^{d}}+\eta\right)+2 \eta(\beta+\rho)(1-\eta)-4 \beta \eta}{[2 \beta(1+\eta)-\rho(1-\eta)]\left(\frac{f^{x}}{f^{d}}+\eta\right)+2 \eta(\beta+\rho)(1-\eta)-4 \beta \eta} \\
& =2 \eta \frac{(\beta+\sigma-1)\left(\frac{f^{x}}{f^{d}}+\eta\right)+(\beta+\rho)(1-\eta)-2 \beta}{[2 \beta(1+\eta)-\rho(1-\eta)]\left(\frac{f^{x}}{f^{d}}+\eta\right)+2 \eta(\beta+\rho)(1-\eta)-4 \beta \eta} .
\end{aligned}
$$

\section{D.2 Unconventional sorting}

It is a well documented stylized fact that only the most productive firms engage in exporting; see Bernard, Jensen, Redding, and Schott (2007). With symmetric countries, this sorting pattern obtains if one assumes that trade costs are sufficiently large: $\tau^{\sigma-1} f^{x}>f^{d}$; see Melitz (2003).

With asymmetric countries, the sorting pattern can reverse in the smaller country. Firms with intermediate productivity levels may make positive profits from exporting to the large country, while they would make negative profits on their small domestic market. This "unconventional" sorting requires to adapt the definition of becoming active, which has implications for the relative entry probability $\chi$ and the mass of active firms.

Conventional sorting occurs as long as

$$
\varphi_{F H}^{*}>\varphi_{F F}^{*} \Leftrightarrow \tau^{\sigma-1} \frac{f^{x}}{f^{d}} \frac{E_{F} N_{F}^{\mu-1} P_{F}^{\sigma-1}}{E_{H} N_{H}^{\mu-1} P_{H}^{\sigma-1}}>1 .
$$

Using the balanced trade condition, which still holds under unconventional sorting, to substitute out $\varphi_{F H}^{*}$, we obtain

$$
\varphi_{H F}^{*}(L \omega)^{-\frac{1}{\beta}}>\varphi_{F F}^{*} \Leftrightarrow \tau^{\sigma-1} \frac{f^{x}}{f^{d}} \omega^{\frac{1}{\rho}}(L \omega)^{-\frac{1}{\beta}}>1 .
$$

Conventional sorting cutoff. Let $\bar{L}$ denote the relative size cutoff up to which Foreign firms with intermediate productivity levels serve the domestic, but not the export market. It is 
implicitly defined by setting $\varphi_{F F}^{*}=\varphi_{F H}^{*}$ :

$$
\varphi_{F F}^{*}=\varphi_{F H}^{*} \Leftrightarrow \varphi_{H F}^{*}(L \omega)^{-\frac{1}{\beta}}=\varphi_{F F}^{*} \Leftrightarrow 1=\tau^{-1} \bar{\omega}^{-\frac{\beta-\rho}{\rho \beta}} \bar{L}^{\frac{1}{\beta}}\left(\frac{f^{x}}{f^{d}}\right)^{-\frac{1}{\sigma-1}}
$$

Setting the ex ante and the ex post profitability curves equal, we the find that the relative wage $\bar{\omega}$ is implicitly defined as:

$$
\bar{L} \bar{\omega}^{-\frac{2 \beta-\rho}{\rho}}=\frac{1-\eta \bar{\omega}^{-\frac{\beta}{\rho}}}{1-\eta \bar{\omega}^{\frac{\beta}{\rho}}} \Leftrightarrow \bar{L}=\bar{\omega}^{\frac{\beta-\rho}{\rho}} \frac{\bar{\omega}^{\frac{\beta}{\rho}}-\eta}{1-\eta \bar{\omega}^{\frac{\beta}{\rho}}} .
$$

Using this expression to substitute out $\bar{L}$ from the above condition, we obtain

$$
\begin{aligned}
1 & =\tau^{-1} \bar{\omega}^{-\frac{\beta-\rho}{\rho \beta}} \bar{\omega}^{\frac{\beta-\rho}{\beta \rho}}\left(\frac{\bar{\omega}^{\frac{\beta}{\rho}}-\eta}{1-\eta \bar{\omega}^{\frac{\beta}{\rho}}}\right)^{\frac{1}{\beta}}\left(\frac{f^{x}}{f^{d}}\right)^{-\frac{1}{\sigma-1}} \Leftrightarrow \frac{1-\eta \bar{\omega}^{\frac{\beta}{\rho}}}{\bar{\omega}^{\frac{\beta}{\rho}}-\eta}=\tau^{-\beta}\left(\frac{f^{x}}{f^{d}}\right)^{1-\frac{\beta}{\sigma-1}-1} \Leftrightarrow \\
\frac{f^{x}}{f^{d}}\left(1-\eta \bar{\omega}^{\frac{\beta}{\rho}}\right) & =\eta\left(\bar{\omega}^{\frac{\beta}{\rho}}-\eta\right) \Leftrightarrow \frac{f^{x}}{f^{d}}-\eta \frac{f^{x}}{f^{d}} \bar{\omega}^{\frac{\beta}{\rho}}=\eta \bar{\omega}^{\frac{\beta}{\rho}}-\eta^{2} \Leftrightarrow \eta \bar{\omega}^{\frac{\beta}{\rho}}\left(\frac{f^{x}}{f^{d}}+1\right)=\frac{f^{x}}{f^{d}}+\eta^{2} \Leftrightarrow \\
\bar{\omega}^{\frac{\beta}{\rho}} & =\frac{\frac{f^{x}}{f^{d}} \eta^{-1}+\eta}{\frac{f^{x}}{f^{d}}+1},
\end{aligned}
$$

which solves for $\bar{\omega}$ as a function of exogenous parameters. Substituting back this solution for $\bar{\omega}$, we can compute $\bar{L}$ from the expression above as a function of exogenous parameters.

Equilibrium. The relative wage is implicitly determined by

$$
L \omega^{-\frac{2 \beta-\rho}{\rho}}=\frac{1-\eta \omega^{-\frac{\beta}{\rho}}}{1-\eta \omega^{\frac{\beta}{\rho}}}
$$

which is the same equilibrium condition as in the case of conventional sorting. The reason is the following. Expected profits in Foreign are given by

$$
\bar{\pi}_{F}=\int_{\varphi_{F F}^{*}} \pi_{F F}[\varphi] \frac{d G[\varphi]}{1-G\left[\varphi_{F H}^{*}\right]}+\int_{\varphi_{F H}^{*}} \pi_{F H}[\varphi] \frac{d G[\varphi]}{1-G\left[\varphi_{F H}^{*}\right]},
$$


where we condition the ex ante productivity distribution on the probability of successful entry, $1-G\left[\varphi_{F H}^{*}\right]$. Using the Pareto assumption and the zero cutoff entry conditions, we obtain

$$
\bar{\pi}_{F}=(\theta-1)\left(\left(\frac{\varphi_{F F}^{*}}{\varphi_{F H}^{*}}\right)^{-\beta} f^{d}+f^{x}\right) w_{F} .
$$

The free entry condition reads

$$
\left(1-G\left[\varphi_{F H}^{*}\right]\right) \bar{\pi}_{F}=w_{F} f^{e}
$$

Using the expression for expected profits from above and the employing Pareto, we obtain

$$
(\theta-1)\left(\left(\varphi_{F F}^{*}\right)^{-\beta} f^{d}+\left(\varphi_{F H}^{*}\right)^{-\beta} f^{x}\right)=f^{e}
$$

which is exactly the same equilibrium condition as in the conventional sorting case. Hence, we can solve for relative domestic entry cutoffs in the same way as under conventional sorting, the only difference being that relative domestic cutoffs no longer represent the relative probability of successful entry.

The relative entry probability $\chi$ is now defined as

$$
\chi \equiv \frac{1-G\left[\varphi_{H H}^{*}\right]}{1-G\left[\varphi_{F H}^{*}\right]}=\left(\frac{\varphi_{F H}^{*}}{\varphi_{H H}^{*}}\right)^{\beta} .
$$

The two zero cutoff profit conditions from targeting Home imply

$$
\chi=\tau^{\beta}\left(\frac{f^{x}}{f^{d}}\right)^{\frac{\beta}{\sigma-1}} \omega^{-\frac{\beta}{\rho}}=\frac{f^{x}}{f^{d}} \eta^{-1} \omega^{-\frac{\beta}{\rho}} .
$$

Given the equilibrium wage from above, this expression solves for $\chi$. Notice that

$$
\eta \omega^{\frac{\beta}{\rho}}<1 \Leftrightarrow \eta^{-} \omega^{-\frac{\beta}{\rho}}>1
$$

Hence, a sufficient condition for $\chi>1$ (Home Market Effect) is $f^{x}>f^{d}$. 


\section{D.3 Melitz (2003) with outside sector}

In this appendix, we derive equilibrium in the presence of an outside sector. We show that the economy exhibits a home market effect. As in Helpman and Krugman (1985), the home market effect is linear in $\lambda$. Moreover, we discuss welfare implications for the standard CES case.

Basic environment. The model is augmented by a homogeneous good produced under constant returns to scale and perfect competition. Utility takes the Cobb-Douglas form, where $\mu$ denotes the share of expenditure spent on differentiated varieties. The outside good is freely tradable. Hence, wages are equalized and henceforth normalized to unity. Welfare per worker is given by the inverse of the aggregate price index. Using $P_{i}$ to denote the price index of the differentiated goods sector and defining $\tilde{\mu} \equiv(1-\mu)^{1-\mu} \mu^{\mu}$, we can write welfare per worker as

$$
W_{i}=\tilde{\mu} / P_{i}^{\mu}
$$

Equilibrium. The free entry conditions are unaffected. In the zero cutoff profit conditions, however, wages drop. In relative form, the zero cutoff profit condition becomes

$$
\varphi_{j i}^{*}=\tau\left(\frac{f^{x}}{f^{d}}\right)^{\frac{1}{\sigma-1}} \varphi_{i i}^{*} .
$$

Hence, we can substitute out export cutoffs from the free entry conditions. We obtain two equations in two unknowns, which can be used to solve for the domestic entry cutoffs as

$$
\left(\varphi_{i i}^{*}\right)^{\beta}=(\theta-1) \frac{\left(f^{d}\right)^{\beta}}{f^{e}}(1+\eta)
$$

It is important to note that the cutoff productivity levels do not depend on country size. Hence, they are symmetric across countries irrespectively of the country size distribution. 
Labor market clearing implies

$$
\xi_{i} L_{i}=M_{i}^{e} f^{e}+M_{i} \sum_{j} m_{i j} f_{i j}+M_{i} \sum_{j} \int_{\varphi_{i j}^{*}} \frac{\tau_{i j} q_{i j}[\varphi]}{\varphi} \frac{d G[\varphi]}{1-G\left[\varphi_{i j}^{*}\right]}=M_{i} \theta \sum_{j} m_{i j} f_{i j}
$$

where $\xi_{i}$ denotes the fraction of workers employed in the differentiated good sector.

Using the free entry condition, we obtain

$$
M_{i}=\frac{\xi_{i} L_{i}}{\bar{r}_{i}}
$$

where $\bar{r}_{i} \equiv \sum_{j} \bar{r}_{i j}=\frac{\beta f^{e}}{\rho}\left(\varphi_{i i}^{*}\right)^{\beta}$. Expected revenues $\bar{r}_{i j}$ are given by

$$
\bar{r}_{i j}=\int_{\varphi_{i j}^{*}} r_{i j}[\varphi] \frac{d G[\varphi]}{1-G\left[\varphi_{i j}^{*}\right]}=\theta \sigma m_{i j} f_{i j}
$$

Due to the symmetry of the cutoffs, we henceforth suppress the subscripts of the revenue terms. Note that $\bar{r}^{x} / \bar{r}^{d}$ reduces to $\eta$.

Balanced trade is given by

$$
M_{i} \bar{r}^{x}=M_{j} \bar{r}^{x}+(1-\mu) L_{i}-\left(1-\xi_{i}\right) L_{i}
$$

where the term on the left hand side represents country $i$ 's exports of the differentiated good. The first term on the right hand side represents $i$ 's imports of the differentiated good. The remaining terms reflect $i$ 's imports of the homogeneous good (spending on the homogeneous good minus value of domestic homogeneous good production).

Substituting out $M_{i}$ and $\bar{r}_{i j}$ from balanced trade and using $\bar{r}_{i}=\bar{r}_{j}$, we obtain

$$
\xi_{i}=\mu \frac{\bar{r}}{\bar{r}^{d}}-\xi_{j} \frac{L_{j}}{L_{i}} \frac{\bar{r}^{x}}{\bar{r}^{d}}, i \in\{H, F\}
$$

Solving the system of two equations in $\xi_{i}$ and $\xi_{j}$, we obtain

$$
\xi_{i}=\mu \frac{\frac{\bar{r}}{\bar{r} d}-\frac{L_{j}}{L_{i}} \frac{\bar{r}^{x}}{\bar{r}^{x}} \frac{\bar{r}}{\bar{r}^{d}}}{1-\left(\frac{\bar{r}^{x}}{\bar{r}^{d}}\right)^{2}}, i \in\{H, F\}
$$


Moreover, we have

$$
\xi_{H}>\xi_{F} \Leftrightarrow \lambda>1 / 2
$$

Hence, the smaller country is a net exporter of the homogeneous good.

Home market effect. The labor clearing conditions implies that Home's share of firms active in the differentiated good sector can be written as

$$
\phi=\frac{1}{\lambda+(1-\lambda) \frac{\xi_{F}}{\xi_{H}}} \lambda .
$$

Note that entry cutoffs have dropped due to their symmetry. We have seen above that $\xi_{H}>$ $\xi_{F}$, which constitutes a weak home market effect.

We can rewrite $\phi$ as

$$
\phi=\frac{1}{1+\frac{1-\lambda}{\lambda} \frac{\bar{r}-\frac{\lambda}{1-\lambda} \bar{r}^{x}}{\bar{r}-\frac{1-\lambda}{\lambda}}}=\frac{\lambda \bar{r}-(1-\lambda) \bar{r}^{x}}{\bar{r}^{d}}
$$

with

$$
\frac{\partial \phi}{\partial \lambda}=1+2 \frac{\bar{r}^{x}}{\bar{r}^{d}}=1+2 \eta>1
$$

The following observations stand out. First, the HME is linear in $\lambda$. Second, the increase of the firm share in the endowment share is larger than one. Third, the HME is magnified by a reduction in trade barriers (HMME).

Welfare. Using the domestic zero cutoff profit condition, we can rewrite welfare per worker as

$$
W_{i}=\tilde{\mu}\left(\frac{\beta L_{i}}{\sigma f^{d}}\right)^{\frac{\mu}{\sigma-1}}\left(\rho \varphi_{i i}^{*}\right)^{\mu}
$$

where $\tilde{\mu} \equiv(1-\mu)^{1-\mu} \mu^{\mu}$ is a constant. The following observations stand out. First, a shock on the relative country size $\lambda$ affects both countries symmetrically. The reason that domestic entry cutoffs are independent of the country size distribution. Second, an increase in freeness of trade rises the domestic entry cutoff and therefore increases welfare per worker in both countries. Third, an increase in the freeness of trade leaves relative welfare per worker unaffected. 